\title{
Progress in diagnostic testing and management of idiopathic ventricular fibrillation
}

Lennart J. Blom 




\title{
Progress in diagnostic testing and management of idiopathic ventricular fibrillation
}

\author{
Vooruitgang in diagnostiek en behandeling van idiopathisch \\ ventrikelfibrilleren \\ (met een samenvatting in het Nederlands)
}

\begin{abstract}
PROEFSCHRIFT
ter verkrijging van de graad van doctor aan de

Universiteit Utrecht

op gezag van de

rector magnificus, prof.dr. H.R.B.M. Kummeling, ingevolge het besluit van het college voor promoties

in het openbaar te verdedigen op
\end{abstract}

dinsdag 6 oktober 2020 des ochtends te 11.00 uur

door

\section{Lennart Johannes Blom}

geboren op 27 november 1990

te Bergen op Zoom 


\section{Promotor:}

Prof.dr. P.A.F.M. Doevendans

\section{Copromotor:}

Dr. R.J. Hassink

Financial support by the Dutch Heart Foundation for the publication of this thesis is gratefully acknowledged. 
Cover illustration: Lennart Blom

(C) 2020 Lennart Blom

All Rights Reserved.

ISBN: 978-90-393-7252-4 


\section{CONTENTS}

Chapter 1 Introduction

Chapter 2 Life-long tailoring of diagnosis and management of patients with idiopathic ventricular fibrillation - future perspectives in research.

Chapter 3 Sudden cardiac death in the young: Post-mortem investigation and cardiogenetic evaluation of victims and their relatives.

Chapter 4 Incidence and predictors of implantable cardioverterdefibrillator therapy and its complications in idiopathic ventricular fibrillation patients.

Chapter 5 Familial cascade screening in idiopathic ventricular fibrillation.

Chapter 6 Novel use of repolarization parameters in electrocardiographic imaging to uncover arrhythmogenic substrate.

Chapter 7 Automatic triage of 12-lead electrocardiograms using deep convolutional neural networks.

Chapter 8 Late evolution of arrhythmogenic cardiomyopathy in patients with initial presentation as idiopathic ventricular fibrillation.

Chapter 9 Discussion

Appendices

Nederlandse Samenvatting

Dankwoord

Curriculum Vitae 

Chapter

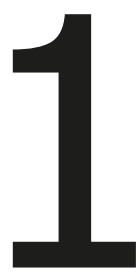

Introduction 
Electrical excitation and repolarization of the heart is a well-balanced and regulated process. ${ }^{1}$ Though the majority of myocardial cells is excitable, a specialized set of cells known as the conduction system ensures structured propagation of action potentials over the myocardium leading to contraction of the heart and cardiac output. ${ }^{2}$ Local cellular properties and conditions facilitate an ordered and timely return to their polarized state. When this process is disturbed, the absence of a normal heart rhythm or the emergence of cardiac arrhythmias can lead to loss of circulation and death. When this occurs in a sudden and unexpected manner, it is referred to as sudden cardiac arrest (SCA). ${ }^{3}$

Ventricular fibrillation is the underlying arrhythmia leading to SCA in most young cardiac arrest victims. ${ }^{4}$ Mechanisms of ventricular fibrillation are classically represented in Coumel's triangle of arrhythmogenesis: the arrhythmogenic substrate, the trigger factor and the modulation factors. To facilitate ventricular fibrillation, a substrate, trigger and the right modulating factors should all be present. A substrate may be scar tissue, remodelled myocardium or ion-channel dysfunction. Triggers are predominantly ventricular extrasystoles. Modulating factors include the autonomous nervous system, electrolyte disturbances or ischemia. Since demonstrating the presence of substrate is the least challenging, diagnostics tend to concentrate on this.

Understanding of the underlying pathology that has led to sudden cardiac arrest is of utmost importance. ${ }^{5,6}$ In survivors, understanding of the underlying cause is required to provide adequate treatment and prevent recurrence of ventricular arrhythmias and subsequent sudden cardiac death after the initial event. In those who do not survive, learning the cause of death has important consequences for management of their relatives at risk. ${ }^{7,8}$

Numerous diagnostic tools are available to investigate the underlying cause of SCA. ${ }^{6}$ First of all, the most common cause of SCA should be excluded: Coronary artery disease. Diagnosis of coronary artery disease is relatively well established and can be done using coronary angiography 
or computed tomography (CT) / magnetic resonance imaging (MRI) in younger patients. Second, structural heart disease should be excluded. Non-invasive imaging techniques, such as CT/MRI and echocardiography assess the function, dimensions and tissue of the heart for signs of structural disease. Third, we assess the electrical properties of the heart to exclude (primary) electrical disease. The key diagnostic tests herein are the electrocardiogram, Holter monitoring, exercise tests and provocation testing.

Genetic testing has become an integral part of the management of SCA victims, predominantly in young victims, those with a family history of sudden cardiac death or those who have family members who could be at risk. ${ }^{6,9,10}$ In a large part of cardiomyopathy and primary electrical syndrome cohorts an associated causative mutation was found. Discovery of such a mutation in a cardiac arrest victim will lead to diagnosis in most cases. However, genetic variants of uncertain significance and variable penetrance complicate the certainty of diagnosis in SCA victims and the management of their family members.

When no underlying cause has been identified in a cardiac arrest survivor after this extensive work-up, he is diagnosed with idiopathic ventricular fibrillation (IVF). ${ }^{11}$ Due to the rarity and unknown pathology of the disease, management of these patients is challenging. ${ }^{12}$

\section{Challenges in idiopathic ventricular fibrillation}

The absence of an underlying cause is unsatisfactory for both patients, family members and physicians. Although there is increasing interest in IVF, several major challenges remain. First, a comprehensive work-up with all necessary diagnostic tests is not always performed in the real-world population of IVF patients and SCD victims. Second, novel diagnostic tests have not yet been studied in IVF patients and could be of added value in the search for underlying disease. Third, management of IVF patients and family members is not well specified. Which groups have a higher risk of appropriate and inappropriate therapy after ICD implantation? And how useful is the phenotypic screening of family members when no underlying 
disease has been found in the proband? The aim of this thesis was to study current and novel diagnostics for idiopathic ventricular fibrillation and to evaluate current management of idiopathic ventricular fibrillation and family members.

\section{Outline of this thesis}

In this thesis, "Progress in diagnostic testing and management of idiopathic ventricular fibrillation", we present and discuss the current insight in and future possibilities of diagnostic testing and management of idiopathic ventricular fibrillation patients. In Chapter 2, we review the current clinical management of idiopathic ventricular fibrillation with a focus on our research initiatives and population in The Netherlands and the use of novel imaging techniques.

The evaluation of young sudden cardiac death victims and their family members is then discussed in Chapter 3. The investigations in this group and consequences for management differ from SCA survivors and require a different clinical approach. We review the value of diagnostic testing and provide a flowchart for clinical management. In Chapter $\mathbf{4}$ we describe the management of idiopathic VF patients and focus on the implantable cardioverter-defibrillator (ICD) therapy and its complications. This chapter shows the recurrence of ventricular arrhythmia's is common in these patients, justifying ICD implantation, while both inappropriate therapy and complications occur frequently. Chapter $\mathbf{5}$ deals with family cascade screening in idiopathic ventricular fibrillation. Results from an international collaboration including multiple IVF family member cohorts show that the yield of family screening in families of well characterized IVF patients is low and might be unnecessary.

In Chapter $\mathbf{6}$ we explore the potential of novel diagnostic techniques. In this study we focus on the mapping of arrhythmogenic substrate using electrocardiographic imaging (ECGI) and the value of additional repolarization parameters in this technique. In Chapter $\mathbf{7}$ we look into the possibilities of deep neural networks for analysis of electrocardiographic signals. As they perform well on classification tasks and their ability to detect subtle features improves, they may well be used for early detection 
of disease in the near future.

Finally, in Chapter $\mathbf{8}$ through a number of clinical cases we illustrate the progress that has already been made in diagnosis and management of IVF and the importance of extensive diagnostic testing and follow-up. 


\section{References}

1. Conrath CE, Opthof T: Ventricular repolarization: An overview of (patho)physiology, sympathetic effects and genetic aspects. Prog Biophys Mol Biol 2006; 92:269-307.

2. Durrer D, van Dam RT, Freud GE, Janse MJ, Meijler FL, Arzbaecher RC: Total excitation of the isolated human heart. Circulation 1970; 41:899912.

3. Zipes DP, Wellens HJJ: Sudden Cardiac Death. Circulation 1998; 98:2334-2351.

4. Ackerman MJ, Atkins DL, Triedman JK: Sudden cardiac death in the young. Circulation 2016; 133:1006-1026.

5. Cheung CC, Krahn AD: The importance of a comprehensive evaluation of survivors of cardiac arrest. Eur Heart J 2018; 39:1988-1991.

6. Visser M, van der Heijden JF, Doevendans PA, Loh P, Wilde AA, Hassink $\mathrm{RJ}$ : Idiopathic Ventricular Fibrillation. Circ Arrhythmia Electrophysiol 2016; 9:e003817.

7. Steinberg C, Padfield GJ, Champagne J, et al.: Cardiac Abnormalities in First-Degree Relatives of Unexplained Cardiac Arrest Victims. Circ Arrhythmia Electrophysiol 2016; 9.

8. Kumar S, Peters S, Thompson T, Morgan N, Maccicoca I, Trainer A, Zentner D, Kalman JM, Winship I, Vohra JK: Familial cardiological and targeted genetic evaluation: low yield in sudden unexplained death and high yield in unexplained cardiac arrest syndromes. Hear Rhythm 2013; 10:1653-1660.

9. Mellor G, Laksman ZWM, Tadros R, et al.: Genetic Testing in the Evaluation of Unexplained Cardiac Arrest: From the CASPER (Cardiac Arrest Survivors with Preserved Ejection Fraction Registry). Circ Cardiovasc Genet 2017; 10:1-8.

10. Leinonen JT, Crotti L, Djupsjöbacka $A$, et al.: The genetics underlying idiopathic ventricular fibrillation: A special role for catecholaminergic polymorphic ventricular tachycardia? Int J Cardiol 2018; 250:139-145.

11. Priori SG, Wilde AA, Horie M, et al.: HRS/EHRA/APHRS Expert Consensus Statement on the Diagnosis and Management of Patients 


\section{INTRODUCTION | 15}

with Inherited Primary Arrhythmia Syndromes. Hear Rhythm 2013; 10:1932-1963.

12. Priori SG, Blomström-Lundqvist C, Mazzanti A, et al.: 2015 ESC Guidelines for the management of patients with ventricular arrhythmias and the prevention of sudden cardiac death. Eur Heart J 2015; 36:2793-2867. 



\section{Chapter}

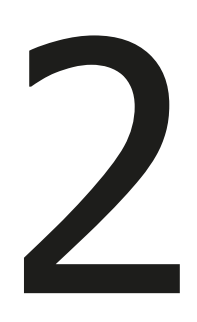

\section{Life-long tailoring of diagnosis and management of patients with idiopathic ventricular fibrillation - future perspectives in research.}

L.J. Blom ${ }^{1}$, P.G.A. Volders' ${ }^{2}$ A.A. Wilde ${ }^{3}$, R.J. Hassink ${ }^{1}$

1 Department of Cardiology, University Medical Center, Utrecht, The Netherlands

2 Department of Cardiology, Cardiovascular Research Institute Maastricht (CARIM),

Maastricht University Medical Center, Maastricht, The Netherlands

3 Department of Clinical and Experimental Cardiology, Heart Centre, Academic Medical Center (AMC), Amsterdam, The Netherlands

Published in Netherlands Heart Journal 2018 


\section{8 | CHAPTER 2}

\section{ABSTRACT}

The diagnosis and management of idiopathic ventricular fibrillation is challenging, as it requires extensive diagnostic testing and offers few curative options due to unknown underlying disease. The resulting population is a heterogeneous group of patients with a largely unknown natural history. Structural patient characterisation, follow-up and innovations in diagnostic testing can improve our understanding of the disease mechanisms of idiopathic ventricular fibrillation, detect underlying disease during follow-up and aid in therapeutic management. Recently, initiatives have been launched in the Netherlands to investigate the role of high-resolution non-invasive electrocardiographic imaging and genetic and familial screening in idiopathic ventricular fibrillation. 


\section{INTRODUCTION}

Idiopathic ventricular fibrillation is a rare cause of sudden cardiac arrest, defined as 'resuscitated cardiac arrest, preferably with documentation of ventricular fibrillation, for which known cardiac, respiratory, metabolic, and toxicological aetiologies have been excluded through clinical evaluation ${ }^{\prime 1}$. In general, patients with idiopathic ventricular fibrillation are young (average age: 38 ) and present with a structurally normal heart. Specific genetic disorders and diseases, such as primary arrhythmia syndromes, should be evaluated in these patients ${ }^{1-3}$. Limited data are available on the natural history of idiopathic ventricular fibrillation, including identification of putative pathogenic mutations, and management of patients with idiopathic ventricular fibrillation and their family members ${ }^{2,4}$.

Idiopathic ventricular fibrillation is a diagnosis of exclusion and therefore patients undergo a broad range of diagnostic tests. We proposed a flowchart to standardise diagnostic testing in patients with idiopathic ventricular fibrillation ${ }^{2}$. Routine testing comprises electrocardiogram (ECG), blood chemistry (cardiac enzymes, electrolytes, and thyroid function), toxicology screening, chest X-ray, echocardiography, exercise testing, Holter or telemetry monitoring, coronary angiography with or without ventriculography, and magnetic resonance imaging. When these tests reveal no abnormalities, provocation tests for Brugada syndrome (ajmaline/flecainide test) and coronary artery spasm (ergonovine/ acetylcholine test) are recommended.

Additional testing, such as endomyocardial biopsy, electrophysiological studies and genetic testing, is under debate, as their diagnostic value in idiopathic ventricular fibrillation is uncertain ${ }^{2}$. The yield of genetic testing in idiopathic ventricular fibrillation is interesting, as it contributed immensely to the detection of primary arrhythmia syndromes. However, a clinical suspicion, based on phenotype, should guide genetic testing. Even with next generation sequencing, which screens large gene panels at once, the yield is minimal and variants of uncertain significance are often detected ${ }^{5}$. Proposed genetic testing entails a basic panel of SCN5A, the most common long QT genes (KCNQ1 and KCNH2), RyR2, and CALM1 


\section{0 |CHAPTER 2}

in patients with exercise-induced ventricular fibrillation. In patients with a negative phenotype, we recommend screening of SCN5A, KCNQ1, and $\mathrm{KCNH}^{2}$. In the central part of the Netherlands and in families originating from the Gouda region, DPP6 screening is recommended as the DPP6 haplotype accounts for a large part of the idiopathic ventricular fibrillation population (over $25 \%$ ) in this area6.

In current practice, patients are diagnosed with idiopathic ventricular fibrillation after limited diagnostic testing ${ }^{7,8}$. Therefore, follow-up and reevaluation of the diagnosis are important aspects of the management of patients with idiopathic ventricular fibrillation. Of patients with idiopathic ventricular fibrillation, 7 to $35 \%$ reveal a different diagnosis during followup due to disease progression or more extensive or more sophisticated diagnostic evaluation ${ }^{9-13}$. Since the underlying disease substrate is often not known, discovery of new disease entities and novel diagnostic techniques can reveal causes not detectable or known during initial evaluation.

\section{A PATIENT INITIALLY DIAGNOSED WITH IDIOPATHIC VENTRICULAR FIBRILLATION}

To support the importance of life-long tailoring of diagnosis and management of patients with idiopathic ventricular fibrillation we describe a case of idiopathic ventricular fibrillation presented to our centre:

A 24-year-old man collapsed twice in one year while playing a football match. The first time, in 1991, he regained consciousness shortly after resuscitation was started, and clinical evaluation was inconclusive. The second time, in 1992, he received 2 external defibrillator shocks because of ventricular fibrillation. After successful resuscitation he was admitted for diagnostic evaluation. Electrocardiography showed a sinus rhythm with intraventricular conduction delay (QRS 140ms), including prolonged terminal activation duration (TAD 70ms), and J-point elevation in the inferior leads (Fig. 1). History, Holter monitoring and exercise test were unremarkable, no ventricular extrasystoles were reported. Family 


\section{LIFE-LONG TAILORING OF DIAGNOSIS AND MANAGEMENT OF IVF| 21}

history was negative for sudden cardiac death. Echocardiogram showed normal cardiac function and normal right and left ventricular dimensions, with a local abnormality under the tricuspid valve. Transoesophageal echocardiogram characterised this as prolapse of a valve leaflet. During electrophysiologic study, a prolonged HV interval of $60-70 \mathrm{~ms}$ was measured. Right ventricular stimulation induced polymorphic ventricular tachycardia starting as monomorphic ventricular tachycardia with left bundle branch block morphology and superior axis. Other diagnostic tests, including coronary angiogram, myocardial biopsy and ergonovine provocation test, were normal. He received an implantable cardioverterdefibrillator (ICD) and was discharged. In the absence of an obvious aetiology, he was diagnosed with idiopathic ventricular fibrillation. 

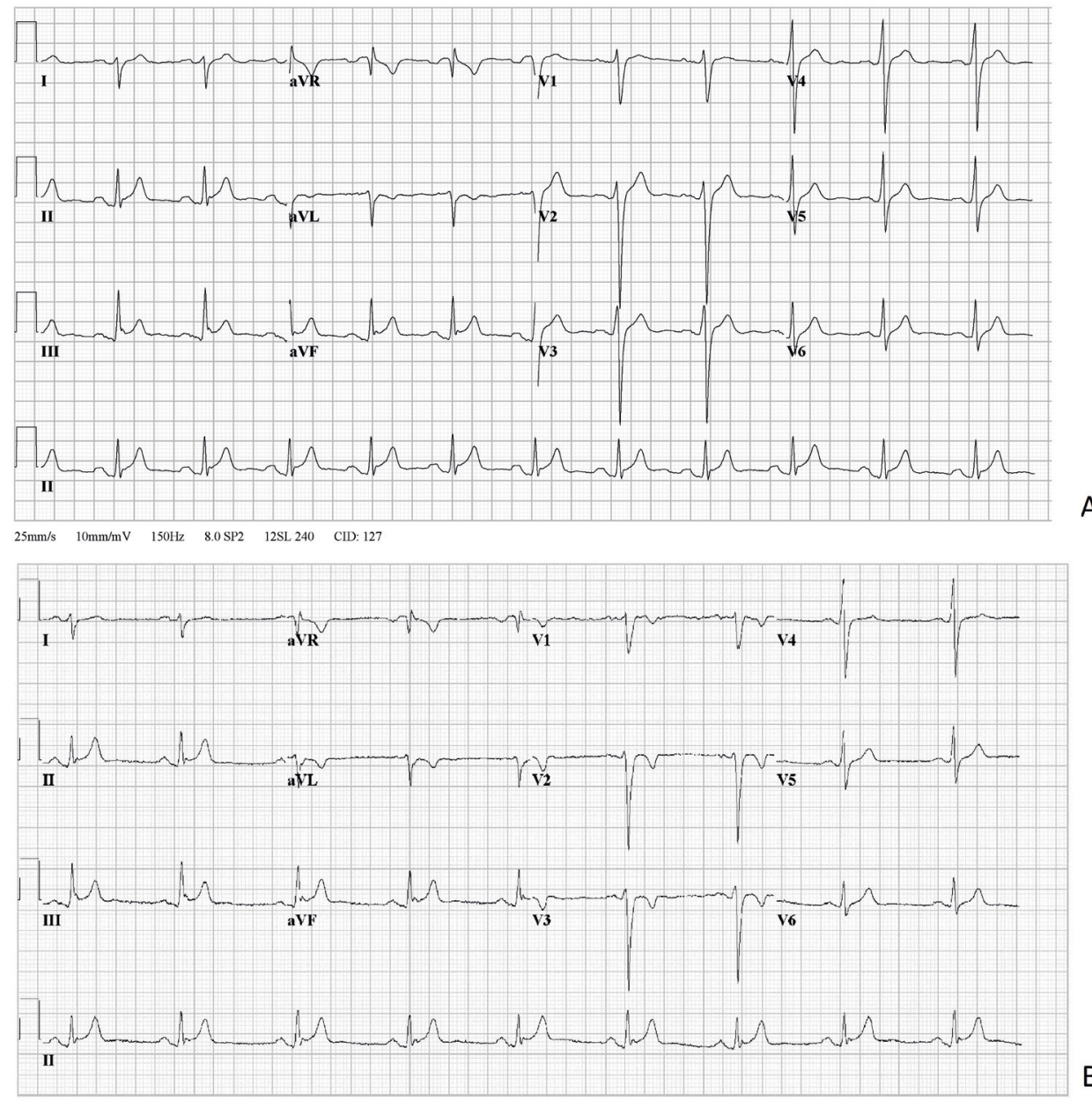

Fig. 1 12-lead electrocardiogram while off anti-arrhythmic drugs, showing normal sinus rhythm, QRS right axis deviation, QRS width $140 \mathrm{~ms}$ and prolonged terminal activation duration in V2 (70ms). Clear J-point and ST elevation in II, III, and aVF.

During twenty-five years of follow-up, he remained free of ventricular tachyarrhythmias. Ten years after the initial event negative $T$ waves were recorded in V1-V3 (Fig. 2). On follow-up echocardiogram, there were no longer signs of tricuspid valve prolapse, but careful re-evaluation of the imaging showed subtricuspid dyskinesia. Sixteen years after the event 
right ventricular dilatation was found (echocardiogram 2008: dilated right ventricle (RV), regional hypokinesia of the right ventricular outflow tract (RVOT) and anterior right ventricle, parasternal long axis view RVOT: $32 \mathrm{~mm}$, parasternal short axis view RVOT: 35mm, Fig. 3). Consecutive targeted molecular genetic testing revealed no pathogenic mutations in desmosomal genes, but the sodium channel gene SCN5A revealed a p. Leu729del mutation. Familial co-segregation supported pathogenicity of this mutation ${ }^{14}$.

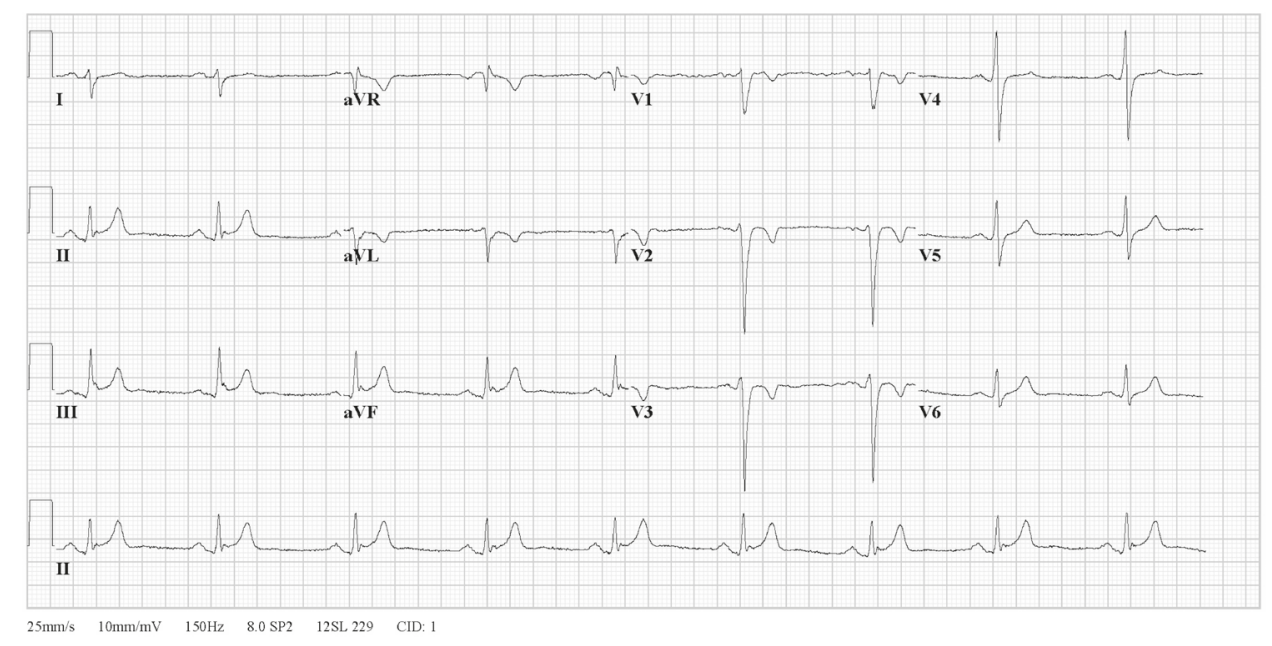

Fig. 2 12-lead electrocardiogram while off anti-arrhythmic drugs after 10 years of follow-up, showing negative $T$ waves in V1-V3. Terminal activation delay and J-point elevation are still present. 


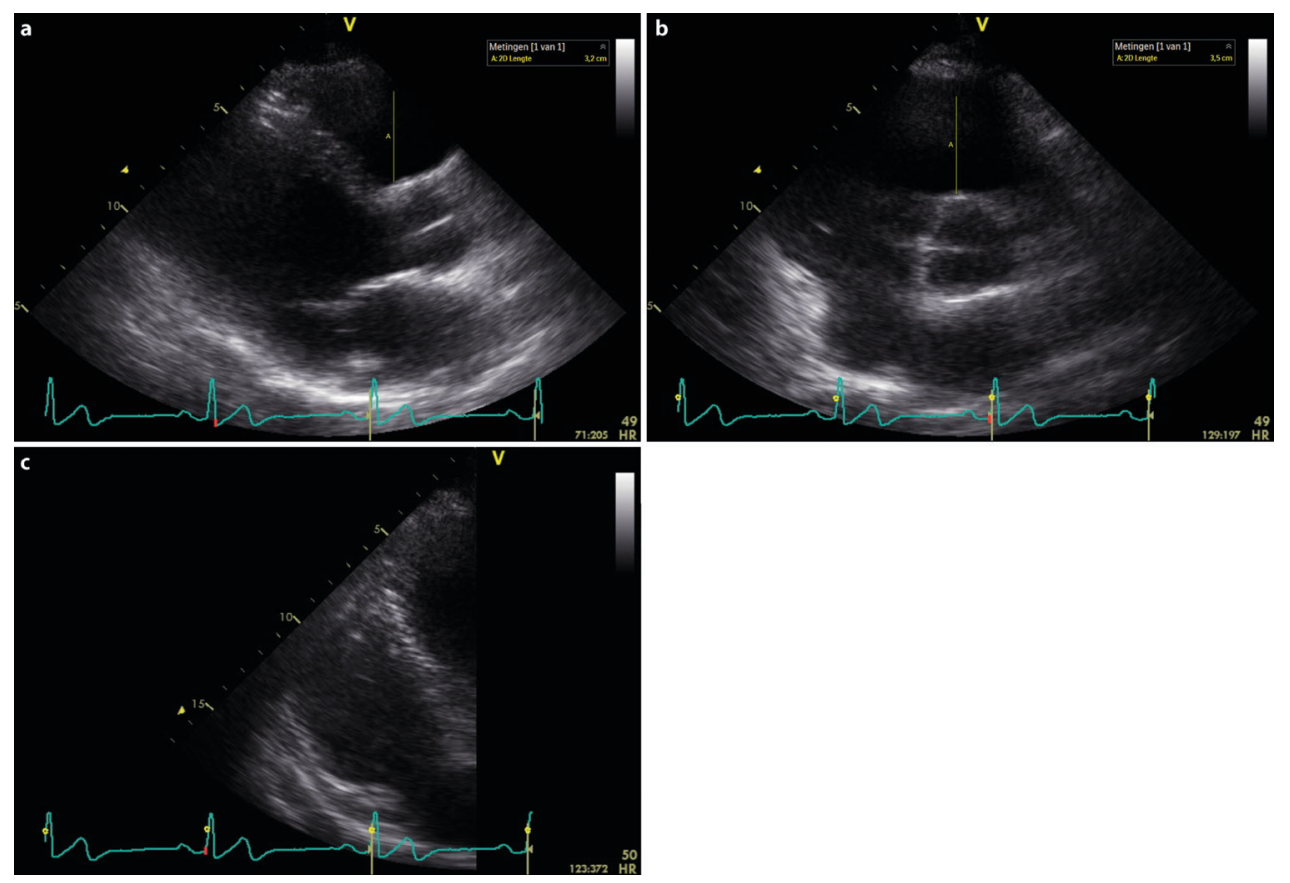

Fig. 3 Echocardiogram in 2008 showing dilatation of the right ventricle and right ventricular outflow tract. a PLAX RVOT, b PSAX RVOT, c AP4CHfocused RV. PLAX parasternal long axis view, RVOT right ventricular outflow tract, PSAX parasternal short axis view, $A P 4 C H$ apical 4 chamber view, $R V$ right ventricle

After re-evaluation of the diagnostic workup he was diagnosed with arrhythmogenic cardiomyopathy according to the 2010 International Task Force Criteria ${ }^{15}$ : T-wave inversion in right precordial leads (1 major), ventricular tachycardia with left bundle branch block configuration and superior axis (1 major), structural abnormalities on echocardiogram (1 major) and prolonged terminal activation duration (1 minor). As this case shows, the diagnosis of underlying disease in idiopathic ventricular fibrillation is a dynamic process in which the initial evaluation has not led to a satisfactory result and over time underlying disease can become manifest. During long-term follow-up, conventional diagnostic tests can detect progression of underlying disease, new diagnostic tests 
can aid in detecting underlying disease and increased understanding of the disease can lead to diagnosis and additional management options.

\section{FUTURE PERSPECTIVES IN IDIOPATHIC VENTRICULAR FIBRILLATION RESEARCH}

\section{The role of a national/international database}

The current population with idiopathic ventricular fibrillation is a rare, heterogeneous group, where diagnostic certainty depends on the extent of workup to exclude other underlying causes. The prevalence of idiopathic ventricular fibrillation in the Netherlands is unknown, but it is estimated at 1,000 patients nationwide. At this moment, little is known about the diagnostic certainty and extent of diagnostic workup of patients with idiopathic ventricular fibrillation in the Netherlands. A comprehensive registry of patients with idiopathic ventricular fibrillation with national and international coverage should be established to study the prevalence and characteristics of these patients. The database should include information on performed diagnostic tests to assess the reliability of the diagnosis and clues for underlying disease.

Through advances in the understanding of primary electrical syndromes and the discovery of novel founder mutations in idiopathic ventricular fibrillation, subgroups with a specific underlying disease can be identified and diagnosed and managed appropriately, improving prognosis and preventing recurrent events.

\section{Design of the national consortium study VIGILANCE under the Netherlands Cardiovascular Research Initiative}

The VIGILANCE (non-invasive electrocardiographic imaging for individuals at risk for apparently idiopathic ventricular fibrillation) project focuses on establishing a Dutch national registry of patients with idiopathic ventricular fibrillation. The registry's foundation has been laid and already includes 8 university medical centres and tertiary centres. Our aim is to include all Heart Centres of the University Medical Centres and all ICD implant centres in the Netherlands. We will visit these institutes and collect 


\section{6 | CHAPTER 2}

patients from existing electronic patient records. In the beginning of the inclusion process, survivors of cardiac arrest due to ventricular fibrillation in whom at least $50 \%$ of routine diagnostic tests have been performed and no underlying disease has been found will be included in the registry. These survivors will be screened using the diagnostic flowchart published by our group (Fig. 4) and comprehensive data review will be performed to assess the completeness of diagnostic testing. Next, we will discuss the missing diagnostics with the treating physician and finalise the diagnostic process, excluding patients with alternative diagnoses. From this cohort of patients with clear idiopathic ventricular fibrillation with complete diagnostic workup, we will collect data on long-term follow-up of patients with idiopathic ventricular fibrillation, including ICD therapy and clinical outcome. Furthermore, this registry will be a starting point for future research on the arrhythmogenesis of idiopathic ventricular fibrillation.

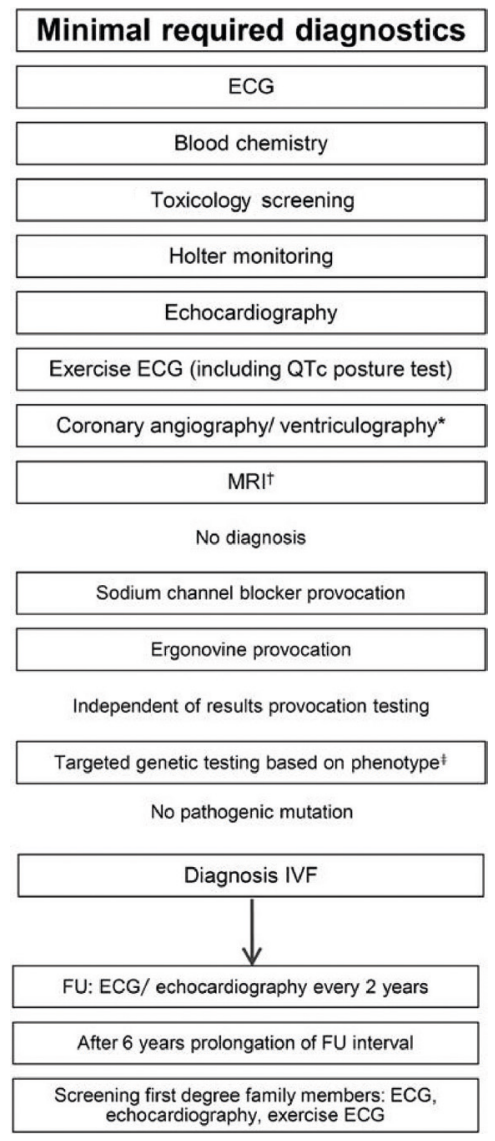


Fig. 4 Flowchart for the diagnosis and follow-up of patients with idiopathic ventricular fibrillation. ${ }^{*}$ In young patients ( $<45$ years) without risk factors for coronary artery disease, coronary computed tomography (CT) angiography is an alternative diagnostic tool to exclude coronary artery disease. ECG electrocardiogram, IVF idiopathic ventricular fibrillation, MRI magnetic resonance imaging, $F U$ follow-up (from Visser et al. ${ }^{2}$ )

\section{Follow-up of patients with idiopathic ventricular fibrillation}

During follow-up, patients will be monitored continuously and undergo diagnostic tests at regular intervals, which will provide valuable insight into natural disease course, specific subgroup identification and disease severity. Data on the appropriate burden of ICD shock and ICD-related complications can be used to assess the need and benefit of ICD implantation.

\section{Genetics and family screening}

Arrhythmic risk assessment in asymptomatic patients is difficult, especially with unknown underlying disease. However, many patients with idiopathic ventricular fibrillation show a familial risk for sudden cardiac death and there are several well-characterised populations (e.g. DPP6, SCN5A) in the Netherlands ${ }^{6,16}$. Data from genetic testing in patients with idiopathic ventricular fibrillation and their family members will be used to assess the yield of genetic testing, the detection of variants of uncertain clinical significance and their contribution to arrhythmic risk in this population.

\section{Body surface mapping}

Absence of known arrhythmic substrates in patients with idiopathic ventricular fibrillation provides an opportunity for novel, preferably noninvasive, techniques. Electrocardiographic imaging (ECGI) is an emerging high-resolution non-invasive imaging modality for mapping the electrical activity in the heart ${ }^{17,18}$. ECGI employs mathematical formulations to reconstruct the electrical activity at the level of the heart muscle, from extensive body-surface electrocardiograms and a digital, patient-specific 


\section{8 | CHAPTER 2}

body and heart geometry. It uses a model of the propagation of the electromagnetic field (from the heart to the body surface) to reconstruct the electrical source of the recorded body-surface ECGs ${ }^{19}$. In healthy individuals, ECGI demonstrated that dispersion is small, for example for repolarisation in humans ${ }^{20,21}$. However, ECGI did show increased dispersion in several arrhythmogenic diseases. In long QT syndrome ${ }^{20}$ and Brugada syndrome ${ }^{21}$, patients had abnormally steep repolarisation gradients. Repolarisation gradients were steeper in symptomatic patients, suggesting increased dispersion is predictive for arrhythmia risk ${ }^{20}$. We have validated ECGI's ability to assess depolarisation and repolarisation accurately. These results suggest ECGI can play a pivotal role in characterising arrhythmia mechanisms in patients with idiopathic ventricular fibrillation, leading to diagnosis and improved treatment. Moreover, it seems to have the potential to detect arrhythmogenic substrate in individuals before their first event, offering the possibility to diagnose and treat patients before sudden cardiac arrest occurs. This is especially relevant in family members of victims of sudden cardiac death who might have a predisposition for idiopathic ventricular fibrillation. Within the VIGILANCE project, we will screen patients with idiopathic ventricular fibrillation for arrhythmogenic substrate using ECGI and help identify family members at risk for arrhythmic events. 


\section{References}

1. Priori SG, Wilde AA, Horie M, et al. HRS/EHRA/APHRS Expert consensus statement on the diagnosis and management of patients with inherited primary arrhythmia syndromes. Heart Rhythm. 2013;10:1932-63.

2. Visser M, van der Heijden JF, Doevendans PA, et al. Idiopathic ventricular fibrillation: The struggle for definition, diagnosis and followup. Circ. Arrhythmia Electrophysiol. 2016;9:e3817.

3. Krahn AD, Healey JS, Chauhan V, et al. Systematic assessment of patients with unexplained cardiac arrest: Cardiac Arrest Survivors With Preserved Ejection Fraction Registry (CASPER). Circulation. 2009;120:278-85.

4. Honarbakhsh S, Srinivasan N, Kirkby C, et al. Medium-term outcomes of idiopathic ventricular fibrillation survivors and family screening: a multicentre experience. Europace. 2016;19:1874-80.

5. Visser M, Dooijes D, van der Smagt J], et al. Next-generation sequencing of a large gene panel in patients initially diagnosed with idiopathic ventricular fibrillation. Heart Rhythm. 2017;14:1035-40.

6. ten Sande JN, Postema PG, Boekholdt SM, et al. Detailed characterization of familial idiopathic ventricular fibrillation linked to the DPP6 locus. Heart Rhythm. 2016; 13:905-12.

7. Champagne J, Geelen P, Philippon F, Brugada P. Recurrent cardiac events in patients with idiopathic ventricular fibrillation, excluding patients with the Brugada syndrome. BMC Med. 2005;3:1.

8. Remme CA, Wever EFD, Wilde AAM, et al. Diagnosis and longterm follow-up of the Brugada syndrome in patients with idiopathic ventricular fibrillation. Eur Heart J. 2001;22:400-9.

9. Herman ARM, Cheung C, Gerull B, et al. Outcome of apparently unexplained cardiac arrest: Results from investigation and follow-up of the prospective Cardiac Arrest Survivors With Preserved Ejection Fraction Registry. Circ Arrhythm Electrophysiol. 2016;9:e3619.

10. Matassini VM, Krahn AD, Gardner M, et al. Evolution of clinical diagnosis in patients presenting with unexplained cardiac arrest or 
syncope due to polymorphic ventricular tachycardia. Heart Rhythm. 2014;11:274-81.

11. Conte G, Caputo ML, Regoli F, et al. True idiopathic ventricular fibrillation in out-of-hospital cardiac arrest survivors in the Swiss Canton Ticino: prevalence, clinical features, and long-term followup. Europace. 2017;19:259-66.

12. Visser M, van der Heijden JF, van der Smagt JJ, et al. Long-term outcome of patients initially diagnosed with idiopathic ventricular fibrillation. Circ. Arrhythmia Electrophysiol. 2016;9:e4258.

13. Mewis C, Kuhlkamp V, Spyridopoulos I, et al. Late outcome of survivors of idiopathic ventricular fibrillation. Am J Cardiol. 1998;81:999-1003.

14. te Riele ASJM, Agullo-Pascual E, James CA, et al.Multilevel analyses of SCN5A mutations in arrhythmogenic right ventricular dysplasia/ cardiomyopathy suggest non-canonical mechanisms for disease pathogenesis. Cardiovasc Res. 2017;113:102-11.

15. Marcus FI, McKenna WJ, Sherrill D, et al. Diagnosis of arrhythmogenic right ventricular cardiomyopathy/dysplasia: Proposed modification of the task force criteria. Circulation. 2010;121:1533-41.

16. Ter Bekke RMA, Isaacs $A$, Barysenka $A$, et al. Heritability in a SCN5Amutation founder population with increased female susceptibility to non-nocturnal ventricular tachyarrhythmia and sudden cardiac death. Heart Rhythm. 2017;14:1873-81.

17. Ramanathan C, Ghanem RN, Jia P, et al. Noninvasive electrocardiographic imaging for cardiac electrophysiology and arrhythmia. Nat Med. 2004;10:422-8.

18. Cluitmans MJM, Peeters RLM, Westra RL, Volders PGA. Noninvasive reconstruction of cardiac electrical activity: Update on current methods, applications and challenges. Neth Heart J. 2015;23:301-11.

19. Cluitmans MJM, Clerx M, Vandersickel N, et al. Physiology-based regularization of the electrocardiographic inverse problem. Med Biol Eng Comput. 2017;55:1353-65.

20. Vijayakumar R, Silva JNA, Desouza KA, et al. Electrophysiologic Substrate in Congenital Long QT Syndrome: Noninvasive Mapping With 
Electrocardiographic Imaging (ECGI). Circulation. 2014;130:1936-43.

21. Zhang J, Sacher F, Hoffmayer K, et al. Cardiac Electrophysiological Substrate Underlying the ECG Phenotype and Electrogram Abnormalities in Brugada Syndrome Patients. Circulation. 2015;131:1950-9. 


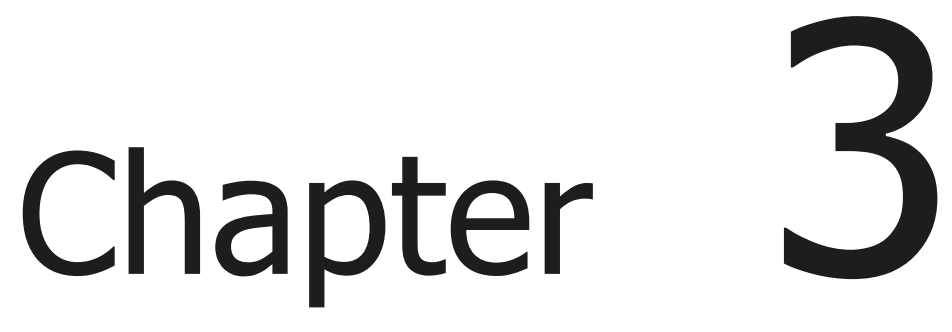

\title{
Sudden Cardiac Death in the Young: Post-mortem Investigation and Cardiogenetic Evaluation of Victims and Their Relatives.
}

\author{
L.J. Blom¹, A.F. Baas², A. Vink³, R.J. Hassink ${ }^{1}$
}

1 Department of Cardiology, University Medical Center, Utrecht, The Netherlands

2 Department of Clinical Genetics, University Medical Center, Utrecht, The Netherlands

3 Department of Pathology, University Medical Center, Utrecht, The Netherlands

Clinical Cardiogenetics - in press 


\section{4 | CHAPTER 3}

\section{Abstract}

Sudden cardiac death in the young is a rare and devastating event. A dedicated and focused post-mortem investigation is essential in detecting potential inherited cardiac diseases in sudden death victims, which facilitates appropriate management of the victim's family members. Many specialized clinical disciplines are involved and a multidisciplinairy approach is required to provide the best outcome in these cases. 


\section{INTRODUCTION}

Sudden cardiac death (SCD) was recently defined as "Sudden and unexpected death occurring within an hour of the onset of symptoms, or occurring in patients found dead within $24 \mathrm{~h}$ of being asymptomatic and presumably due to a cardiac arrhythmia or hemodynamic catastrophe". ${ }^{1}$ Population studies show that over two-third of sudden death cases are of suspected cardiac origin and classified as SCD. ${ }^{2}$

The incidence of SCD in persons aged $1-40$ years varies depending on the population studied and methodology used. The incidence for children is 1.3 per 100.000 person-years and increases to 8.5 per 100.000 in adults up to 40 years of age. ${ }^{3}$

The underlying causes identified in autopsy series suggest that in people under 35 years of age, $24-31 \%$ of deaths are due to coronary artery disease, $17-37 \%$ are associated with cardiomyopathy, predominantly hypertrophic cardiomyopathy, idiopathic left ventricular hypertrophy and arrhythmogenic cardiomyopathy (depending on the investigated region ${ }^{4}$ ) and in $31-35 \%$ no cause is found by gross and histological examination and the death is presumed to be arrhythmic. ${ }^{5,6}$ Due to the probability of inherited disease in these cases, up to $50 \%$ in SCD victims without structural heart disease, ${ }^{7}$ post-mortem diagnosis in the victim is crucial for appropriate management of the victim's family members.

In practice, not all causes of SCD in a young victim are established. Usually, the victim was asymptomatic and the event is the first presentation of the disease or the victim suffered from non-specific symptoms, which often are not formally evaluated. The deaths are frequently unwitnessed and circumstances at the time of death have to be retrieved from ambulance and police reports. ${ }^{3}$

When autopsy cannot identify a cause, which happens in up to $30 \%,{ }^{6}$ these cases are referred to as Sudden arrhythmic death syndrome (SADS). Despite the absence of an apparent underlying cause, the possibility of an underlying inherited cardiac disease remains and family members should be considered for cardiogenetic consultation and cardiac evaluation. 


\section{AFTER THE EVENT}

A dedicated and focused post-mortem investigation is essential in detecting potential inherited cardiac diseases in sudden death victims. This involves gathering as much information about the deceased as possible. The investigation should include obtaining a premorbid medical history, occurrence of syncope, exertional symptoms, prior illness, recently prescribed medication and any previous ECGs or other diagnostic tests performed. Circumstances of SCD often rely on obtaining information from available ambulance and police reports. When no further investigations are performed, these cases are referred to as Sudden unexplained death (SUD).

Family history is another important part of the post-mortem investigation and should contain a comprehensive three-generation family pedigree focused on identifying a family history of cardiac disease and premature sudden unexplained death. Examples of suspicious symptoms or deaths in family members are sudden infant death syndrome (SIDS), epilepsy, recurrent fainting, drowning of experienced swimmers in (shallow) waters or one-sided vehicle accidents. ${ }^{3,8}$

The recommended steps to be taken after the event are presented in figure 1 .

\section{AUTOPSY AND TOXICOLOGICAL SCREENING}

A full post-mortem examination is strongly recommended in all cases of sudden death in the young (between 1 - 40 years old), as this represents the first, and only, opportunity to establish and register an accurate cause of death. The international guideline for post-mortem investigation and autopsy in SCD/SUD provides a minimum standard that is required in the routine autopsy practice for the adequate investigation of SCD. ${ }^{9}$ If little experience with cardiac examination is available referral of the heart(tissue) to a cardiac expert pathologist is recommended. In the report of the autopsy, the pathologist should state the certainty to which 
the findings of the autopsy could have led to the sudden death, since findings of uncertain significance might lead to erroneous interpretation and e.g. may mask underlying disease. ${ }^{10}$ During the autopsy appropriate material for toxicology, microbiology, biochemistry, and molecular investigation should be obtained. A toxicological examination for drugs (e.g., opiates, amphetamine), alcohol, and medication may be considered necessary in cases with no structural abnormalities at autopsy, although the presence of drugs does not rule out an underlying genetic disease..$^{11,12}$ Over $70 \%$ of SCD autopsies yield an underlying diagnosis and are thereby the most effective post-mortem investigation in young SCD victims. ${ }^{2,5,6}$ Post-mortem diagnosis may give psychological closure for the family. ${ }^{13}$ In case a potential inherited disorder was found, this may give rise to cascade screening of family members and preventive management. Autopsy findings that indicate involvement of other inherited disease, such as premature coronary artery disease in familial hypercholesterolemia or genetically vulnerable myocardium which may predispose for myocarditis should also be considered. When no diagnosis could be made, autopsy findings can be used to guide screening and management of family members. ${ }^{10}$ 


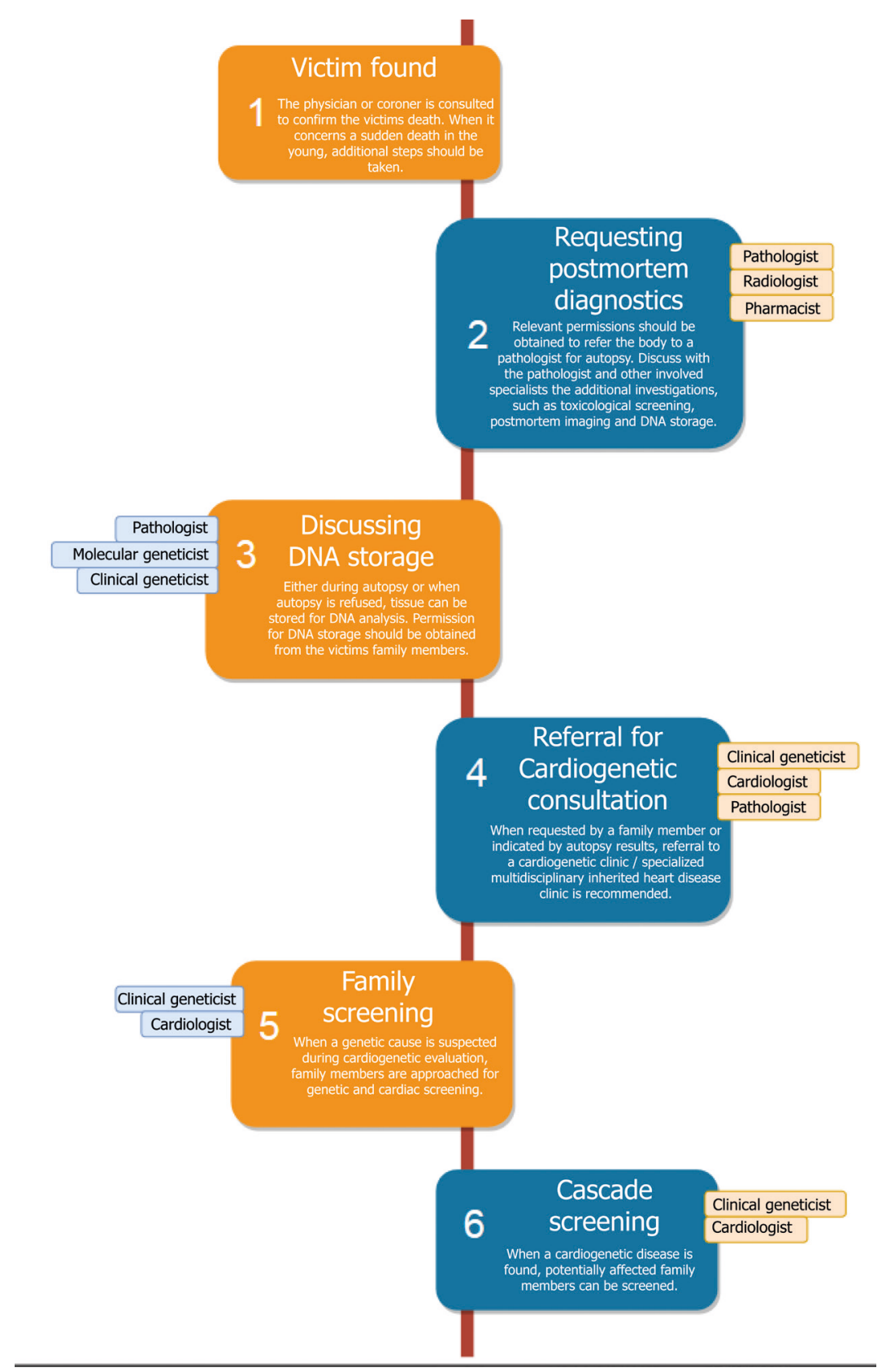

Figure 1. Flowchart of recommended actions and investigations after a sudden death in the young suspected of inherited heart disease, including the multidisciplinary specialists involved. SCD Sudden cardiac death. 


\section{POST-MORTEM IMAGING}

Non-invasive imaging modalities may aid in improving diagnostic accuracy when autopsy is not performed. Post-mortem imaging alone, also known as 'virtual' or 'minimally invasive' autopsy, is as of now insufficient as a substitute for conventional autopsy, but can be an alternative when conventional autopsy is e.g. refused by the victim's relatives. ${ }^{14}$ Both postmortem computer tomography (CT) and cardiac magnetic resonance imaging (CMR) can be performed within a few hours after death. Postmortem CT usually consists of a scan of head and neck, thorax and abdomen and should be interpreted by a radiologist with experience regarding post-mortem imaging techniques. Post-mortem MR imaging is generally dedicated to the heart and brain to detect more subtle abnormalities that could have been the cause of death.

The utility and yield of post-mortem imaging in SCD is still unclear. An Australian investigation compared the outcome of regular post-mortem investigation with post-mortem MRI and CT in 17 SCD victims and showed that MRI had a high sensitivity and positive predictive value compared to traditional autopsy for arrhythmogenic cardiomyopathy, ischemic heart disease, pulmonary embolism or aortic dissection and could be used as a rule-out when autopsy is not possible. ${ }^{15}$ Another issue is cost assessment, which is complicated as mortuary service and post-mortem imaging costs vary considerably between and within countries. A recent report estimated post-mortem imaging could increased costs for post-mortem investigation by $>30 \%$ and propose a national service for cost-effective implementation. ${ }^{16}$ In general, costs of post-mortem investigation are paid by the initiator. When post-mortem investigation is required by law, for example in unnatural death, the state will refund the costs. When the death occurs in hospital, the post-mortem investigations may be financed by the hospital. Post-mortem investigation of out-of-hospital deaths is usually funded by the family of the deceased. Sudden death of a young individual is a reason for further investigation, but in many countries is still funded by the family when the death occurs out-of-hospital. In the UK it is legally required to perform post-mortem investigations in these cases, 
and therefore the costs are paid by the state. To improve the rate of postmortem investigation of young SCD victims in other countries, funding by state or health insurance companies should be considered.

\section{MOLECULAR AUTOPSY}

In SCD victims, post-mortem investigation allows tissue to be collected and stored in the way it is recommended by pathology guidelines. When a hereditary cause of death is suspected, the pathologist can recommend referral of first-degree family members to a cardiogenetic clinic and store DNA of the deceased for potential genetic testing. ${ }^{9}$ Even when autopsy is refused, a minimal amount of tissue can be collected from the victim for storage. This tissue can be used for genetic testing. The types of tissue that are usually collected during autopsy are EDTA-blood and/or frozen (cardiac) muscle, liver, or spleen. ${ }^{17,18}$ A skin biopsy can be taken when no autopsy is performed (with permission from the victim's relatives). Prior to the biopsy, the skin needs to be disinfected with alcohol. The obtained tissue can be temporarily stored in a sterile vial with physiological isotonic saline before sending it to a DNA laboratory. ${ }^{19}$

Storage of the victim's DNA enables genetic testing when relatives consult a cardiologist or geneticist for cardiogenetic evaluation. The yield of post-mortem genetic testing in SCD victims is modest $13 \%$ in Lahrouchi et $\mathrm{al}^{20} 27 \%$ in Tan et $\mathrm{al}^{21}$ and $32 \%$ in Kumar et al ${ }^{22}$, however when combined with clinical evaluation of relatives the diagnostic yield increases to approximately $40 \% .^{21,23}$ The recommended number of genes in molecular autopsy panels is under debate, as the ratio of rare variants of uncertain significance (VUS) to pathogenic or likely pathogenic variants is unfavourable. Therefore, pretest counselling of the relatives is essential. The HRS/EHRA consensus states that comprehensive or targeted (KCNQ1, $\mathrm{KCNH} 2$, SCN5A, and RyR2) gene testing may be considered in unexplained sudden death cases, where they account for $35 \%$ of pathologic mutations found. ${ }^{8,19}$ Further genetic testing should be guided, but not limited by phenotypic findings in the deceased, as even in autopsy negative SADS patients, pathogenic variants are found in cardiomyopathy associated 
genes. ${ }^{10}$

Together with the clinical evaluation of the family, post-mortem genetic testing (i.e. a molecular autopsy) could uncover the cause of death in the deceased. When a genetic cause is found, cascade screening of at-risk family members can be initiated.

\section{CARDIOGENETIC EVALUATION OF FIRST-DEGREE RELATIVES OF YOUNG SCD VICTIMS}

\section{Phenotypic screening}

In most cases of SCD phenotypic screening of relatives is indicated. The extent of this screening is dependent on the findings in the deceased. These findings can then be used for targeted genetic testing in the victim (if the victim's DNA is available) or in the clinically affected relative (with cardiac abnormalities).

When there are no clues available for a specific diagnosis, in SADS cases for example, phenotypic screening is important to reveal inherited disease in the family. First-degree relatives, obligate carriers, and symptomatic relatives should be evaluated.

A cardiac examination of the relatives should follow a standard approach and is based largely on the HRS/EHRA consensus document for inherited heart diseases. ${ }^{8}$ Examination should include the following aspects; (1) medical and family history, (2) physical examination, (3) standard resting 12 lead electrocardiogram, 12 lead electrocardiogram after brisk standing ${ }^{24}$ and 12 lead electrocardiogram with specific right precordial positioning of the leads (leads - V1, V2, 1V1, and $1 \mathrm{~V} 2$ ), ${ }^{25}$ (4) exercise testing, and (5) echocardiography. If the initial examination raises the possibility of a specific genetic disorder, further investigations may be indicated, which may include provocation testing (e.g., ajmaline challenging in suspected Brugada Syndrome patients), cardiac MRI, Holter recording, or signalaveraged ECG.3,8

\section{Mutation-positive SCD victim}

When a genetic mutation is found in the stored DNA of the SCD victim, 
as assessed after a first-degree relative has requested cardiogenetic screening, the pathogenicity of the particular mutation must be assessed. In case of a pathogenic (disease-causing) mutation, the presence of the mutation is enough to diagnose inherited disease. Confirmation or exclusion of the presence of a disease-causing mutation in presymptomatic family members of the sudden death victim by cascade screening will guide risk stratification and management. ${ }^{8,13}$ Because most inherited heart diseases show an autosomal dominant pattern of inheritance, first-degree relatives (parents, siblings and children) of SCD victims with genetic disease have a $50 \%$ risk of being a carrier of the same disease. ${ }^{26}$ This is not the case if a mutation occurred de novo in the victim (is absent in the parents). However, due to the low probability of a germline mosaicism, siblings are still eligible for genetic screening in case of a de novo mutation.

Cascade screening starts with genetic testing of the (genetically) firstdegree relatives of an affected individual or second-degree relatives when a first-degree relative has deceased. Subsequently, the screening can be extended to the connecting branch of the pedigree. ${ }^{27}$ The absence of the mutation rules out the presence of the disease, and no further testing of the connecting pedigree is needed. In case a causative mutation is present in a relative, cardiologic evaluation and/or diagnostic follow-up is usually indicated.

In case there is uncertainty about the significance of the discovered mutation (class 2-4), the results should be interpreted together with the outcome of phenotypic screening of relatives to determine the probability of inherited disease in the family members.

\section{Mutation-negative SCD victim}

Evaluation of family members of SCD victims in whom no genetic diagnosis is established is complex and should preferably involve a multidisciplinary team of specialists. Therefore, cardiogenetic evaluation is best performed in the context of referral of family members to a cardiogenetic clinic. ${ }^{3,8}$ Cascade screening should only be performed together with clinical evaluation of the family and preferably guided by 
post-mortem findings in the deceased.

The yield of genetic screening in family members varies depending on the phenotypic screening performed and the results of post-mortem investigation in the SCD victim. If these provide clues for cardiac disease, in more than $5 \%$ of first-degree relatives a disease-causing mutation is found. Because of incomplete penetrance and variable expression, the subsequent management and follow-up of relatives differs between diagnoses. The genetic test results must be interpreted cautiously and incorporated in results of clinical evaluation. ${ }^{8}$ If no diagnosis is made after comprehensive evaluation, then asymptomatic relatives are generally followed up till age 40 years. ${ }^{3}$

\section{Yield of cardiogenetic evaluation}

With thorough clinical assessment of first-degree relatives of SUD victims, a cause of death can be established in up to $50 \%$ of selected and comprehensively evaluated families. ${ }^{21,23,28}$ A Dutch investigation of 43 families of SUD victims, of whom 22 were autopsied, found an inherited cardiac disease in 17 of the 43 families that explained the sudden death of the victims. ${ }^{21}$ Furthermore, a study executed in the United Kingdom revealed an inherited disease in $53 \%$ of the 57 families of SADS victims aged 4-64 years. ${ }^{23}$

Discovery of inherited disease in an (asymptomatic) relative of a SCD victim does not necessarily have prognostic consequences. Nor does the absence of abnormalities on cardiac evaluation automatically rule out the presence of an inherited disease.

This complicates the management of relatives and requires a disease specific approach. In some diseases, symptoms develop only at older age (e.g., HCM, DCM, and ARVD/C), which may mandate follow-up in these individuals. In others, an extensive cardiogenetic evaluation is sufficient for diagnosis or exclusion (e.g., LQTS, BrS and CPVT). However, it should be realized that a relative carrying the mutation of an inherited cardiac disease will not necessarily develop signs or symptoms of the clinical syndrome that is associated with the mutation. 


\section{4 | CHAPTER 3}

\section{CONCLUSION}

Sudden cardiac death in the young is a devastating event that deserves thorough investigation and care for the benefit of the victims family members. Many specialized clinical disciplines need to collaborate to perform these investigations and outcome is dependent on the coordinated efforts of all those involved. A comprehensive overview of the different clinical scenarios and recommended management options is presented in the flowchart in figure 2. 

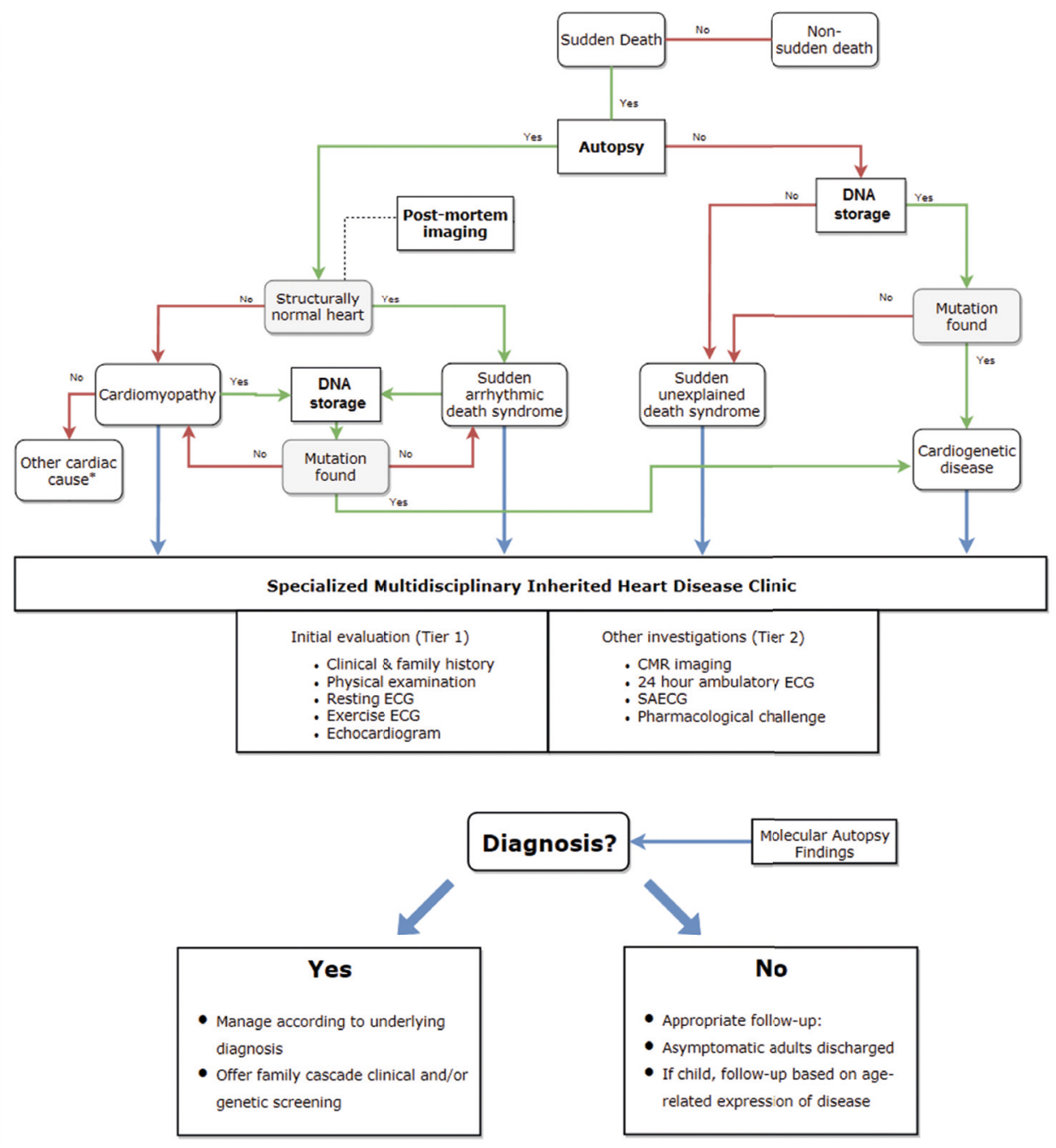

Figure 2. Flowchart of diagnosis and management of inherited heart disease in families of a young sudden death victim. Note: DNA testing of stored DNA sample is initiated only after the relatives visit the outpatient clinic and request on DNA testing. *Inherited disease may be suspected, see paragraph 'autopsy and toxicological screening'. ECG Electrocardiogram; CMR cardiac magnetic resonance imaging; SAECG Signal-averaged electrocardiogram. Adapted from Semsarian et $a^{3}$. 


\section{References}

1. Al-Khatib SM, Stevenson WG, Ackerman MJ, et al (2017) 2017 AHA/ ACC/HRS Guideline for Management of Patients With Ventricular Arrhythmias and the Prevention of Sudden Cardiac Death: Executive Summary. Hear Rhythm. doi: 10.1016/j.hrthm.2017.10.035

2. Winkel BG, Holst AG, Theilade J, Kristensen IB, Thomsen JL, Ottesen GL, Bundgaard H, Svendsen JH, Haunsø S, Tfelt-Hansen J (2011) Nationwide study of sudden cardiac death in persons aged 1-35 years. Eur Heart J 32:983-990

3. Semsarian C, Ingles J, Wilde AAM (2015) Sudden cardiac death in the young: The molecular autopsy and a practical approach to surviving relatives. Eur Heart J 36:1290-1296

4. Sheppard MN (2012) Aetiology of sudden cardiac death in sport: A histopathologist's perspective. Br J Sports Med 46:15-21

5. Eckart RE, Shry EA, Burke AP, et al (2011) Sudden death in young adults: An autopsy-based series of a population undergoing active surveillance. J Am Coll Cardiol 58:1254-1261

6. Doolan A, Langlois N, Semsarian C (2004) Causes of sudden cardiac death in young Australians. Med J Aust 180:110-2

7. Ranthe MF, Winkel BG, Andersen EW, Risgaard B, Wohlfahrt J, Bundgaard H, Haunsø S, Melbye M, Tfelt-Hansen J, Boyd HA (2013) Risk of cardiovascular disease in family members of young sudden cardiac death victims. Eur Heart ] 34:503-511

8. Ackerman MJ, Priori SG, Willems S, et al (2011) HRS/EHRA Expert Consensus Statement on the State of Genetic Testing for the Channelopathies and Cardiomyopathies. Hear Rhythm 8:1308-1339

9. Basso C, Aguilera B, Banner J, et al (2017) Guidelines for autopsy investigation of sudden cardiac death: 2017 update from the Association for European Cardiovascular Pathology. Virchows Arch 471:691-705

10. Papadakis M, Raju H, Behr ER, De Noronha S V., Spath N, Kouloubinis A, Sheppard MN, Sharma S (2013) Sudden Cardiac Death With Autopsy Findings of Uncertain Significance: Potential for Erroneous 
Interpretation. Circ Arrhythmia Electrophysiol 6:588-596

11. Tominaga $M$, Michiue $T$, Inamori-Kawamoto $O$, Hishmat AM, Oritani S, Takama M, Ishikawa T, Maeda H (2015) Efficacy of drug screening in forensic autopsy: Retrospective investigation of routine toxicological findings. Leg Med 17:172-176

12. Bjune $T$, Risgaard $B$, Kruckow $L$, Glinge $C$, Ingemann-Hansen $O$, Leth PM, Linnet K, Banner J, Winkel BG, Tfelt-Hansen J (2018) Post-mortem toxicology in young sudden cardiac death victims: a nationwide cohort study. EP Eur 20:614-621

13. Tester DJ, Ackerman MJ (2017) Evaluating the survivor or the relatives of those who do not survive: The role of genetic testing. Cardiol Young 27:S19-S24

14. Sonnemans LJP, Kubat B, Prokop M, Klein WM (2018) Can virtual autopsy with postmortem CT improve clinical diagnosis of cause of death? A retrospective observational cohort study in a Dutch tertiary referral centre. BMJ Open 8:e018834

15. Puranik R, Gray B, Lackey H, Yeates L, Parker G, Duflou J, Semsarian C (2014) Comparison of conventional autopsy and magnetic resonance imaging in determining the cause of sudden death in the young. J Cardiovasc Magn Reson 16:1-11

16. Karalis J, Denton E (2016) Forensic and post-mortem imaging in England: A national perspective. J Forensic Radiol Imaging 4:17-19

17. Priori SG, Blomström-Lundqvist C, Mazzanti A, et al (2015) 2015 ESC Guidelines for the management of patients with ventricular arrhythmias and the prevention of sudden cardiac death. Eur Heart J 36:2793-2867

18. Carturan E, Tester DJ, Brost BC, Basso C, Thiene G, Ackerman MJ (2008) Postmortem Genetic Testing for Conventional Autopsy-Negative Sudden Unexplained Death. Am J Clin Pathol 129:391-397

19. Semsarian C, Hamilton RM (2012) Key role of the molecular autopsy in sudden unexpected death. Hear Rhythm 9:145-150

20. Lahrouchi N, Raju H, Lodder EM, et al (2017) Utility of Post-Mortem Genetic Testing in Cases of Sudden Arrhythmic Death Syndrome. J Am Coll Cardiol 69:2134-2145 
21. Tan HL, Hofman N, Van Langen IM, Van Der Wal AC, Wilde AAM (2005) Sudden unexplained death: Heritability and diagnostic yield of cardiological and genetic examination in surviving relatives. Circulation 112:207-213

22. Kumar S, Peters S, Thompson T, Morgan N, Maccicoca I, Trainer A, Zentner D, Kalman JM, Winship I, Vohra JK (2013) Familial cardiological and targeted genetic evaluation: low yield in sudden unexplained death and high yield in unexplained cardiac arrest syndromes. Hear Rhythm 10:1653-60

23. Behr ER, Dalageorgou C, Christiansen M, Syrris $P$, Hughes $S$, Tome Esteban MT, Rowland E, Jeffery S, McKenna WJ (2008) Sudden arrhythmic death syndrome: Familial evaluation identifies inheritable heart disease in the majority of families. Eur Heart ] 29:1670-1680

24. Adler A, van der Werf C, Postema PG, et al (2012) The phenomenon of "QT stunning": The abnormal QT prolongation provoked by standing persists even as the heart rate returns to normal in patients with long QT syndrome. Hear Rhythm 9:901-908

25. Sangwatanaroj S, Prechawat S, Sunsaneewitayakul B, Sitthisook S, Tosukhowong P, Tungsanga K (2001) New electrocardiographic leads and the procainamide test for the detection of the Brugada sign in sudden unexplained death syndrome survivors and their relatives. Eur Heart ] 22:2290-6

26. Priori SG, Wilde AA, Horie M, et al (2013) HRS/EHRA/APHRS Expert Consensus Statement on the Diagnosis and Management of Patients with Inherited Primary Arrhythmia Syndromes. Hear Rhythm 10:19321963

27. Heart Rhythm UK Familial Sudden Death Syndromes Statement Development Group (2008) Clinical indications for genetic testing in familial sudden cardiac death syndromes: an HRUK position statement. Heart 94:502-7

28. Behr E, Wood DA, Wright M, Syrris P, Sheppard MN, Casey A, Davies MJ, McKenna W (2003) Cardiological assessment of first-degree relatives in sudden arrhythmic death syndrome. Lancet 362:1457-1459 



\title{
Chapter

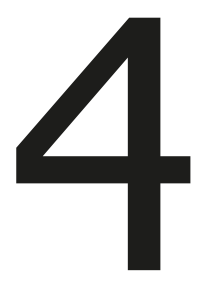

\section{Incidence and predictors of implantable cardioverter-defibrillator therapy and its complications in idiopathic ventricular fibrillation patients.}

\author{
L.J. Blom ${ }^{1}$, M. Visser ${ }^{1}$, I. Christiaans ${ }^{2}$, M.F. Scholten ${ }^{3}$,M. Bootsma ${ }^{4}$, M.P. \\ van den Berg ${ }^{5}$, S-C Yap ${ }^{6}$, J.F. van der Heijden ${ }^{1}$, P.A. Doevendans ${ }^{1,7,8}$, \\ P. Loh ${ }^{1}$, P.G. Postema ${ }^{9}$, D.Q. Barge-Schaapsveld ${ }^{10}$, N. Hofman ${ }^{2}$, P.G.A. \\ Volders $^{11}$, A.A. Wilde ${ }^{9}$, R.J. Hassink ${ }^{1}$
}

1 Department of Cardiology, University Medical Center Utrecht, Utrecht, The Netherlands 2 Department of Cardiogenetics, AMC, Amsterdam, The Netherlands

3 Department of Cardiology, Thoraxcentrum Twente, Medisch Spectrum Twente, Enschede, The Netherlands

4 Department of Cardiology, Leiden University Medical Center, Leiden, The Netherlands 5 Department of Cardiology, University Medical Center Groningen, Groningen, the Netherlands 6 Department of Cardiology, University Medical Center Rotterdam, Rotterdam, The Netherlands 7 Central Military Hospital, Utrecht, The Netherlands

8 Netherlands Heart Institute (ICIN), Utrecht, The Netherlands

9 Department of Clinical and Experimental Cardiology, Heart Centre, Amsterdam UMC, Amsterdam, The Netherlands

10 Department of Clinical Genetics, Leiden University Medical Center, Leiden, The Netherlands 11 Department of Cardiology, Cardiovascular Research Institute Maastricht (CARIM), Maastricht University Medical Centre, Maastricht, The Netherlands 


\section{ABSTRACT}

\section{Aims}

Idiopathic ventricular fibrillation (IVF) is a rare cause of sudden cardiac arrest. Implantable cardioverter-defibrillator (ICD) implantation is currently the only treatment option. Limited data are available on the prevalence and complications of ICD therapy in these patients. We sought to investigate ICD therapy and its complications in patients with IVF.

\section{Methods and results}

Patients were selected from a national registry of IVF patients. Patients in whom no underlying diagnosis was found during follow-up were eligible for inclusion. Recurrence of ventricular arrhythmia (VA) was derived from medical and ICD records, electrogram records of ICD therapies were used to differentiate between appropriate or inappropriate interventions. Independent predictors for appropriate ICD shock were calculated using cox regression. In 217 IVF patients, recurrence of sustained VAs occurred in 66 patients (30\%) during a median follow-up period of 6.1 years. Ten patients died (4.6\%). Thirty-eight patients (17.5\%) experienced inappropriate ICD therapy, and 32 patients (14.7\%) had device-related complications. Symptoms before cardiac arrest [hazard ratio (HR): 2.51, $95 \%$ confidence interval (CI): 1.48-4.24], signs of conduction disease (HR: $2.27,95 \% \mathrm{CI}: 1.15-4.47)$, and carrier of the DPP6 risk haplotype (HR: 3.24, 1.70-6.17) were identified as independent predictors of appropriate shock occurrence.

\section{Conclusion}

Implantable cardioverter-defibrillator therapy is an effective treatment in IVF, treating recurrences of potentially lethal VAs in approximately onethird of patients during long-term follow-up. However, device-related complications and inappropriate shocks were also frequent. We found significant predictors for appropriate ICD therapy. This may imply that these patients require additional management to prevent recurrent events. 
Incidence and predictors of ICD therapy and its complications in IVF | 53

\section{INTRODUCTION}

Idiopathic ventricular fibrillation (IVF) is diagnosed in patients who experienced a cardiac arrest, preferably with documented ventricular fibrillation (VF), in whom comprehensive clinical evaluation could not identify an underlying cause. ${ }^{1}$ Nowadays, an implantable cardioverterdefibrillator (ICD) remains the recommended therapy for the management of recurrent ventricular arrhythmias (VAs) in patients with IVF. ${ }^{2}$ Although some patients may benefit from pharmacological treatment with antiarrhythmic drugs or ablation of ventricular premature beats (VPBs) that trigger $\mathrm{VF}^{3-5}$ these treatments cannot replace ICD implantation. The recurrence rate of VAs in IVF patients is $17-31 \%^{6-8}$ and is highest in the first years after the index event. ${ }^{7}$ The burden of inappropriate shocks in IVF patients is $14-44 \% .^{7-10}$ So far, limited predictors of appropriate ICD therapy have been identified in IVF patients. A recent study showed that an early repolarization pattern (ERP) was the only predictor for arrhythmia recurrence. ${ }^{9}$ However, since 2013 sudden cardiac arrest victims who demonstrate ERP on their baseline electrocardiogram (ECG) are diagnosed with early repolarization syndrome (ERS) as a separate disease entity, and are therefore no longer considered idiopathic. No further studies have been conducted to identify predictors for arrhythmia recurrence in IVF patients.

In this multicentre study, we included IVF patients who had received an ICD for secondary prevention of life-threatening arrhythmias. The goal of this study was to assess the incidence of ICD therapy, complications of ICD implantation, and predictors of appropriate ICD therapy during longterm follow-up.

\section{METHODS}

\section{Patient population}

Patients were selected from a Dutch registry of IVF patients (Figure 1). This registry comprises patients from the Academic Medical Center Amsterdam (AMC), Leiden University Medical Center (LUMC), Erasmus 


\section{4 | CHAPTER 4}

Medical Center Rotterdam (EMC), University Medical Center Groningen (UMCG), Maastricht University Medical Center (MUMC), Medisch Spectrum Twente (MST), and University Medical Center Utrecht (UMCU). Data from the UMCU were previously published. ${ }^{7}$ All study parameters were retrospectively and prospectively collected. Patients were eligible for enrolment if they had experienced a cardiac arrest, with an initial shockable rhythm, and were diagnosed with IVF at time of the index event. Idiopathic ventricular fibrillation was diagnosed according to the 2013 consensus criteria. ${ }^{1}$ Idiopathic ventricular fibrillation was defined as a resuscitated cardiac arrest victim, with documented VF and/or shockable rhythm, in whom known cardiac, respiratory, metabolic, and toxicological aetiologies were excluded through clinical evaluation. Diagnostic criteria used to exclude specific diseases are shown in Table 1. The study was in accordance with all principles outlined in the declaration of Helsinki. This study was approved by the local ethics committee of the UMCU and at each enrolling centre. Due to the descriptive nature of the study, informed consent of patients was regarded unnecessary by the ethics committee.

\begin{tabular}{|c|c|c|}
\hline Diagnosis & Tests used for exclusion & Criteria used \\
\hline Coronary heart disease & $\begin{array}{l}\text { electrocardiogram, blood } \\
\text { chemistry, coronary CT/ } \\
\text { angiography }\end{array}$ & Roffi et al ${ }^{19}$ \\
\hline Coronary artery spasm & ergonovine provocation & Montalescot et $\mathrm{al}^{20}$ \\
\hline Long QT syndrome & $\begin{array}{l}\text { electrocardiogram, exercise stress } \\
\text { test, DNA analysis }\end{array}$ & Schwartz et $\mathrm{al}^{21}$ \\
\hline Brugada syndrome & $\begin{array}{l}\text { electrocardiogram, sodium } \\
\text { channel blocker provocation, DNA } \\
\text { analysis }\end{array}$ & Antzelevitch et $\mathrm{al}^{22}$ \\
\hline $\begin{array}{l}\text { Catecholaminergic } \\
\text { polymorphic ventricular } \\
\text { tachycardia }\end{array}$ & $\begin{array}{l}\text { electrocardiogram, Holter, } \\
\text { exercise stress test, DNA analysis }\end{array}$ & Priori et al $^{1}$ \\
\hline Short QT syndrome & electrocardiogram, DNA analysis & Mazzanti et al ${ }^{23}$ \\
\hline $\begin{array}{l}\text { Early repolarization } \\
\text { syndrome }\end{array}$ & electrocardiogram & Macfarlane et al ${ }^{24}$ \\
\hline $\begin{array}{l}\text { Hypertrophic } \\
\text { cardiomyopathy }\end{array}$ & Cardiac imaging, DNA analysis & Elliot et $\mathrm{al}^{25}$ \\
\hline
\end{tabular}


Incidence and predictors of ICD therapy and its complications in IVF | 55

\begin{tabular}{|lll|}
\hline Dilated cardiomyopathy & Cardiac imaging, DNA analysis & Pinto et al ${ }^{26}$ \\
\hline $\begin{array}{l}\text { Arrhythmogenic } \\
\text { cardiomyopathy }\end{array}$ & $\begin{array}{l}\text { ECG, Holter, Cardiac imaging, } \\
\text { endomyocardial biopsy, DNA } \\
\text { analysis }\end{array}$ & Marcus et al ${ }^{27}$ \\
\hline Myocarditis & $\begin{array}{l}\text { Cardiac imaging, blood chemistry, } \\
\text { endomyocardial biopsy }\end{array}$ & $\begin{array}{l}\text { Caforio et al }{ }^{28}, \\
\text { Friedrich et al }{ }^{29}\end{array}$ \\
\hline
\end{tabular}

Table 1 Diagnostic criteria and tests used for exclusion of specific diseases. ECG: Electrocardiogram; CT: Computer Tomography.

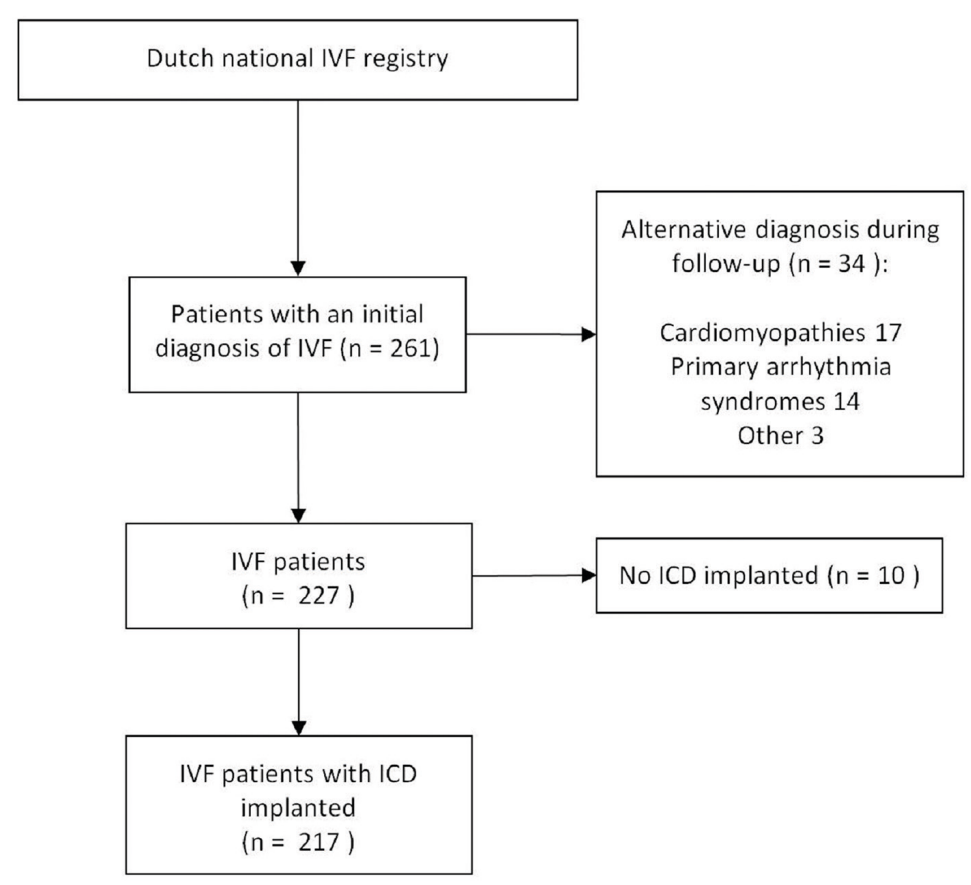

Figure 1 Patient selection. The figure shows a flow chart of the patient selection for the study population. ICD implantable cardioverterdefibrillator; IVF idiopathic ventricular fibrillation.

\section{Clinical investigation}

Enrolled patients underwent a detailed investigation of the medical history and physical examination, 12-lead ECG, laboratory test, exercise stress test, echocardiography, and coronary angiography (or CT angiography 


\section{6 | CHAPTER 4}

in patients under 40 years old). A majority of patients underwent an magnetic resonance imaging (MRI) with late gadolinium enhancement to exclude structural abnormalities. Additional evaluation was performed in patients with phenotypical clues for a specific underlying disease. In a subset of patients sodium channel blocker provocation was performed with either ajmaline (maximum dosage $1 \mathrm{mg} / \mathrm{kg}$ ), flecainide (maximum dosage $2 \mathrm{mg} / \mathrm{kg}$ ), or procainamide (maximum dosage $1000 \mathrm{mg}$ ) to exclude Brugada syndrome. Provocation test with either ergonovine or acetylcholine was performed to exclude coronary artery spasm in a subgroup of patients. Endomyocardial biopsy was performed in a subgroup of patients to evaluate signs of cardiomyopathy, myocarditis, or sarcoidosis. Toxicological screening was performed in patients with an indication of toxic or illicit drug use and consisted of a screening of cocaine, cannabis, benzodiazepines, amphetamines, and barbiturates. Electrophysiologic study was performed at the clinician's discretion, using local stimulation protocols.

DNA analysis was performed in most patients, specifically in patients suspected of concealed underlying arrhythmic disease. Genetic testing consisted of single targeted gene testing by Sanger sequencing based on phenotype detection after clinical evaluation showed clues for a specific disease, or next generation sequencing of a larger panel of genes (either 34 genes, 48, 50,63, or 212) depending on the centre where the genetic testing was performed. The tested genes were all associated with an increased risk of cardiomyopathy, VAs and sudden cardiac death (SCD), including the DPP6 risk haplotype associated with short-coupled Torsade de Pointes/IVF in Dutch patients. ${ }^{11}$ Patients with the DPP6 risk haplotype were considered as IVF patients according to the Dutch consensus. ${ }^{12}$ All minor abnormalities that were detected during clinical investigations, for example mild mitral valve regurgitation or a mildly decreased serum potassium $(2.5-3.5 \mathrm{mmol} / \mathrm{L})$, that were not sufficient to diagnose an underlying disease resulting in VF were accepted as comorbidities.

\section{Arrhythmia recurrence during follow-up}

To assess VA recurrence, we searched all available medical records 
and the ICD database of the participating centres. Wherever available, electrogram records of ICD therapies were used to determine whether they were appropriate. In other cases, we used the interpretation of the treating clinician. Appropriate therapy was defined as shocks delivered for VAs. Conversely, inappropriate therapy was defined as those delivered in the absence of VAs. Due to the retrospective nature of our analysis, ICDs of various developmental stages were included. Implantable cardioverter-defibrillator programming varied over time according to available recommendations and was adjusted during follow-up on the basis of individual clinical history and to avoid recurrences of inappropriate interventions.

\section{Statistical analysis}

We presented continuous variables as mean \pm standard deviation (SD) or median $\mathrm{p}$ inter-quartile ranges (IQR) and discrete data as frequencies and percentage. We estimated event-free survival by the Kaplan-Meier method and compared differences by log-rank tests. We analysed predictors of appropriate ICD therapy in univariate and multivariate Cox proportional hazards regression models. We selected candidate factors which we considered as potential predictors for appropriate ICD therapy based on literature, as well as factors that reached a significance level $<0.15$ in univariate analysis and contained complete data for multivariate analysis (e.g. not all investigations were performed in all patients, therefore the results from Holter registration and MRI were not included in the multivariate analysis). Logistic regression was performed for inappropriate ICD therapy data with the following variables: History of atrial fibrillation, age, sex, conduction disease, and time of ICD implantation. Data were analysed using $\mathrm{R}$ version 3.4.1 (R Foundation for Statistical Computing, Vienna, Austria). A P-value $<0.05$ was considered significant.

\section{RESULTS}

Between 1988 and 2018, we identified a total of 261 patients with an initial diagnosis of IVF. During follow-up 34 patients (13\%) were 


\section{8 | CHAPTER 4}

diagnosed with an underlying diagnosis. Ten patients (4\%) either refused ICD implantation, or were diagnosed with IVF before ICDs were routinely implanted. Hence, these patients were excluded from further analysis. Our final cohort consisted of 217 IVF patients who had an ICD implanted for secondary prevention.

\section{Baseline characteristics}

The clinical baseline characteristics of the study cohort are presented in Table 2. The final patient cohort consisted of 217 IVF patients $(60 \%$ male). The median age at time of the index event was 39 (IQR 28-52) years. Most patients experienced the event at rest (61\%). Twenty-nine patients $(13 \%)$ received a subcutaneous ICD.

\begin{tabular}{|c|c|}
\hline Patients & $n(\%)$ \\
\hline Male sex (\%) & $131(60 \%)$ \\
\hline Median age at event (years) & 39 (IQR 28-52) \\
\hline \multicolumn{2}{|l|}{ Circumstances during occurrence of VF (\%) } \\
\hline Exercise & $44(20 \%)$ \\
\hline Emotions & $11(5 \%)$ \\
\hline Rest & $132(61 \%)$ \\
\hline Sleep & $30(14 \%)$ \\
\hline \multicolumn{2}{|l|}{ Symptoms before cardiac arrest (\%) } \\
\hline Any symptoms ${ }^{\dagger}$ & $75(35 \%)$ \\
\hline Palpitations & $28(13 \%)$ \\
\hline Syncope & $27(12 \%)$ \\
\hline Family history of sudden cardiac death* (\%) & $18(8 \%)$ \\
\hline
\end{tabular}

Table 2. Baseline characteristics of 217 IVF patients. *History of SCD defined as 1 first degree family member with SCD $<45$ years, or multiple second degree family members with SCD. †Any symptoms defined 
as chest-pain, syncope, near-syncope or palpitations. IQR: interquartile range.

\section{Clinical investigation}

Table 3 shows an overview of all performed diagnostic tests. Electrocardiogram parameters of the 217 IVF patients are shown in Table 4. Blood chemistry revealed minor abnormalities in 28 patients (14\%): hypokalaemia in 22 patients, hypomagnesemia in 3 patients, and both in 3 patients. Echocardiography revealed minor abnormalities, such as mild ventricular hypertrophy or dilatation, valve prolapse or hypokinesia, in $61 / 208$ patients (29\%) in whom echocardiography was performed. Holter or telemetry monitoring was performed in $121 / 217$ patients (56\%) and revealed NSVT in 8 patients (7\%). Exercise stress testing showed multiple VPBs in 31 patients (18\%) without meeting criteria for catecholaminergic polymorphic ventricular tachycardia (VT). Coronary angiography or CT angiography was normal in all examined patients. Magnetic resonance imaging was performed in 148/217 patients (68\%), and showed minor abnormalities or focal delayed enhancement ( $<1$ segment) in $28 \%$. Sodium channel blocker provocation was performed in 130/217 (60\%) and was normal in all examined patients. Ergonovine provocation was performed and was normal in 53/217 patients (24\%). Endomyocardial biopsy revealed non-specific fibrosis or hypertrophy in one-third of examined patients (11/33; performed in $15 \%$ of total). Toxicological screening was performed in only $16 / 217$ patients (7\%), as there was no indication of toxic or illicit drug use in most cases, and revealed use of cannabis in one patient and use of benzodiazepines in one other patient. Electrophysiological study was performed in $111 / 217$ patients (51\%). Ventricular fibrillation or VT was induced in $31 / 111$ patients (28\%). Catheter ablation was performed in $32 / 111$ patients (29\%) with as main indication ablation of VPB (21 patients) or supraventricular arrhythmia (11 patients). 


\begin{tabular}{|lrr|}
\hline Diagnostic test & $n$ & $(\%)$ \\
12-lead ECG & 217 & $(100 \%)$ \\
Cardiac Imaging & 217 & $(100 \%)$ \\
Echocardiography & 208 & $(96 \%)$ \\
MRI & 148 & $(68 \%)$ \\
Coronary angiography or CT angiography & 203 & $(94 \%)$ \\
Blood chemistry & 203 & $(94 \%)$ \\
Toxicological screening & 16 & $(7 \%)$ \\
Exercise stress test & 172 & $(79 \%)$ \\
Holter monitoring & 121 & $(56 \%)$ \\
Sodium channel blocker provocation & 130 & $(60 \%)$ \\
Ergonovine provocation & 53 & $(24 \%)$ \\
DNA analysis & 176 & $(81 \%)$ \\
Electrophysiological study & 111 & $(51 \%)$ \\
Endomyocardial biopsy & 33 & $(15 \%)$ \\
\hline
\end{tabular}

\section{Table 3. Diagnostic tests performed in 217 IVF patients}

\begin{tabular}{|lrl|}
\hline Parameter & $n(\%)$ or mean/median as appropriate \\
Heart rate, bpm & 71 (SD 14) \\
PR interval, ms & 153 (IQR 140-170) \\
QRS duration, ms & 95 (IQR 86-104) \\
QTc interval, ms & 412 (IQR 397-435) \\
Atrial fibrillation & 8 (4\%) \\
Left bundle branch block & 13 (6\%) \\
Right bundle branch block & 5 (2\%) \\
Terminal activation delay* & $3 \quad(1 \%)$ \\
Fractionation $\dagger$ & $14 \quad(7 \%)$ \\
\hline
\end{tabular}

\section{Table 4. Baseline electrocardiographic parameters of 217 IVF}

patients. *Defined $\geq 55 \mathrm{~ms}$ measured from the nadir of the $S$ wave to the end of the QRS complex in V1. tdefined as the presence of various $R S R$ ' patterns (QRS duration <120 ms) with or without $Q$ wave, which include an additional $R$ wave ( $R$ prime) or notching of the $R$ wave or notching of 
the $S$ wave, or the presence of more than two $R$ primes (fragmentation) in two contiguous leads corresponding to a major lead set(anterior, lateral, inferoposterior). bpm: beats per minute, ms: milliseconds.

DNA analysis was performed in 176/217 (81\%). We performed single genetic testing based on phenotypic clues in $65(37 \%)$ patients and (for some patients: additional) screening of a gene panel in 111 (63\%) patients. In 61 (28\%) patients a variant was found. The Dutch DPP6risk haplotype was detected in 23 patients (11\%). In the other 38 patients (18\%) a variant of uncertain clinical significance (VUS) was detected (5x RyR2, 5x ANK2, 5x TTN, 3x JUP, 3x DSP, 3x SCN5A, 2x CACNA1C, 2x KCNE1, 2x KCNH2, 2x ABCC9, 2x SCN4B, ACAP9, CSRP3, DES, DPP6, DSC2, DSG2, KCNA5, KCNJ5, KCNQ1, MYH7, MYPN, SLMAP, TCAP, TMEM43). Table 5 shows the VUS detected in patients who received appropriate ICD therapy.

\begin{tabular}{|c|c|c|c|c|}
\hline Patient & Gene & VUS & & $\begin{array}{l}\text { Family history of } \\
S C D\end{array}$ \\
\hline 1 & JUP & c. $286 \mathrm{G}>\mathrm{A}$ & p.(Glu96Lys) & - \\
\hline 2 & DSP & c. $8482 \mathrm{G}>\mathrm{A}$ & p.(Gly2828Ser) & - \\
\hline \multirow[t]{2}{*}{3} & KCNE1 & c. $200 \mathrm{G}>\mathrm{A}$ & p.(Arg67His) & - \\
\hline & $\mathrm{KCNH} 2$ & c. $2717 \mathrm{C}>\mathrm{T}$ & p.(Ser906Leu) & \\
\hline 4 & KCNQ1 & c. $1193 A>G$ & p.(Lys398Arg) & - \\
\hline 6 & ANK2 & c. $9679 A>C$ & p.(Thr3227Pro) & - \\
\hline 7 & TTN & c. $4583 \mathrm{G}>\mathrm{A}$ & p.(trp1528.) & Positive \\
\hline 8 & CACNA1C & c. $1112 \mathrm{~A}>\mathrm{C}$ & p.(Tyr371Ser) & Positive \\
\hline \multirow[t]{2}{*}{9} & SCN5A & c. $3094 \mathrm{G}>\mathrm{A}$ & p.(Glu1032Lys) & - \\
\hline & RyR2 & c. $4747 C>T$ & p.(Pro1583Ser) & \\
\hline 11 & DPP6 & c. $821 \mathrm{G}>\mathrm{A}$ & p.(Arg274His) & Positive \\
\hline 12 & TCAP & c. $208 C>T$ & p.(Arg70Trp) & - \\
\hline
\end{tabular}

Table 5. Variants of uncertain significance found in patients with appropriate ICD therapy.

\section{Follow-up}

During a median follow-up period of 6.1 (2.4-12.1) years, 10 patients died ( 1 by heart failure, 6 by a non-cardiac cause, and 3 by an unknown 
cause). The overall incidence rate of appropriate ICD therapy was 5.3 per 100 person-years [95\% confidence interval (CI) 4.0-6.7].

\section{Recurrence of ventricular arrhythmia during follow-up}

In total, $64 / 217$ (29.5\%) patients received a total of 408 shocks during 164 shock episodes. Two patients (1\%) received anti-tachycardia pacing. The median time from ICD implantation to first appropriate shock was 1.1 years (IQR 0.4-2.4). The underlying arrhythmia was retrieved in 150/164 episodes (91.5\%). Ventricular fibrillation was the underlying rhythm in $105 / 164$ (64\%) episodes, followed by VT in 37/164 (22.6\%) and both VT and VF in $8 / 164(4.9 \%)$. The median number of shocks per person was 4 (IQR 2-8). Thirty-nine (18\%) patients suffered multiple shock episodes. Twenty-four patients (11.1\%) experienced an electrical storm (defined as $>3$ appropriate shocks per $24 \mathrm{~h}$ ).

\section{Inappropriate implantable cardioverter-defibrillator therapy}

Inappropriate ICD therapy was delivered in $38 / 217$ patients (17.5\%). Patients who experienced inappropriate ICD therapy had a longer follow-up duration ( 9.9 years vs. 5.6 years) and more often had their ICD implanted before 2000 (groups divided by the median calendar year of ICD implantation). Logistic regression analysis did not identify any significant factors associated with inappropriate ICD therapy. Inappropriate discharge was due to atrial fibrillation in 13 patients (34\%), supraventricular tachycardia in 8 patients (21\%), T-wave/noise oversensing, ICD or lead malfunction in 7 patients $(18 \%)$, and sinus tachycardia in 5 patients (13\%).

\section{Complications of implantable cardioverter-defibrillator}

Implantable cardioverter-defibrillator complications were seen in 32/ $217(14.7 \%)$. The most common complications were lead related (14 patients), including lead fracture, oversensing or an increase in impedance requiring lead revision. One patient had the lead (RIATA) recalled and replaced, no other known lead problems were found. Four patients had 
Incidence and predictors of ICD therapy and its complications in IVF | 63

device endocarditis, of whom one required antibiotics, two required lead extraction, and one died as a result of Staphylococcus aureus sepsis. Four patients suffered from pocket haematoma or discomfort requiring pocket revision.

\section{Predictors of implantable cardioverter-defibrillator therapy}

Table 6 shows the predictors for appropriate ICD therapy. In univariate analysis, a family history of SCD (HR: 2.90, 95\% CI: $1.57-5.34, \mathrm{P}=$ $0.0006)$ and VPBs or NSVT at Holter or telemetry monitoring (HR: 2.44, 95\% CI: $1.11-5.34, \mathrm{P}=0.026$ ) were associated with appropriate ICD therapy. Because Holter or telemetry monitoring was not performed in all patients, we did not include them in the multivariate analysis. Multivariate analysis identified three independent predictors for appropriate ICD therapy: Symptoms before cardiac arrest (HR: 2.51, 95\% CI: 1.48-4.24, $\mathrm{P}=0.0006)$, signs of conduction disease (HR: $2.27,95 \% \mathrm{CI}: 1.15-4.47$, $\mathrm{P}=0.018$ ), and carrier of the DPP6 risk haplotype (HR: $3.24,95 \% \mathrm{CI}$ :

$1.70-6.17, \mathrm{P}=0.0003)$. Figure 2 shows the observed rate of appropriate ICD therapy for the respective groups. 


\section{4 | CHAPTER 4}

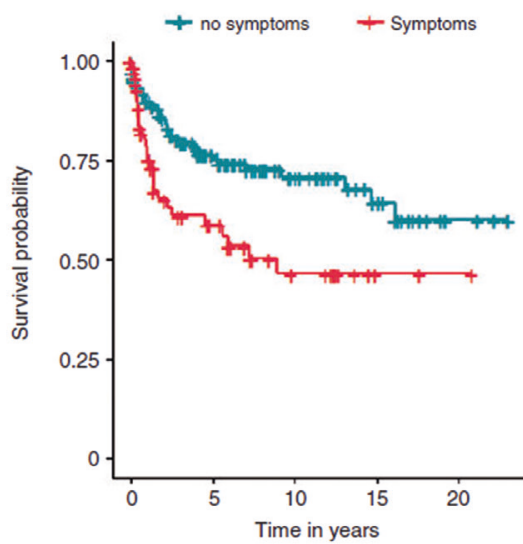

Number at risk

$\begin{array}{ccccc}142 & 67 & 34 & 17 & 4 \\ 75 & 23 & 11 & 2 & 1\end{array}$

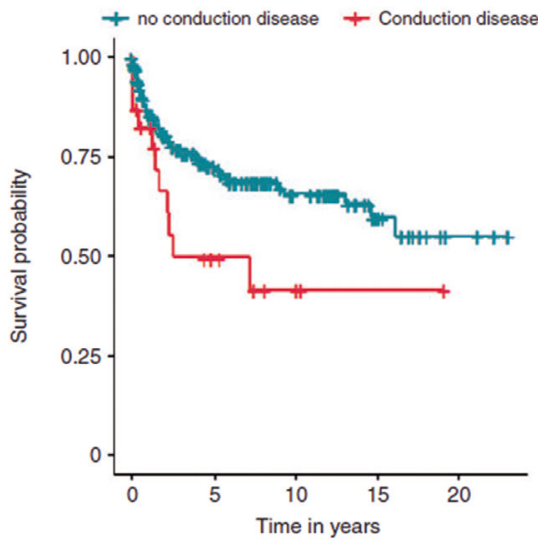

Number at risk

$\begin{array}{lllll}185 & 77 & 38 & 15 & 4\end{array}$

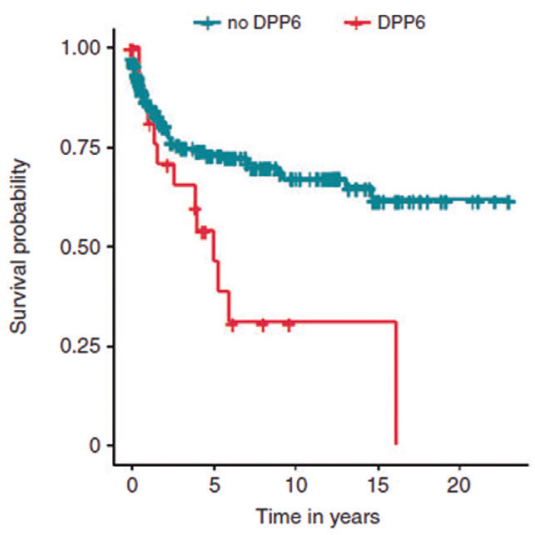

Number at risk

$\begin{array}{ccccc}194 & 83 & 44 & 18 & 5 \\ 23 & 7 & 1 & 1 & 0\end{array}$

Figure 2 Cumulative event-free survival by predictor for appropriate ICD therapy. ICD implantable cardioverter-defibrillator. 
Incidence and predictors of ICD therapy and its complications in IVF | 65

\begin{tabular}{|c|c|c|c|c|}
\hline Predictor & Univariate analysis & & $\begin{array}{l}\text { Multivariate } \\
\text { analysis }\end{array}$ & \\
\hline Baseline characteristics & HR (95\% Cl for HR) & $p$ value & $\begin{array}{l}H R(95 \% \mathrm{Cl} \\
\text { for HR) }\end{array}$ & $p$ value \\
\hline Male sex & $1.15(0.70-1.92)$ & 0.578 & & \\
\hline $\begin{array}{l}\text { Age at index event, } \\
\text { years }\end{array}$ & $1.00(0.98-1.01)$ & 0.735 & & \\
\hline $\begin{array}{l}\text { Symptoms before } \\
\text { cardiac arrest }\end{array}$ & $2.05(1.24-3.37)$ & 0.0048 & $\begin{array}{l}2.51(1.48- \\
4.24)\end{array}$ & 0.0006 \\
\hline Family history of SCD & $2.90(1.57-5.34)$ & 0.0006 & & \\
\hline Syncope & $1.58(0.75-3.33)$ & 0.225 & & \\
\hline $\begin{array}{l}\text { Index event during } \\
\text { exercise }\end{array}$ & $0.85(0.47-1.52)$ & 0.583 & & \\
\hline \multicolumn{5}{|l|}{ Baseline ECG parameters } \\
\hline Heart rate, bpm & $1.01(0.99-1.03)$ & 0.295 & & \\
\hline Conduction disease & $2.05(1.07-3.93)$ & 0.032 & $\begin{array}{l}2.27(1.15- \\
4.47)\end{array}$ & 0.018 \\
\hline Atrial fibrillation & $0.87(0.213-3.58)$ & 0.852 & & \\
\hline QTc interval, ms & $1.00(0.99-1.01)$ & 0.779 & & \\
\hline \multicolumn{5}{|l|}{ Other parameters } \\
\hline Holter: NSVT or VPBs & $2.44(1.11-5.34)$ & 0.026 & & \\
\hline $\begin{array}{l}\text { Exercise test: NSVT or } \\
\text { VPBs }\end{array}$ & $0.74(0.33-1.65)$ & 0.461 & & \\
\hline $\begin{array}{l}\text { MRI: delayed enhance- } \\
\text { ment }\end{array}$ & $1.16(0.45-2.96)$ & 0.762 & & \\
\hline VF induced during EPS & $1.23(0.56-2.72)$ & 0.09 & & \\
\hline DNA analysis: any VUS & $1.51(0.83-2.74)$ & 0.174 & & \\
\hline $\begin{array}{l}\text { DNA analysis: DPP6 } \\
\text { haplotype }\end{array}$ & $2.39(1.29-4.40)$ & 0.0054 & $\begin{array}{l}3.24(1.70- \\
6.17)\end{array}$ & 0.0003 \\
\hline
\end{tabular}

Table 6. Predictors of appropriate ICD therapy in 217 IVF patients. HR: Hazard ratio, CI: Confidence interval, Conduction disease: PR-interval > 200ms or QRS-duration $>120 \mathrm{~ms}$, ms: milliseconds, bpm: beats per minute, EPS: electrophysiological study, NSVT: non-sustained ventricular tachycardia, SCD: Sudden cardiac death, VF: ventricular fibrillation, VPB: ventricular premature beat, VUS: variant of uncertain significance. 


\section{DISCUSSION}

\section{Main findings}

This multicentre study extends the findings of our previous monocentre study in 107 patients initially diagnosed with IVF.7 We described the longterm follow-up of 217 patients with a diagnosis of IVF, after we excluded 34 patients in whom a specific diagnosis was detected during followup and 10 patients without an ICD. One-third of patients experienced recurrence of VAs during a median follow-up of 6 years. The median number of shocks was four per person. Of the patients who experienced appropriate ICD therapy, many experienced an electrical storm. We identified three predictors for recurrence of VAs: the presence of symptoms before cardiac arrest, signs of conduction disease on baseline ECG, and carrier of the DPP6 risk haplotype. Inappropriate ICD therapy was delivered in $19 \%$ of patients and was most commonly caused by atrial fibrillation. Implantable cardioverter-defibrillator complications other than inappropriate ICD therapy occurred in $15.5 \%$.

\section{Previous studies}

The Cardiac Arrest Survivors with Preserved Ejection Fraction Registry showed high rates of appropriate ICD therapy, with $16 \%$ (25 patients) who received appropriate ICD therapy and 16\% (25 patients) who received appropriate anti-tachycardia pacing. ${ }^{8}$ Previous studies showed varying rates of appropriate ICD therapy between $11 \%$ and $43 \% .{ }^{6,8,9}$ The limited data on inappropriate ICD therapy show a high rate with inappropriate shocks in $14-44 \%$ of patients. $6,8,9$ Our results are in line with these previous studies. Only one study has been published that assessed predictors for appropriate ICD therapy in IVF. ${ }^{9}$ This study by Siebermair et al., with a long-term follow-up of 35 IVF patients, identified ERP as the only predictor for the recurrence of VAs. Other independent predictors of recurrence of VAs than ERP were not identified (male sex, age at index event, atrial fibrillation and inducibility of VF/VT at electrophysiological study). With the recognition of ERS as a separate disease entity, ERP is no longer found in the IVF population. The independent predictors found 
in our study have not been analysed previously. Atrial fibrillation was the strongest predictor of inappropriate ICD therapy, which was comparable to our study as the cause of inappropriate ICD therapy was atrial fibrillation in most patients. We did not identify any significant factors associated with inappropriate ICD therapy.

\section{Significant predictors}

In a multivariate Cox proportional hazards model, we identified three independent significant predictors for appropriate ICD therapy. The first is symptoms before cardiac arrest, defined as syncope, near-syncope, chest pain, or palpitations at any time before the initial event. While nonspecific in the general population, these symptoms may all be caused by recurrent self-terminating episodes of VA. Frequent ventricular ectopy may trigger recurrent VT/VF and concomitant ICD therapy. Identifying the ectopic origin during an electrophysiologic study and targeted ablation of triggering VPB should be considered, as this has been shown to reduce the recurrence of VF in IVF patients. ${ }^{4}$

The second predictor for appropriate ICD therapy is signs of conduction disease on baseline ECG, which may result from conduction delay in the atrium, atrioventricular node, and/or His-Purkinje system. In the general population, conduction disease is associated with an increased risk of $A F$, pacemaker implantation, and all-cause mortality. ${ }^{13}$ Though the relationship between conduction disease and mortality in the absence of underlying cardiomyopathy is not clear, it is often attributed to increased fibrosis of the cardiac conduction system. ${ }^{14}$ Fibrosis and resulting conduction delay have been associated with cardiac arrhythmias ${ }^{15}$ and are common in structural heart disease as well as in the Brugada syndrome (especially in those with SCN5A mutations). ${ }^{16}$ In aging myocardium, the increased fibrosis enhances arrhythmia vulnerability and is associated with decreased expression of connexin43 and cardiac sodium channels (Nav1.5). ${ }^{17}$ Thus, both structural and ion-channel differences may explain the contribution of conduction disease to VA recurrence. However, more research is needed to identify the underlying mechanisms.

The third predictor is being carrier of the DPP6 risk haplotype. This genetic 
mutation is unique to a Dutch population. Patients in whom the DPP6 haplotype is detected are not diagnosed with a specific disease, because a clear phenotype is absent and the pathophysiological mechanism for arrhythmic risk of the mutation is not fully understood. However, it is clear the DPP6 haplotype increases arrhythmic risk. More research is needed to further identify the underlying mechanism for arrhythmia occurrence in these patients.

In univariate analysis, we identified another two predictors for appropriate ICD therapy: A family history of SCD and VPBs or NSVTs recorded at Holter or telemetry monitoring recording. A specific mechanism that may cause IVF is through short-coupled VPBs. The short-coupled VPBs can directly elicit VF. A family history of SCD indicates inherited disease. A large part of primary electrical syndromes have been found to be associated with pathological (ion-channel) mutations that are the underlying cause for VAs. ${ }^{1}$ Though a causative mutation has not been found in all IVF patients with a family history of SCD, the presence of such an undiscovered mutation in these families is plausible. Due to the rare, heterogeneous nature of IVF and the incomplete penetrance and variable expression of genetic mutations in family members, uncovering these genetic mutations remains a challenge.

\section{Management options}

Aside from ICD implantation for secondary prevention of SCD, several other management options have been studied that could benefit a subgroup of IVF patients. Firstly, the effectiveness of electrophysiologic guided class $1 \mathrm{~A}$ anti-arrhythmic therapy, primarily quinidine, has been studied. Class $1 A$ anti-arrhythmic therapy was associated with absence of VAs during long-term follow-up. ${ }^{3}$ Secondly, short-coupled VPBs have been shown to be an excellent target for catheter ablation. After ablation of these premature ventricular beats, most patients are free of recurrence of VAs. ${ }^{4,5}$ Finally, ICD implantation for primary prevention of IVF in asymptomatic family members has been performed, but its benefit is still under debate. Ten Sande et al. ${ }^{11}$ show the results of ICD implantation in asymptomatic carriers of the DPP6 risk haplotype where $<3 \%$ of family 
members received appropriate therapy during 5-year follow-up. Thus, IVF patients may benefit from a more tailored management approach. Based on our findings, we recommend thorough family and genetic screening in those patients with a positive family history for SCD and/or known DPP6 mutation carriers, regular evaluation of patients with conduction disease during follow-up for signs of underlying structural heart disease and considering ablation of VPBs in patients with recurrent ventricular ectopy.

\section{Study limitations}

A limitation of our study is the lack of systematic assessment of IVF patients. Due to the retrospective, observational nature of our cohort, not all patients underwent the diagnostic tests required to appropriately diagnose IVF. This is a common problem in IVF studies in a real-world setting ${ }^{8,9,18}$ and makes absence of underlying disease less certain. In particular, MRI (62\%) and sodium channel blocker provocation (57\%) were not routinely performed, which is comparable to other IVF cohorts, $77 \%$ and $66 \%$ in CASPER ${ }^{8}$ and $37 \%$ and $34 \%$ in Siebermair et al. ${ }^{9}$, respectively. In our current cohort, physicians are informed on the missing diagnostic tests in their patients and encouraged to complete the clinical evaluation.

Secondly, although we included a number of patients comparable to the Cardiac Arrest Survivors with Preserved Ejection Fraction Registry, the number of included patients is modest compared with large-scale ICD trials, which can be expected in any study on IVF since it is a rare disease. Lastly, ICD therapy is dependent on device programming, which was not systematically performed, possibly resulting in a higher rate of both appropriate and inappropriate ICD therapy.

\section{CONCLUSIONS}

We report the long-term follow-up of 217 IVF patients and showed that one-third of the patients experience appropriate ICD therapy, supporting the guidelines for implantation of ICD for secondary prevention in idiopathic VF patients. Inappropriate ICD therapy and ICD complications 


\section{0 | CHAPTER 4}

such as lead dysfunction were frequent problems. After multivariate analysis, we identified three independent predictors for appropriate ICD therapy: symptoms before cardiac arrest, signs of conduction disease on baseline ECG, and carrier of the DPP6 risk haplotype. After univariate analysis, the presence of VPB or NSVT on Holter or telemetry monitoring was also a predictor for VA recurrence. Idiopathic ventricular fibrillation patients with one or multiple of these factors require extra attention to prevent appropriate ICD therapy. 
Incidence and predictors of ICD therapy and its complications in IVF | 71

\section{REFERENCES}

1. Priori SG, Wilde AA, Horie M, Cho Y, Behr ER, Berul C et al. HRS/EHRA/ APHRS expert consensus statement on the diagnosis and management of patients with inherited primary arrhythmia syndromes. Europace 2013;15: 1389-406.

2. Priori SG, Blomström-Lundqvist C, Mazzanti A, Blom N, Borggrefe M, Camm J et al. 2015 ESC Guidelines for the management of patients with ventricular arrhythmias and the prevention of sudden cardiac death. Europace 2015;17:1601-87.

3. Belhassen B, Viskin S, Fish R, Glick A, Setbon I, Eldar M. Effects of electrophysiologic-guided therapy with Class IA antiarrhythmic drugs on the long-term outcome of patients with idiopathic ventricular fibrillation with or without the Brugada syndrome. J Cardiovasc Electrophysiol 1999;10:1301-12.

4. Knecht S, Sacher F, Wright M, Hocini M, Nogami A, Arentz T et al. Long-term follow-up of idiopathic ventricular fibrillation ablation. $A$ multicenter study. J Am Coll Cardiol 2009;54:522-8.

5. Haïssaguerre M, Shoda M, Jaı"s P, Nogami A, Shah DC, Kautzner J et al. Mapping and ablation of idiopathic ventricular fibrillation. Circulation 2002;106:962-7.

6. Ozaydin M, Moazzami K, Kalantarian S, Lee H, Mansour M, Ruskin JN. Long-term outcome of patients with idiopathic ventricular fibrillation: a meta-analysis. J Cardiovasc Electrophysiol 2015;26:1095-104.

7. Visser M, van der Heijden JF, van der Smagt JJ, Doevendans PA, Wilde $A A$, Loh $P$ et al. Long-term outcome of patients initially diagnosed with idiopathic ventricular fibrillation. Circ Arrhythmia Electrophysiol 2016;9:e004258.

8. Herman ARM, Cheung C, Gerull B, Simpson CS, Birnie DH, Klein GJ et al. Outcome of apparently unexplained cardiac arrest: results from investigation and follow-up of the prospective cardiac arrest survivors with preserved ejection fraction registry. Circ Arrhythm Electrophysiol 2016;9:e003619.

9. Siebermair J, Sinner M, Beckmann B-M, Laubender R, Martens E, 
Sattler $\mathrm{S}$ et al. Early repolarization pattern is the strongest predictor of arrhythmia recurrence in patients with idiopathic ventricular fibrillation: results from a single centre long-term follow-up over 20 years+. Europace 2016;18:718-25.

10. Champagne J, Geelen P, Philippon F, Brugada P. Recurrent cardiac events in patients with idiopathic ventricular fibrillation, excluding patients with the Brugada syndrome. BMC Med 2005;3:1.

11. Ten Sande JN, Postema PG, Boekholdt SM, Tan HL, Van Der Heijden JF, De Groot NMS et al. Detailed characterization of familial idiopathic ventricular fibrillation linked to the DPP6 locus. Heart Rhythm 2016;13:905-12.

12. Visser M, van der Heijden JF, Doevendans PA, Loh P, Wilde AA, Hassink RJ. Idiopathic ventricular fibrillation. Circ Arrhythmia Electrophysiol 2016;9:e003817.

13. Cheng S, Keyes MJ, Larson MG, Elizabeth L, Newton-Cheh C, Levy D et al. Long-term outcomes in individuals with a prolonged PR interval or first-degree atrioventricular block. JAMA 2009;301:2571-7.

14. Kwok CS, Rashid M, Beynon R, Barker D, Patwala A, Morley-Davies $A$ et al. Prolonged PR interval, first-degree heart block and adverse cardiovascular outcomes. Heart 2016;102:672-80.

15. De Jong S, Van Veen TAB, Van Rijen HVM, De Bakker J. Fibrosis and cardiac arrhythmias. J Cardiovasc Pharmacol 2011;57:630-8.

16. Smits JPP, Eckardt L, Probst V, Bezzina CR, Schott J], Remme CA et al. Genotype-phenotype relationship in Brugada syndrome: electrocardiographic features differentiate SCN5A-related patients from non-SCN5A-related patients. J Am Coll Cardiol 2002;40:350-6.

17. Stein $M$, Noorman $M$, van Veen TAB, Herold E, Engelen MA, Boulaksil $M$ et al. Dominant arrhythmia vulnerability of the right ventricle in senescent mice. Heart Rhythm 2008;5:438-48.

18. Waldmann V, Bougouin W, Karam N, Dumas F, Sharifzadehgan A, Gandjbakhch E et al. Characteristics and clinical assessment of unexplained sudden cardiac arrest in the real-world setting: focus on idiopathic ventricular fibrillation. Eur Heart J 2018;39:1981-7.

19. Roffi M, Patrono C, Collet J-P, Mueller C, Valgimigli M, Andreotti F, 
et al. 2015 ESC Guidelines for the management of acute coronary syndromes in patients presenting without persistent ST-segment elevation. Eur Heart J. 2016;37(3):267-315.

20. Montalescot G, Sechtem U, Achenbach S, Andreotti F, Arden C, Budaj A, et al. 2013 ESC guidelines on the management of stable coronary artery disease. Eur Heart J. 2013;34(38):2949-3003.

21. Schwartz PJ, Ackerman MJ. The long QT syndrome. Eur Heart J. 2013;34(40):3109-16.

22. Antzelevitch C, Yan G-X. J-wave syndromes: Brugada and early repolarization syndromes. Antzelevitch C, Yan G-X, editors. Heart Rhythm 2015;12(8):1852-66.

23. Mazzanti A, Kanthan A, Monteforte N, Memmi M, Bloise R, Novelli V, et al. Novel insight into the natural history of short QT syndrome. J Am Coll Cardiol. 2014;63(13):1300-8.

24. MacFarlane PW, Antzelevitch $C$, Haissaguerre M, Huikuri H V., Potse $M$, Rosso $R$, et al. The early repolarization pattern. J Am Coll Cardiol. 2015;66(4):470-7.

25. Elliott PM, Uk C, Anastasakis A, Germany MAB, Germany MB, et al. 2014 ESC Guidelines on diagnosis and management of hypertrophic cardiomyopathy. Eur Heart J. 2014;35(39):2733-79.

26. Pinto YM, Elliott PM, Arbustini E, Adler Y, Anastasakis A, Böhm M, et al. Proposal for a revised definition of dilated cardiomyopathy, hypokinetic non-dilated cardiomyopathy, and its implications for clinical practice. Eur Heart J. 2016;37(23):1850-8.

27. Marcus FI, McKenna WJ, Sherrill D, Basso C, Bauce B, Bluemke DA, et al. Diagnosis of arrhythmogenic right ventricular cardiomyopathy/ Dysplasia. Circulation 2010;121(13):1533-41.

28. Caforio ALP, Pankuweit S, Arbustini E, Basso C, Gimeno-Blanes J, Felix SB, et al. Current state of knowledge on aetiology, diagnosis, management, and therapy of myocarditis. Eur Heart J. 2013;34(33):2636-48.

29. Friedrich MG, Sechtem U, Schulz-Menger J, Holmvang G, Alakija P, Cooper LT, et al. Cardiovascular Magnetic Resonance in Myocarditis. J Am Coll Cardiol. 2009;53(17):1475-87. 



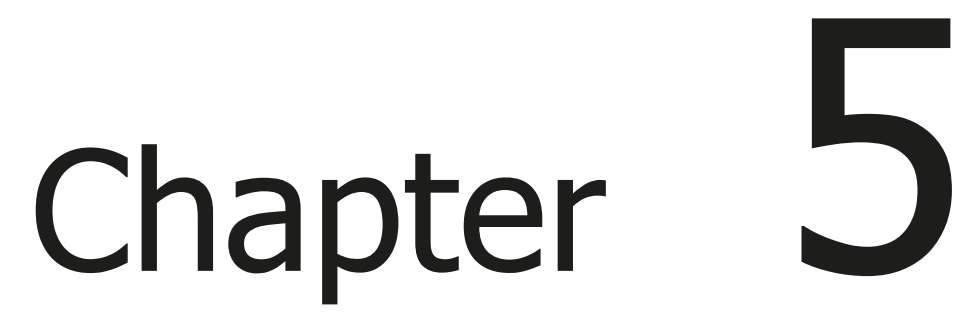

\section{Familial cascade screening in idiopathic ventricular fibrillation}

G Mellor ${ }^{1,2 *}$, LJ Blom ${ }^{3 *}$, SA Groeneveld ${ }^{3}$, B Winkel4 ${ }^{4}$ B Ensam ${ }^{1}$, C Scrocco1, J Tfelt-Hansen ${ }^{4}$, AD Krahn ${ }^{5}$, RJ Hassink ${ }^{3}$, ER Behr ${ }^{1}$

1 Cardiology Clinical Academic Group, St. George's, University of London, UK

2 Cardiology Department, Royal Papworth Hospital, Cambridge, UK

3 Department of Cardiology, University Medical Centre, Utrecht, The Netherlands

4 Department of Cardiology, Rigshospitalet, Copenhagen, Denmark

5 Division of Cardiology, University of British Columbia, Vancouver, BC, Canada

* These authors contributed equally to this work

In preparation 


\section{6 |CHAPTER 5}

\section{ABSTRACT}

\section{Introduction}

Familial cascade screening is well established in patients with heritable cardiac disease and in cases of Sudden Arrhythmic Death Syndrome (SADS). The assessment of family members of survivors of idiopathic ventricular fibrillation is not established.

\section{Methods}

Patients with idiopathic VF were identified from 4 national and institutional registries. All patients underwent comprehensive clinical evaluation to exclude other causes of cardiac arrest. The results of cascade screening in first-degree relatives were then reported.

\section{Results}

We identified 189 first-degree relatives of 89 IVF survivors. All relatives had a 12-lead resting ECG. Echocardiography was performed in 149 (78\%). At least 1 additional investigation was performed in 170 (90\%). 5 (3\%) individuals from 4 (4\%) families had diagnostic tests with positive results. Two relatives were found to carry the DPP6 risk haplotype identified in the familial proband. In three separate families the asymptomatic parent of the proband developed a type I Brugada pattern on ECG during ajmaline provocation testing.

\section{Conclusions}

We conclude that comprehensive clinical evaluation of the unexplained cardiac arrest survivor is the cornerstone of management not only of the proband but of the wider family. In cases where comprehensive clinical evaluation, supplemented by genetic testing where appropriate, has excluded heritable phenotypes, the yield of family screening is low. 


\section{INTRODUCTION}

Cardiac arrest due to ventricular fibrillation is a common presentation and important cause of death. ${ }^{1}$ The majority of cases are due to ischaemic heart disease. In other cases, cardiomyopathy or ion channel disease may be evident. In a significant minority initial investigation with ECG, transthoracic echocardiogram and coronary imaging will be nondiagnostic. ${ }^{2}$ Systematic clinical investigation of such 'unexplained cardiac arrest' (UCA) ${ }^{3}$ may reveal a diagnosis in approximately one third of cases. ${ }^{4}$ Frequently, the diagnosis made will be an inherited arrhythmia syndrome or cardiomyopathy which will lead to a recommendation to perform cascade screening of immediate family members. ${ }^{5}$

If no diagnosis is made, a label of idiopathic ventricular fibrillation (IVF) is used although there is no consensus on which investigations are required before IVF is diagnosed.

Therefore, those patients labelled with IVF may represent a variety of underlying pathologies including: concealed forms of established arrhythmia syndromes or cardiomyopathies; short-coupled VF without documentation of the short-coupled PVC; or undiagnosed environmental triggers in otherwise normal hearts. The benefit of family cascade screening in such cases is not known.

Since incomplete penetrance and variable expressivity are well recognised in the inherited arrhythmia syndromes, we hypothesized that familial screening may reveal diagnoses in first-degree relatives that were not apparent in the IVF survivor. We present a cohort of idiopathic VF survivors where family cascade screening has been undertaken. Details of the investigations performed and the overall diagnostic yield is discussed.

\section{METHODS}

Cases of unexplained cardiac arrest were identified from four large national and institutional registries in Canada, Denmark, the Netherlands and the UK. Individuals were included in the study if they had been resuscitated from cardiac arrest with documentation of ventricular 


\section{8 |CHAPTER 5}

fibrillation (VF) and no diagnosis had been made after exhaustive clinical assessment. Diagnoses of Early Repolarization Syndrome (ERS) and short-coupled Ventricular Fibrillation (SC-VF) were considered part of the spectrum of idiopathic VF and were not excluded.

All individuals were required to have undergone a minimum clinical assessment comprising at least: resting 12-lead ECG; cardiac imaging with echocardiogram and/or MRI; coronary artery imaging; exercise ECG, and; sodium channel blocker provocation. Further investigations were performed at the treating physician's discretion. Genetic testing was not required for inclusion although results were recorded where performed. In addition, minor or non-diagnostic findings from clinical investigations were recorded. A summary of the minimum required clinical assessment and acceptable minor findings is shown in table 1 . 
FAMILIAL CASCADE SCREENING IN IVF| 79

\begin{tabular}{|ll|}
\hline Clinical Investigation & Non-significant findings \\
\hline ECG* & QTc $\leq 460 \mathrm{~ms}$ ( $\leq 480 \mathrm{~ms}$ for females) \\
& $\begin{array}{l}\text { Infero-lateral early repolarisation pattern } \\
\text { Partial right bundle branch block with acute } r^{\prime} \text { beta angle }\end{array}$ \\
\hline Echocardiogram** & LVEF $\geq 50 \%$ \\
& Absence of significant regional wall motion abnormalities \\
\hline $\begin{array}{l}\text { Cardiac magnetic reso- } \\
\text { nance imaging }\end{array}$ & LVEF $\geq 50 \%$ \\
\hline Coronary imaging & Late gadolinium enhancement at RV insertion \\
\hline Exercise stress test & Isolated monomorphic PVCs. \\
\hline $\begin{array}{l}\text { Na-channel blocker } \\
\text { provocation }\end{array}$ & ST segment depression with normal coronaries \\
\hline
\end{tabular}

\section{Table 1. Minimum clinical assessment required for inclusion}

of proband. All listed investigations must be considered normal for inclusion. Minor findings as described were acceptable. ECG:

Electrocardiogram, LVEF: left ventricular ejection fraction, PVC: premature ventricular contraction, RV: right ventricle. *Allowing for temporary (2 weeks) peri-arrest repolarization changes. **Allowing for temporary 'myocardial stunning' post-arrest ( 2 weeks)

First-degree relatives of IVF survivors who have been assessed with at least a resting 12 lead ECG were identified. Demographic data of relatives were collected. Results of the ECGs and additional investigations performed in relatives were recorded and coded as: normal, abnormal or borderline. The diagnosis of an inherited arrhythmia syndrome was noted and associations with proband and relatives characteristics were sought.

\section{Statistical Methods}

The Mann-Whitney $U$ test was used to compare continuous variables. Fisher's exact test was used to compare categorical variables. A $p<0.05$ was considered significant. Statistical analysis was performed with SPSS 24 (SPSS, Inc., Chicago, IL). 


\section{RESULTS}

We included 89 families of IVF probands where cascade screening with at least a 12-lead ECG was performed. The final cohort comprised 189 firstdegree relatives of these 89 idiopathic VF survivors.

\section{Proband characteristics}

The clinical characteristics of probands are summarised in table 2. The mean age was $38.5 \pm 12$ years and $48(54 \%)$ were male. The circumstance of cardiac arrest was recorded in 74 (83\%). The majority occurred at rest (39, 53\%). Prior suspected arrhythmic syncope was reported in 17 (19\%). A prior familial history of sudden death was reported in 12 (13\%). In addition to the required investigations, an ECG with high right ventricular leads was performed in 81 (91\%), signal-averaged ECG (SAECG) in 41 (47\%), cardiac MRI in 75 (83\%) and adrenaline provocation in 31 (36\%).

\begin{tabular}{|c|c|c|c|c|}
\hline Characteristic, n (\%) & $\begin{array}{l}\text { All } \\
\text { probands } \\
(n=89)\end{array}$ & $\begin{array}{l}\text { Negative } \\
\text { family } \\
\text { screening } \\
(n=85)\end{array}$ & $\begin{array}{l}\text { Positive } \\
\text { family } \\
\text { screening } \\
(n=4)\end{array}$ & $\begin{array}{l}p \text {-val- } \\
\text { ue }\end{array}$ \\
\hline Age, mean \pm SD & $38.5 \pm 12$ & $38.9 \pm 12$ & $28 \pm 17$ & NS \\
\hline Male Sex & $48(54)$ & $45(54)$ & $3(75 \%)$ & NS \\
\hline \multicolumn{5}{|c|}{ Circumstance of cardiac arrest } \\
\hline Sleep & $11(15)$ & $9(13)$ & $2(50)$ & \\
\hline Rest & $39(53)$ & $38(55)$ & $1(25)$ & NS \\
\hline Exercise & $24(32)$ & $23(32)$ & $1(25)$ & \\
\hline Prior syncope & $17(19)$ & $16(19)$ & $1(25)$ & NS \\
\hline Other FH SD (present) & $12(13)$ & $12(14)$ & $0(0)$ & NS \\
\hline \multicolumn{5}{|l|}{ Investigations } \\
\hline ECG $(n=89,100 \%)$ & & & & \\
\hline
\end{tabular}


FAMILIAL CASCADE SCREENING IN IVF | 81

\begin{tabular}{|c|c|c|c|c|}
\hline Normal & $57(63)$ & $54(63)$ & $3(75)$ & \multirow{3}{*}{ NS } \\
\hline Early Repolarisation & $15(18)$ & $14(18)$ & $1(25)$ & \\
\hline Other Minor findings & $17(19)$ & $17(19)$ & $0(0)$ & \\
\hline \multicolumn{5}{|c|}{ High lead ECG $(n=81,91 \%)$} \\
\hline Normal & $79(98)$ & 75 (97) & $4(100)$ & \multirow{2}{*}{ NS } \\
\hline Type 2 Brugada pattern & $2(2)$ & $2(3)$ & $0(0)$ & \\
\hline \multicolumn{5}{|l|}{ SAECG $(n=41,47 \%)$} \\
\hline Normal (0/3) & $30(74)$ & $29(73)$ & $1(100)$ & \multirow{3}{*}{ NS } \\
\hline Abnormal $(\geq 2 / 3)$ & $6(14)$ & $6(15)$ & $0(0)$ & \\
\hline Equivocal $(1 / 3)$ & $5(12)$ & $5(12)$ & $0(0)$ & \\
\hline \multicolumn{5}{|l|}{ Echo $(n=89,100 \%)$} \\
\hline Normal & $72(81)$ & $69(81)$ & $3(75)$ & \multirow{2}{*}{ NS } \\
\hline Minor finding & 17 (19) & $16(19)$ & $1(25)$ & \\
\hline \multicolumn{5}{|l|}{ CMR $(n=75,83 \%)$} \\
\hline Normal & $62(83)$ & $60(85)$ & $2(50)$ & \multirow{2}{*}{ NS } \\
\hline Equivocal & $13(17)$ & $11(16)$ & $2(50)$ & \\
\hline \multicolumn{5}{|c|}{ Exercise ECG $(n=88,99 \%)$} \\
\hline Normal & $73(82)$ & $69(81)$ & $4(100)$ & \multirow{2}{*}{ NS } \\
\hline Minor findings & $15(18)$ & 15 (19) & $0(0)$ & \\
\hline \multicolumn{5}{|c|}{ Adrenaline provocation $(n=31,36 \%)$} \\
\hline Negative & $22(69)$ & $20(67)$ & $2(100)$ & \multirow{3}{*}{ NS } \\
\hline QT prolongation >30ms & $1(3)$ & $1(3)$ & $0(0)$ & \\
\hline QT prolongation 1-30ms & $8(28)$ & $8(30)$ & $0(0)$ & \\
\hline \multicolumn{5}{|c|}{ Na blocker provocation $(n=89,100 \%)$} \\
\hline Negative & $89(100)$ & $86(100)$ & $4(100)$ & \multirow{2}{*}{ NA } \\
\hline Type 1 Brugada ECG & $0(0)$ & $0(0)$ & $0(0)$ & \\
\hline \multicolumn{5}{|l|}{ Genetics $(n=66,74 \%)$} \\
\hline Normal & $44(67)$ & $43(70)$ & $1(25)$ & \multirow{3}{*}{0.04} \\
\hline VUS & $21(31)$ & $19(30)$ & $2(50)$ & \\
\hline Pathogenic variant & $1(2)$ & $0(0)$ & $1(25)$ & \\
\hline
\end{tabular}

Table 2. Clinical characteristics and summary of investigation findings in idiopathic VF survivors stratified by presence of an inherited arrhythmia syndrome diagnosis made in a first-degree 
relative. ECG: Electrocardiogram; FH SD: Family history of Sudden Cardiac Death; NA: not applicable; NS: not significant; SAECG: Signalaveraged electrocardiogram; SD: Standard deviation; VUS: variant of uncertain significance.

\section{Relative characteristics}

The clinical characteristics of relatives are displayed in table 3. The mean age was $39 \pm 20$ years and $107(56 \%)$ were male. Regarding the relationship to the proband, 56 (30\%) were parents, 66 (35\%) were siblings and $68(36 \%)$ were offspring. Prior syncope was reported in 10 (5\%). A prior familial history of sudden death (other than the proband) was reported in 7 (4\%).

As per the protocol, all relatives had a resting 12-lead ECG, with additional high right ventricular leads performed in 52 (27\%). Further investigations included: an echocardiogram in 149 (78\%), cardiac MRI in $22(12 \%)$ [22\% had no imaging], exercise ECG in 117 (62\%); SAECG in $59(31 \%)$; sodium-channel blocker challenge in 20 (11\%). An adrenaline provocation was performed in $6(3 \%)$ relatives. Genetic testing was performed in 31 (16\%).

\begin{tabular}{|lllll|}
\hline Characteristic & $\begin{array}{l}\text { All rel- } \\
\text { atives } \\
(\mathbf{n = 1 8 9})\end{array}$ & $\begin{array}{l}\text { No familial } \\
\text { diagnosis } \\
(\mathbf{n = 1 7 3 )}\end{array}$ & $\begin{array}{l}\text { Familial } \\
\text { diagnosis } \\
(\mathbf{n = 1 6 )}\end{array}$ & $\begin{array}{l}p \text {-val- } \\
\text { ue }\end{array}$ \\
\hline Age & $39 \pm 20$ & $39 \pm 20$ & $41 \pm 21$ & NS \\
Male Sex & $107(56)$ & $100(58)$ & $7(44)$ & NS \\
Relationship to proband & & & & \\
$\quad$ Child & $67(36)$ & $65(38)$ & $2(13)$ & NS \\
Sibling & $66(35)$ & $57(33)$ & $9(56)$ & \\
Parent & $56(30)$ & $51(29)$ & $5(31)$ & NS \\
Syncope & $10(5)$ & $10(6)$ & $0(0)$ & NS \\
Palpitations & $25(14)$ & $23(14)$ & $2(13)$ & NS \\
Chest pain & $15(8)$ & $15(9)$ & $0(0)$ &
\end{tabular}


FAMILIAL CASCADE SCREENING IN IVF| 83

\begin{tabular}{|c|c|c|c|c|}
\hline Shortness of breath & $1(1)$ & $1(1)$ & $0(0)$ & NS \\
\hline Other FH SD (present) & $7(4)$ & $6(3)$ & $1(6)$ & NS \\
\hline \multicolumn{5}{|l|}{ Investigations } \\
\hline \multicolumn{5}{|l|}{ ECG $(n=189,100 \%)$} \\
\hline Normal & $160(85)$ & $112(85)$ & $14(88)$ & \multirow{3}{*}{ NS } \\
\hline Early Repolarisation & $11(6)$ & $11(6)$ & $0(0)$ & \\
\hline Other Abnormal & $17(9)$ & $15(9)$ & $2(13)$ & \\
\hline \multicolumn{5}{|c|}{ High lead ECG $(n=52,27 \%)$} \\
\hline Normal & $49(94)$ & $44(98)$ & $6(75)$ & \multirow{2}{*}{ NS } \\
\hline Abnormal & $3(6)$ & $1(2)$ & $2(25)$ & \\
\hline \multicolumn{5}{|l|}{ SAECG $(n=59,31 \%)$} \\
\hline Normal & $52(88)$ & $48(91)$ & $4(67)$ & \multirow{2}{*}{ NS } \\
\hline Abnormal & $7(12)$ & $5(9)$ & $2(33)$ & \\
\hline \multicolumn{5}{|c|}{ Echocardiogram $(n=148,78 \%)$} \\
\hline Normal & $134(91)$ & $124(90)$ & $10(100)$ & \multirow{2}{*}{ NS } \\
\hline Abnormal & $14(9)$ & $14(10)$ & $0(0)$ & \\
\hline \multicolumn{5}{|l|}{ CMR $(n=22,12 \%)$} \\
\hline Normal & $17(77)$ & $16(76)$ & $1(100)$ & \multirow{2}{*}{ NS } \\
\hline Abnormal & $5(23)$ & $5(24)$ & $0(0)$ & \\
\hline \multicolumn{5}{|c|}{ Exercise ECG $(n=116,62 \%)$} \\
\hline Normal & $103(89)$ & $97(89)$ & $6(86)$ & \multirow{2}{*}{ NS } \\
\hline Abnormal & $13(11)$ & $12(11)$ & $1(14)$ & \\
\hline \multicolumn{5}{|c|}{ Adrenaline provocation $(n=5,3 \%)$} \\
\hline Negative & $5(100)$ & $5(100)$ & $0(0)$ & \multirow{2}{*}{ NA } \\
\hline Positive & $0(0)$ & $0(0)$ & $0(0)$ & \\
\hline \multicolumn{5}{|c|}{ Na blocker provocation $(n=20,11 \%)$} \\
\hline Normal & $17(85)$ & $15(100)$ & $2(40)$ & \multirow{2}{*}{0.009} \\
\hline Abnormal & $3(15)$ & $0(0)$ & $3(60)$ & \\
\hline \multicolumn{5}{|l|}{ Genetics $(n=30,16 \%)$} \\
\hline Negative & $28(94)$ & $22(100)$ & $6(75)$ & \multirow{2}{*}{ NS } \\
\hline Pathogenic variant & $2(6)$ & $0(0)$ & $2(25)$ & \\
\hline
\end{tabular}

Table 3. Clinical characteristics and summary of investigations 


\section{for relatives stratified by presence of an inherited arrhythmia} syndrome diagnosis. ECG: electrocardiogram; CMR: cardiac magnetic resonance imaging; SAECG: signal-averaged electrocardiogram; FH SD: Family history of sudden cardiac death.

\section{Yield of family screening}

Five (3\%) relatives from $4(4 \%)$ families had a positive diagnostic tests result. The DPP6 risk haplotype was identified in 2 relatives from one family following cascade genetic testing. A type I Brugada pattern ECG was induced during sodium-channel blocker provocation testing in 3 relatives from 3 unrelated families. There was no significant difference between the clinical characteristics of probands where a familial diagnosis was made in first-degree relatives compared to those where no familial diagnosis was made. Similarly, there were no significant differences between clinical characteristics of relatives in whom a familial diagnosis was made compared to those without a familial diagnosis. (See tables 2 and 3). The families with positive findings are summarized below:

\section{Family 1}

A 54 year-old male (from the Dutch registry) suffered VF during sleep. Clinical assessment revealed only a single minor criterion for ARVC with dyskinesia of the right ventricular lateral wall and a RV ejection fraction of $44 \%$. Genetic testing with a 34 gene panel identified the DPP6 risk-haplotype. Four relatives ( 2 children and two siblings) underwent cascade genetic testing. The brother and daughter were found to carry the haplotype. A primary prevention subcutaneous ICD was implanted in the daughter.

\section{Family 2}

A 21 year-old male suffered VF during sleep. He had a history of prior syncope. Clinical assessment, including Na-channel blocker provocation testing, revealed no positive findings. Three relatives ( 2 parents and 1 sibling) underwent clinical assessment. His 62 year-old father was found to have a type 2 Brugada ECG pattern in the standard and high RV lead positions at rest which converted to a type 1 pattern with ajmaline prov- 
ocation (1mg/kg over 5 mins). He also had an abnormal SAECG. He was asymptomatic. Genetic testing in both proband and father was negative. The father received lifestyle advice as per recommended guidelines. An ICD was not implanted.

Family 3

An 18 year-old male suffered VF during exercise. Clinical assessment, including Na-channel blocker provocation testing, revealed only basal septal hypokinesia seen both on an echocardiogram and CMR. Five relatives ( 2 parents and 3 siblings) underwent clinical assessment. His 49 year-old father was found to have a type 2 Brugada ECG pattern in the standard and high RV lead positions at rest which converted to a type 1 pattern with ajmaline provocation (1mg/kg over 5 mins). During exercise testing changes in RV conduction approaching a type 1 Brugada ECG pattern were also noted. Genetic testing in both proband and father was negative. The father received lifestyle advice as per recommended guidelines. An ICD was not implanted.

Family 4

A 19 year-old female suffered VF during rest. Clinical assessment, including Na-channel blocker provocation testing, revealed no positive findings. Genetic testing with a 212 gene panel revealed a variant of uncertain significance in the CACNA1C gene (c.6637G>A p.(Asp2213Asn)). Her 61 year-old mother received flecainide infusion for treatment of atrial fibrillation. During the infusion she converted to a type 1 Brugada ECG pattern. She was found to carry the same variant in the CACNA1C gene. She was asymptomatic and received lifestyle advice as per recommended guidelines. An ICD was not implanted.

\section{Early Repolarization}

An infero-lateral Early Repolarization (ER) pattern was seen in $14(16 \%)$ probands. ER was less common in relatives $(n=11,6 \%, p=0.007)$. There was no significant difference in relatives with ER between families where ER was seen in the proband compared to families where ER was not seen in the proband $(2 / 14,14 \%$ vs. $9 / 75,12 \%, p=0.66)$. In addition, 2 probands had a resting type 2 Brugada ECG pattern (intermittent in 


\section{6 |CHAPTER 5}

1) which did not convert to a type 1 pattern with Na channel blocker provocation. Brugada syndrome was not diagnosed in relatives of either family. However, ER was noted in a single relative from one family.

\section{Genetic Testing}

Genetic testing was performed in 66 (74\%) probands. Testing strategies varied from single phenotype panels to broad, multi-phenotype panels of up to 212 genes. A pathogenic finding was noted in 1 (2\%); a male proband of Dutch ancestry was found to carry the DPP6 risk haplotype. Cascade screening identified 2 positive relatives (as described above). In addition, 13 variants of uncertain significance (VUS) in ANK2, DSP, DSG2, CACNA1C, KCNQ1, ABCC9, TTN, JUP, RYR2 and DSC2 were identified in $21(24 \%)$ probands. The DSC2 variant was not found in either of the proband's parents. The CACNA1C mutation was found in the proband's mother in family 4 with positive findings. Predictive testing for the remaining VUS was not carried out.

\section{DISCUSSION}

Idiopathic VF is diagnosed in a VF survivor where no cause can be identified after comprehensive clinical evaluation. IVF is rare although the precise prevalence is unknown, in part due to a lack of consensus on what compromises a comprehensive clinical evaluation. ${ }^{6}$ Previous studies of IVF have often contained a heterogeneous cohort of patients with investigations carried out in varying depth. ${ }^{7}$ The inclusion criteria for this study are purposefully strict to exclude, as confidently as possible, recognised inherited arrhythmia syndromes and other causes of cardiac arrest. The yield of clinical familial evaluation in this well characterised cohort of IVF cases was low with only 5 (3\%) individuals from 4 (4\%) families with a positive diagnostic test result. Furthermore, those cases where a diagnostic test was positive for cardiac abnormalities are not necessarily diagnosed with an inherited disease.

The DPP6 risk haplotype is currently the only well-established genetic 
culprit associated with familial IVF. ${ }^{8}$ However, identified cases have been limited to Dutch families due to a founder mutation effect in that population. ${ }^{9}$ There have been no reports of IVF associated with the DPP6 risk haplotype without Dutch heritage. Furthermore, the role of genetic testing in idiopathic VF and unexplained cardiac arrest remains unclear. The yield of pathogenic/likely pathogenic variants is low in IVF series. ${ }^{7,10}$ In a large series of unexplained cardiac arrest survivors, the yield was $19 \%^{11}$ although a proportion of those patients did have an identifiable phenotype after comprehensive clinical assessment. This illustrates the value of initial clinical evaluation after cardiac arrest, as subtle phenotypic abnormalities are required to guide genetic testing in the idiopathic VF patient. ${ }^{12}$ It also explains the higher yield of genetic testing in SADS families, ${ }^{13,14}$ where the evaluation of an electrical phenotype (e.g. Brugada pattern on ECG) in the proband is limited.

\section{Early Repolarisation}

Similar to the original study by Haissaguerre et $\mathrm{al}_{,}{ }^{15}$ we found that the inferolateral Early Repolarisation pattern (ER) was common in idiopathic VF probands. Although ERS is now accepted as a diagnostic classification, there has been little progress in the understanding of the underlying pathophysiology over the last decade since its re-emergence as a significant ECG finding. Previous reports have demonstrated heritability of the ER pattern and it has been shown to be over-represented in relatives of sudden death victims. ${ }^{16,17}$ In keeping with this, ER was more common in families of probands displaying ER. However, no monogenic cause was identified in any of these families, again reflecting the wider literature which has not demonstrated convincing evidence of any single monogenic cause of ERS. It is therefore likely that ER represents a polygenic risk modifier for ventricular arrhythmias rather than a specific monogenic disease entity.

\section{Brugada based upon positive ajmaline}

The diagnosis of Brugada Syndrome by positive ajmaline test in asymptomatic patients is under debate ${ }^{18,19}$ due to the rate of false 
positives in healthy controls. In studies performed in SUD/UCA families there are high yields for establishing a diagnosis of Brugada Syndrome, ${ }^{20,21}$ while there is concern that confounding results may have resulted in misdiagnosis of Brugada Syndrome in these families. ${ }^{18,22}$ In the diagnosed families in our cohort, the affected probands all had negative ajmaline tests, suggesting that the test results in their family members may have been false-positives. Though the definition of false-positive sodium channel block challenge is difficult to define because of the lack of a gold standard. ${ }^{19}$ Routine usage of ajmaline testing in relatives of IVF patients, in whom Brugada Syndrome has been excluded, should therefore not be encouraged.

\section{Management of IVF families}

The evaluation of first-degree relatives in IVF families is complex and requires a multidisciplinary team and approach. ${ }^{12}$ The findings and diagnostic evaluation performed in the proband are needed to guide the extent of family screening and interpret their results. The appropriate diagnostic tests for evaluation of a family member might vary on a case to case basis. Therefore, referral to an inherited cardiovascular disease clinic should be considered in all IVF families to guide the evaluation of relatives.

\section{Limitations}

The study was a retrospective analysis and therefore may be susceptible to bias. Small cohort size limits the analysis of clinical predictors for familial diagnosis. Because of the retrospective nature of the study, standardized evaluation of family members was not performed for the vast majority of the cohort, and extend of diagnostic testing differed between individuals.

\section{CONCLUSION}

We conclude that comprehensive clinical evaluation of the unexplained cardiac arrest survivor is the cornerstone of management not only of the 
proband but of the wider family. In cases where comprehensive clinical evaluation, supplemented by genetic testing where appropriate, has excluded heritable phenotypes, the yield of family screening is low. 


\section{References}

1. Zipes DP, Wellens HJJ. Sudden Cardiac Death. Circulation 1998;98:2334-51.

2. Waldmann V, Bougouin W, Karam N, Dumas F, Sharifzadehgan A, Gandjbakhch E, et al. Characteristics and clinical assessment of unexplained sudden cardiac arrest in the real-world setting: Focus on idiopathic ventricular fibrillation. Eur Heart J 2018;39:1981-7.

3. Krahn AD, Healey JS, Chauhan V, Birnie DH, Simpson CS, Champagne J, et al. Systematic assessment of patients with unexplained cardiac arrest: Cardiac Arrest Survivors With Preserved Ejection Fraction Registry (CASPER). Circulation 2009;120:278-85.

4. Herman ARM, Cheung C, Gerull B, Simpson CS, Birnie DH, Klein GJ, et al. Outcome of Apparently Unexplained Cardiac Arrest: Results From Investigation and Follow-Up of the Prospective Cardiac Arrest Survivors With Preserved Ejection Fraction Registry. Circ Arrhythm Electrophysiol 2016;9:e003619.

5. Zipes DP, Camm a J, Borggrefe M, Buxton AE, Chaitman B, Fromer $M$, et al. ACC/AHA/ESC 2006 guidelines for management of patients with ventricular arrhythmias and the prevention of sudden cardiac death: A report of the American College of Cardiology/American Heart Association Task Force and the European Society of Cardiology Com. Europace 2006;8:746-837.

6. Visser M, Heijden JF van der, Doevendans PA, Loh P, Wilde AA, Hassink RJ. Idiopathic Ventricular Fibrillation. Circ Arrhythmia Electrophysiol 2016;9:e003817.

7. Leinonen JT, Crotti L, Djupsjöbacka A, Castelletti S, Junna N, Ghidoni $A$, et al. The genetics underlying idiopathic ventricular fibrillation: $A$ special role for catecholaminergic polymorphic ventricular tachycardia? Int J Cardiol Elsevier Ireland Ltd; 2018;250:139-45.

8. Alders M, Koopmann TT, Christiaans I, Postema PG, Beekman L, Tanck MWTT, et al. Haplotype-Sharing Analysis Implicates Chromosome 7q36 Harboring DPP6 in Familial Idiopathic Ventricular Fibrillation. Am J Hum Genet The American Society of Human Genetics; 2009;84:468-76. 
9. Postema PG, Christiaans I, Hofman N, Alders M, Koopmann TT, Bezzina $C R$, et al. Founder mutations in the Netherlands: familial idiopathic ventricular fibrillation and DPP6. Netherlands Hear ] 2011;19:290-6.

10. Visser M, Dooijes D, Smagt J] van der, Heijden JF van der, Doevendans PA, Loh $\mathrm{P}$, et al. Next-generation sequencing of a large gene panel in patients initially diagnosed with idiopathic ventricular fibrillation. Hear Rhythm 2017;14:1035-40.

11. Mellor G, Laksman ZWM, Tadros R, Roberts JD, Gerull B, Simpson CS, et al. Genetic Testing in the Evaluation of Unexplained Cardiac Arrest: From the CASPER (Cardiac Arrest Survivors with Preserved Ejection Fraction Registry). Circ Cardiovasc Genet 2017;10:1-8.

12. Priori SG, Wilde AA, Horie M, Cho Y, Behr ER, Berul C, et al. HRS/ EHRA/APHRS Expert Consensus Statement on the Diagnosis and Management of Patients with Inherited Primary Arrhythmia Syndromes. Hear Rhythm 2013;10:1932-63.

13. Kumar S, Peters S, Thompson T, Morgan N, Maccicoca I, Trainer A, et al. Familial cardiological and targeted genetic evaluation: low yield in sudden unexplained death and high yield in unexplained cardiac arrest syndromes. Hear Rhythm 2013;10:1653-60.

14. Behr ER, Dalageorgou C, Christiansen M, Syrris P, Hughes S, Tome Esteban MT, et al. Sudden arrhythmic death syndrome: Familial evaluation identifies inheritable heart disease in the majority of families. Eur Heart J 2008;29:1670-80.

15. Haïssaguerre M, Derval N, Sacher F, Jesel L, Deisenhofer I, Roy L de, et al. Sudden cardiac arrest associated with early repolarization. $N$ Engl J Med 2008;358:2016-23.

16. Nunn LM, Bhar-Amato J, Lowe MD, MacFarlane PW, Rogers P, McKenna WJ, et al. Prevalence of J-point elevation in sudden arrhythmic death syndrome families. J Am Coll Cardiol 2011;58:28690.

17. Mellor G, Nelson CP, Robb C, Raju H, Wijeyeratne Y, Hengstenberg $\mathrm{C}$, et al. The Prevalence and Significance of the Early Repolarization Pattern in Sudden Arrhythmic Death Syndrome Families. Circ Arrhythmia Electrophysiol 2016;9:1-7. 


\section{2 | CHAPTER 5}

18. Tadros R, Nannenberg EA, Lieve K V., Škorić-Milosavljević $D$, Lahrouchi N, Lekanne Deprez RH, et al. Yield and Pitfalls of Ajmaline Testing in the Evaluation of Unexplained Cardiac Arrest and Sudden Unexplained Death: Single-Center Experience With 482 Families. JACC Clin Electrophysiol 2017;3:1400-8.

19. Viskin S, Rosso R, Friedensohn L, Havakuk O, Wilde AAM. Everybody has Brugada syndrome until proven otherwise? Hear Rhythm 2015;12:1595-8.

20. Papadakis M, Papatheodorou E, Mellor G, Raju H, Bastiaenen R, Wijeyeratne $Y$, et al. The Diagnostic Yield of Brugada Syndrome After Sudden Death With Normal Autopsy. J Am Coll Cardiol 2018;71:120414.

21. Lahrouchi N, Raju H, Lodder EM, Papatheodorou E, Ware JS, Papadakis M, et al. Utility of Post-Mortem Genetic Testing in Cases of Sudden Arrhythmic Death Syndrome. J Am Coll Cardiol 2017;69:213445.

22. Cheung CC, Mellor G, Deyell MW, Ensam B, Batchvarov V, Papadakis $M$, et al. Comparison of Ajmaline and Procainamide Provocation Tests in the Diagnosis of Brugada Syndrome. JACC Clin Electrophysiol 2019;5:504-12. 
FAMILIAL CASCADE SCREENING IN IVF | 93 



\title{
Chapter
}

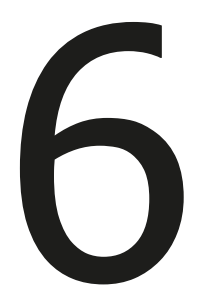

\section{Novel use of repolarization parameters in electrocardiographic imaging to uncover arrhythmogenic substrate}

\author{
L.J. Blom ${ }^{1}$, S.A. Groeneveld ${ }^{1}$, B.M. Wulterkens ${ }^{1}$,B. van Rees ${ }^{2}$, U.C. Nguyen ${ }^{2}$, \\ R.W. Roudijk ${ }^{1}$, M. Cluitmans ${ }^{2}$, P.G.A. Volders², R.J. Hassink ${ }^{1}$
}

1 Department of Cardiology, University Medical Center Utrecht, Utrecht, The Netherlands

2 Department of Cardiology, Cardiovascular Research Institute Maastricht (CARIM), Maastricht University Medical Center, Maastricht, The Netherlands

Journal of Electrocardiology - submitted 


\section{ABSTRACT}

\section{Background}

Measuring repolarization characteristics is challenging and has been reserved for experienced physicians. In electrocardiographic imaging (ECGI), activation-recovery interval (ARI) is used as a measure of local cardiac repolarization duration. We hypothesized that repolarization characteristics, such as local electrogram morphology and local and global dispersion of repolarization timing and duration could be of significance in ECGI.

\section{Objective}

To further explore their potential in arrhythmic risk stratification we investigated the use of novel repolarization parameters in ECGI.

\section{Methods}

We developed and compared methods for T-peak and T-end detection in reconstructed potentials. All methods were validated on annotated reconstructed electrograms (EGMs). Characteristics of the reconstructed EGMs and epicardial substrate maps in IVF patients were analyzed by using data recorded during sinus rhythm. The ECGI data were analyzed for EGM morphology, conduction, and repolarization.

\section{Results}

We acquired ECGI data from 8 subjects for this study. In all patients we evaluated four repolarization parameters: Repolarization time, T-wave area, Tpeak-Tend interval, and T-wave alternans. Most prominent findings were steep repolarization time gradients in regions with flat EGMs. These regions were also characterized by low $\mathrm{T}$-wave area and large differences in Tpeak-Tend interval.

\section{Conclusions}

Measuring novel repolarization parameters in reconstructed electrograms 
acquired with ECGI is feasible, can be done in a fully automated manner and may provide additional information on underlying arrhythmogenic substrate for risk stratification. Further studies are needed to investigate their potential use and clinical application. 


\section{INTRODUCTION}

Electrical signals from the heart have been vital in our understanding of underlying heart disease since the invention of the electrocardiogram (ECG) at the start of the 20th century. Since then, several invasive and non-invasive methods have been developed to record electrical heart signals and diagnose electrical heart disease or provide arrhythmic risk stratification. Interpretation of the recorded signals has proven challenging and is reserved for experienced physicians. However, due to the increased volume and complexity of the recordings, more of these tasks are automated nowadays. ${ }^{1}$

A novel method for mapping electrical activity of the heart is electrocardiographic imaging (ECGI), an emerging noninvasive highresolution imaging modality. ${ }^{2,3}$ ECGI employs a mathematical algorithm to reconstruct the electrical activity at the level of the heart muscle, from extensive (typically 64-256) body-surface ECGs and a digital, patientspecific body and heart geometry using anatomical imaging. It uses a model of the propagation of the electromagnetic field (from the heart to the body surface) to reconstruct the electrical source of the recorded body-surface ECGs. ${ }^{4}$

In ECGI, the recovery interval is used as a measure of local cardiac repolarization duration. The activation-recovery interval (ARI) is defined as the time between the steepest downslope of the QRS complex to the steepest upslope of the T wave at a single cardiac location, which is thought to reflect local action potential duration (APD). ${ }^{5}$ The potential value of other repolarization parameters in ECGI has not been studied.

We hypothesized that repolarization characteristics, such as local T wave morphology and local and global dispersion of repolarization timing and duration could be of significance in ECGI. This may especially hold for patients with idiopathic ventricular fibrillation (IVF), where the underlying substrate for ventricular arrhythmia is still unknown. However, local repolarization characteristics that contribute to arrhythmic risk ${ }^{6}$ are difficult 
to detect. We investigated several epicardial electrogram characteristics that are known to reflect dispersion of repolarization duration, a known risk factor for ventricular arrhythmias. ${ }^{7}$ On the standard 12-lead ECG, spatial dispersion of repolarization is reflected by T-wave area, ${ }^{8}$ transmural dispersion of repolarization duration by T-wave peak to T-wave end (Tpeak-Tend) interval, ${ }^{9,10}$ and temporal dispersion of repolarization duration by T-wave alternans and beat-to-beat variability of repolarization duration. ${ }^{11-13}$ To further explore their potential in arrhythmic risk stratification we investigated the use of these repolarization parameters by ECGI.

\section{METHODS}

\section{Signal acquisition}

The study protocol was approved by the Medical Research Ethics Committee of Maastricht University Medical Centre, and informed consent was obtained from all patients. We acquired body-surface potentials and a CT with electrode positions and heart-torso geometry in 8 participants. Body-surface potentials were recorded from over 200 unipolar electrodes using a dedicated ECGI system (ActiveTwo Setup, BioSemi, Amsterdam, The Netherlands) with a sampling frequency of $2048 \mathrm{~Hz}$. During acquisition, potential recordings were notch-filtered to reduce powerline interference. The body-surface signals were combined with patient-specific heart-torso geometry and processed with ECGI algorithms to reconstruct epicardial potentials as described previously. ${ }^{14}$

\section{Detection algorithms}

We developed and compared methods for T-peak and T-end detection in reconstructed potentials. For T-peak detection, we filtered the reconstructed electrograms using a 2nd order Savitzky-Golay filter (window length 199 samples). After filtering, two algorithms were applied: one based on the work of Cesari et al ${ }^{15}$ used in 12-lead ECG T-peak detection, and a newly developed method based on wave characteristics. For T-end detection, we compared two algorithms using the 'tangent 
method'16 and the 'tail method', ${ }^{17}$ respectively. All methods were validated on reconstructed electrograms (EGMs) annotated by two physicians ( $L B$ and RR). A detailed overview of the methods and validation of the detection algorithms can be found in Appendix I.

\section{Data analysis}

Characteristics of the reconstructed EGMs and epicardial substrate maps in IVF patients were analyzed by using data recorded during sinus rhythm. The ECGI data were analyzed for EGM morphology, conduction, and repolarization. The T-wave area on reconstructed epicardial unipolar EGM is defined as the area under the curve ranging from the manually determined start of the T-wave until the end of the T-wave determined by the tangent method. The Tpeak-Tend interval was measured as the time in milliseconds from the peak of the T-wave until the end of the T-wave using the developed algorithm. Recovery time was determined from the maximum positive slope of the EGM T-wave.

\section{RESULTS}

\section{Patient characteristics}

We acquired ECGI data from 7 IVF patients and one healthy subject for this study. In all patients we evaluated four repolarization parameters: Repolarization duration (RT), T-wave area, Tpeak-Tend interval, and T-wave alternans. Patient characteristics and measurements are displayed in Table 1. 
NOVEL REPOLARIZATION PARAMETERS IN ECGI| 101

\begin{tabular}{|c|c|c|c|c|c|c|c|c|}
\hline & P1 & $\mathbf{P 2}$ & P3 & P4 & P5 & C & P6 & P7 \\
\hline $\begin{array}{l}\text { Diagno- } \\
\text { sis }\end{array}$ & IVF & IVF & IVF & IVF & IVF & - & IVF & IVF \\
\hline $\begin{array}{l}\text { Age in } \\
\text { years }\end{array}$ & 57 & 54 & 42 & 23 & 47 & 50 & 73 & 24 \\
\hline Sex & $\begin{array}{l}\text { fe- } \\
\text { male }\end{array}$ & $\begin{array}{l}\text { fe- } \\
\text { male }\end{array}$ & male & $\begin{array}{l}\text { fe- } \\
\text { male }\end{array}$ & male & male & male & male \\
\hline $\begin{array}{l}\text { 12-lead } \\
\text { ECG }\end{array}$ & $\begin{array}{l}\text { flat } \\
S T / T\end{array}$ & LBBB & $\begin{array}{l}\text { nor- } \\
\text { mal }\end{array}$ & $\begin{array}{l}\text { nor- } \\
\text { mal }\end{array}$ & $\begin{array}{l}\text { nor- } \\
\text { mal }\end{array}$ & $\begin{array}{l}\text { nor- } \\
\text { mal }\end{array}$ & $\begin{array}{l}\text { nor- } \\
\text { mal }\end{array}$ & $\begin{array}{l}\text { nor- } \\
\text { mal }\end{array}$ \\
\hline \multicolumn{9}{|c|}{ Tpeak-Tend (ms) } \\
\hline $\begin{array}{l}\text { mean } \\
(\mathrm{SD}) \\
\text { range } \\
\text { (min-max) }\end{array}$ & $\begin{array}{l}60 \\
(23) \\
5-166\end{array}$ & $\begin{array}{l}71 \\
(15) \\
12- \\
167\end{array}$ & $\begin{array}{l}59 \\
(24) \\
15- \\
163\end{array}$ & $\begin{array}{l}83 \\
(45) \\
17- \\
189\end{array}$ & $\begin{array}{l}72 \\
(25) \\
28- \\
246\end{array}$ & $\begin{array}{l}75 \\
(28) \\
12- \\
195\end{array}$ & $\begin{array}{l}95 \\
(70) \\
24- \\
334\end{array}$ & $\begin{array}{l}65 \\
(38) \\
6- \\
235\end{array}$ \\
\hline \multicolumn{9}{|c|}{ T-wave area $\left(\mu \mathbf{V}^{2}\right)$} \\
\hline $\begin{array}{l}\text { mean } \\
(\mathrm{SD}) \\
\text { range } \\
\text { (min-max) }\end{array}$ & $\begin{array}{l}27 \\
(27) \\
0-173\end{array}$ & $\begin{array}{l}115 \\
(119) \\
0-587\end{array}$ & $\begin{array}{l}37 \\
(28) \\
0-189\end{array}$ & $\begin{array}{l}31 \\
(27) \\
0-181\end{array}$ & $\begin{array}{l}47 \\
(37) \\
0-272\end{array}$ & $\begin{array}{l}34 \\
(25) \\
0-142\end{array}$ & $\begin{array}{l}167 \\
(176) \\
0-957\end{array}$ & $\begin{array}{l}45 \\
(45) \\
0- \\
250\end{array}$ \\
\hline \multicolumn{9}{|l|}{ RT (ms) } \\
\hline $\begin{array}{l}\text { mean } \\
(\mathrm{SD})\end{array}$ & $\begin{array}{l}336 \\
(27)\end{array}$ & $\begin{array}{l}293 \\
(47)\end{array}$ & $\begin{array}{l}295 \\
(24)\end{array}$ & $\begin{array}{l}340 \\
(33)\end{array}$ & $\begin{array}{l}352 \\
(38)\end{array}$ & $\begin{array}{l}299 \\
(36)\end{array}$ & $\begin{array}{l}317 \\
(79)\end{array}$ & $\begin{array}{l}328 \\
(30)\end{array}$ \\
\hline $\begin{array}{l}\text { range } \\
\text { (min-max) }\end{array}$ & $\begin{array}{l}303- \\
403\end{array}$ & $\begin{array}{l}217- \\
366\end{array}$ & $\begin{array}{l}260- \\
336\end{array}$ & $\begin{array}{l}294- \\
413\end{array}$ & $\begin{array}{l}249- \\
401\end{array}$ & $\begin{array}{l}105- \\
360\end{array}$ & $\begin{array}{l}114- \\
400\end{array}$ & $\begin{array}{l}247- \\
379\end{array}$ \\
\hline
\end{tabular}

\section{Table 1. Patient characteristics and repolarization measurements}

C: Control; ECG: Electrocardiogram; IVF: Idiopathic ventricular fibrillation; LBBB Left bundle branch block; ms: milliseconds; P: Patient; RT: Recovery time; SD:

Standard deviation; 


\section{Epicardial electrocardiographic imaging data}

Representative maps of RT, T-wave area and Tpeak-Tend interval demonstrate marked local variety in repolarization characteristics in all subjects (Figure 1 and Supplemental Figures 1-6). Most prominent findings were steep repolarization time gradients in regions with flat EGMs (Figure 2). These regions were also characterized by low T-wave area and large differences in Tpeak-Tend interval. Within regions of homogenous RT, differences in T-wave area and Tpeak-Tend interval were frequent. 

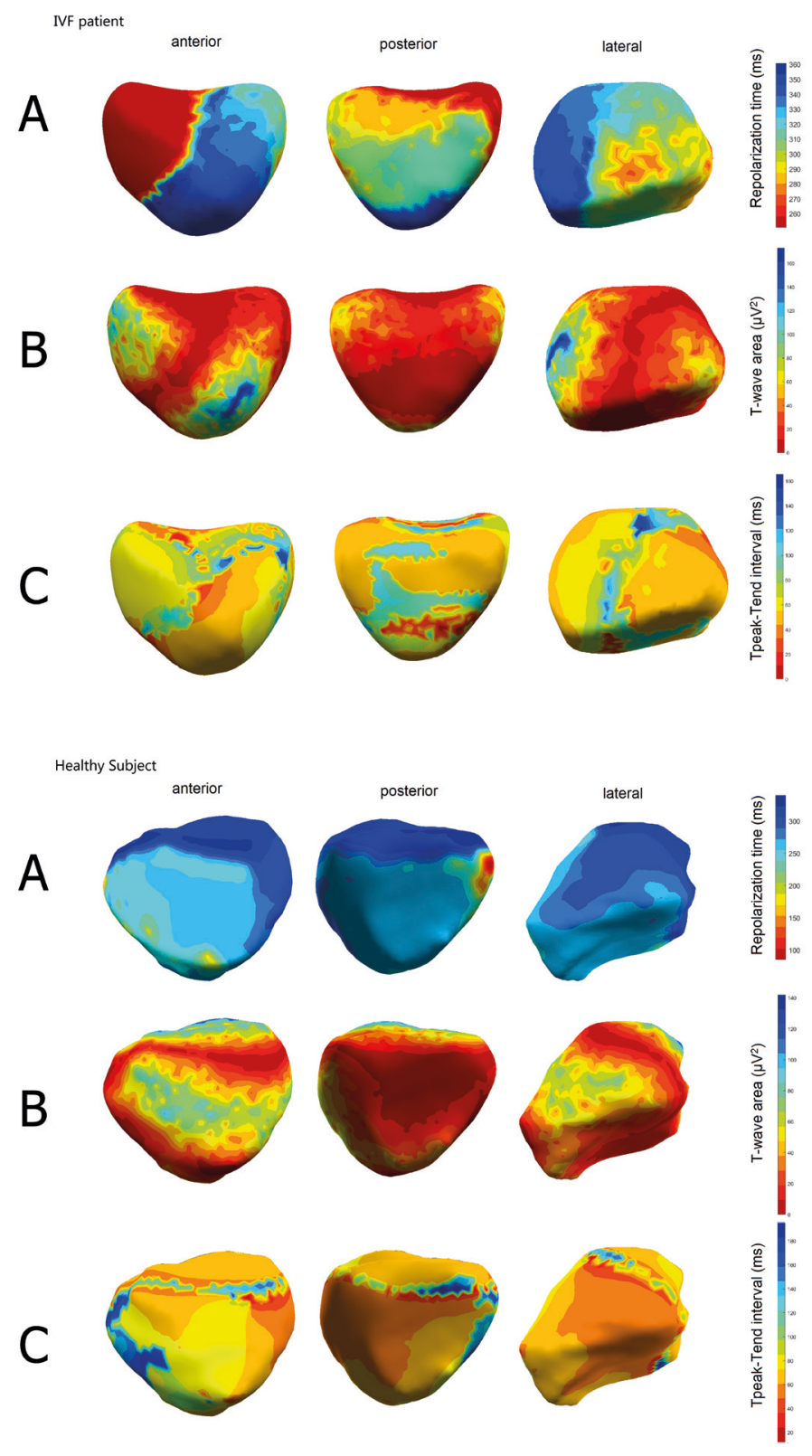

Figure 1 Epicardial maps of reconstructed electrogram repolarization time, T-peak to T-end interval and T-wave amplitude of an IVF patient and healthy subject. IVF idiopathic ventricular fibrillation; $m s$ milliseconds. 

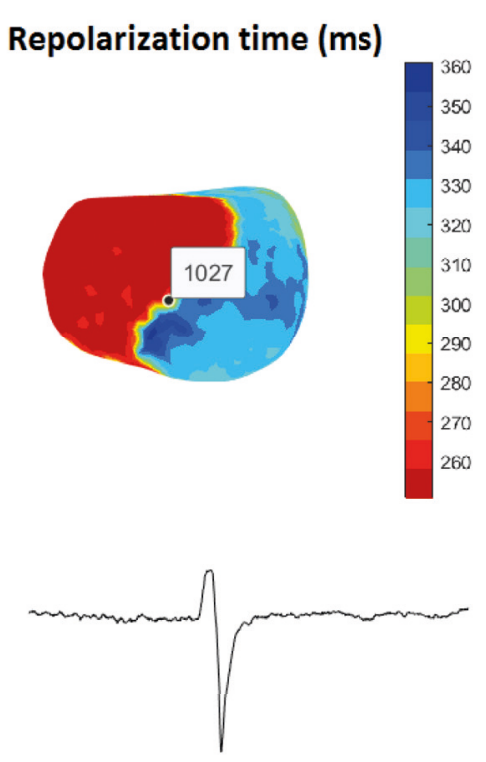

Tpeak-Tend interval (ms)
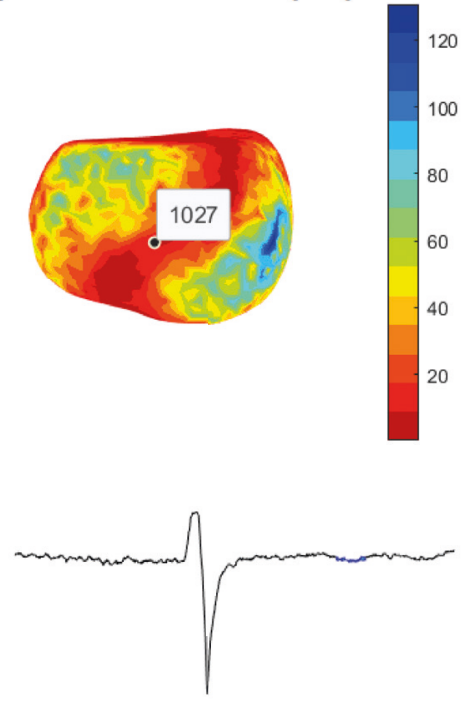

Figure 2 Reconstructed epicardial electrograms at regions of flat repolarization. Areas with areas of steep repolarization gradients (left) coincide with areas of low amplitude T-waves (right). $m s$ milliseconds

\section{T-wave area}

Figure 1B shows epicardial maps of T-wave area for an IVF patient and the healthy subject. The IVF patient has two marked areas of increased T-wave area, located at the RV free wall and LV apex respectively (arrows). Similar phenomena were seen in other IVF patients (Supplemental Figures 1-6). In the healthy subject, absolute differences in T-wave area are smaller and areas are distributed more evenly over the epicardial surface, with one large region of increased T-wave area located on the anterior epicardium.

\section{Tpeak-Tend interval}

Figure $1 \mathrm{C}$ shows the Tpeak-Tend intervals of an IVF patient and the healthy subject. Both show a heterogeneous distribution of Tpeak-Tend intervals over the epicardium with the largest regional differences in areas 
with flat EGMs. Overall, Tpeak-Tend interval distributions followed the pattern of T-wave area and RT.

\section{T-wave alternans}

Figure 3 shows the T-wave alternans of 5 consecutive beats in the healthy subject and an IVF patient. Slight beat-to-beat variation of repolarization time is seen, with most prominent differences on the anterior wall. The IVF patients show similar distributions. Most of the epicardial EGMs show no significant beat-to-beat change. 


\section{6 | CHAPTER 6}
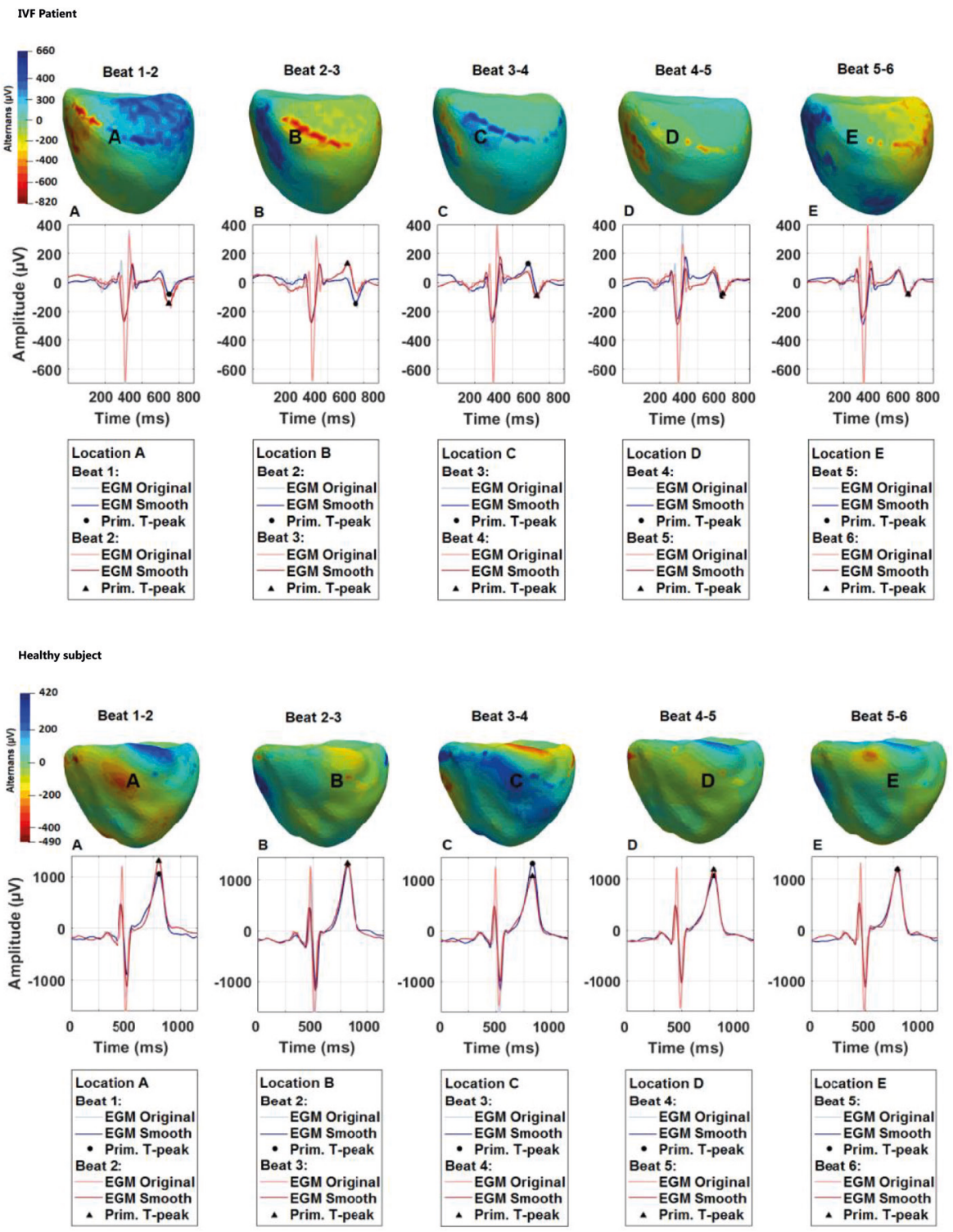

Figure 3 T-wave alternans. Epicardial potential difference maps and reconstructed electrograms for 6 consecutive beats in an IVF patient and healthy subject. EGM electrogram; ms milliseconds; prim. primary; IVF idiopathic ventricular fibrillation. 


\section{DISCUSSION}

In this study, we have shown the potential value of additional repolarization parameters in ECGI, compared to repolarization duration alone. Measuring these parameters in reconstructed electrograms is feasible, can be done in a fully automated manner and may provide additional information on morphology and dispersion of repolarization for arrhythmic risk stratification.

\section{Measuring repolarization in electrocardiographic imaging}

The reconstruction of epicardial potentials using ECGI has been validated extensively in torso-tank and canine experiments, and in human studies ${ }^{18}$. Though some studies focused on morphological correlation of EGMs, most research has been done comparing the ARI to invasively measured local action potential duration (APD). These measurements are purely based on the maximal downslope of the QRS ( $\mathrm{dV} / \mathrm{dt} \min$ ) and the latest maximal upstroke of the T-wave ( $\mathrm{dV} / \mathrm{dt}$ max). The morphological attributes of the reconstructed EGM, which provide more information on the relationship between local and remote activity or the local vector of repolarization, are not taken into account. Our results show that there are regional differences in morphology, described by Tpeak-Tend interval and T-wave area. The significance of these regional differences should be further evaluated in larger studies to assess its validity in clinical applications.

\section{Differences between ECG and ECGI repolarization parameters}

The presence of local dispersion of repolarization duration is not discernable on the 12-lead ECG. The local EGMs reconstructed with ECGI provide data to measure local activation and recovery times that approximate APD by calculating the ARI.5 The T-wave morphology of local EGMs provides data on local vectors of repolarization and differences between local and remote activity. 5,9,19,20 Therefore, repolarization abnormalities present in localized regions of the ventricles may well be uncovered by analyzing the morphology of local EGMs using ECGI. 


\section{Diagnostic potential of ECGI in primary arrhythmic substrates}

The non-invasive reconstruction of local EGMs for the identification of arrhythmic substrates has been studied extensively, ${ }^{2,21-23}$ with variable results. Most studies focusing on repolarization are specifically looking at crowding isochrones of ARI causing steep repolarization time gradients. ${ }^{24}$ Neighboring regions with large differences in repolarization time are thought to be susceptible to extrasystoles that subsequently trigger reentrant tachycardia or ventricular fibrillation. ${ }^{25}$ Differences in repolarization morphology and vector between regions have not been studied, but may contribute to arrhythmogenic substrate. In patients where conventional diagnostic tests have not been able to define an underlying substrate for ventricular arrhythmias (e.g. IVF patients), the analysis of morphological properties of local EGMs acquired through ECGI may help to uncover underlying arrhythmogenic substrate.

\section{Limitations}

Although we showed that acquisition of additional repolarization parameters from ECGI data is feasible and able to demonstrate regional differences in these parameters, this study has several important limitations. Firstly, the ability to generalize the results and draw a conclusion regarding usability of novel repolarization parameters in ECGI is limited by the relatively small number of patients. Secondly, quantitative evaluation of characteristic findings in IVF patients could not be made due to lack of a representative control population. The algorithm for automated T-peak and T-end detection has been adapted for use in ECG imaging data and requires further validation in future large scale ECGI studies.

In this study we sought to demonstrate the utility of additional repolarization parameters to the existing ECGI technique for characterizing potentially arrhythmogenic substrate. The majority of the methods used in our study have been designed and implemented as a proof of concept, and it serves as a useful guide to researchers in this field. However, these are preliminary data, and the methods will be further developed to 
integrate it in the current ECGI method. Future work includes validating the methods and characterizing repolarization in a larger cohort of IVF patients, family members and controls to identify underlying substrate for arrhythmogenic risk stratification.

\section{CONCLUSIONS}

Measuring novel repolarization parameters in reconstructed electrograms acquired with ECGI is feasible, can be done in a fully automated manner and may provide additional information on underlying arrhythmogenic substrate for risk stratification. Further studies are needed to investigate their potential use and clinical application. 


\section{REFERENCES}

1. Schläpfer J, Wellens HJ: Computer-Interpreted Electrocardiograms: Benefits and Limitations. J Am Coll Cardiol 2017; 70:1183-1192.

2. Ramanathan C, Ghanem RN, Jia P, Ryu K, Rudy Y: Noninvasive electrocardiographic imaging for cardiac electrophysiology and arrhythmia. Nat Med 2004; 10:422-428.

3. Cluitmans MJM, Peeters RLM, Westra RL, Volders PGA: Noninvasive reconstruction of cardiac electrical activity: update on current methods, applications and challenges. Netherlands Hear J 2015; 23:301-311.

4. Cluitmans MJM, Clerx M, Vandersickel N, Peeters RLM, Volders PGA, Westra RL: Physiology-based regularization of the electrocardiographic inverse problem. Med Biol Eng Comput Springer Berlin Heidelberg, 2017; 55:1353-1365.

5. Coronel R, de Bakker JMT, Wilms-Schopman FJG, Opthof T, Linnenbank AC, Belterman CN, Janse MJ: Monophasic action potentials and activation recovery intervals as measures of ventricular action potential duration: Experimental evidence to resolve some controversies. Hear Rhythm 2006; 3:1043-1050.

6. Killeen MJ, Sabir IN, Grace AA, Huang CLH: Dispersions of repolarization and ventricular arrhythmogenesis: Lessons from animal models. Prog Biophys Mol Biol Elsevier Ltd, 2008; 98:219-229.

7. Kuo CS, Atarashi H, Reddy CP, Surawicz B: Dispersion of ventricular repolarization and arrhythmia: Study of two consecutive ventricular premature complexes. Circulation 1985; 72:370-376.

8. Van Huysduynen BH, Swenne CA, Draisma HHM, Antoni ML, Van De Vooren $\mathrm{H}$, Van Der Wall EE, Schalij MJ: Validation of ECG indices of ventricular repolarization heterogeneity: A computer simulation study. J Cardiovasc Electrophysiol 2005; 16:1097-1103.

9. Opthof T, Coronel R, Wilms-Schopman FJG, Plotnikov AN, Shlapakova IN, Danilo P, Rosen MR, Janse MJ: Dispersion of repolarization in canine ventricle and the electrocardiographic $T$ wave: Tp-e interval does not reflect transmural dispersion. Hear Rhythm 2007; 4:341-348.

10. Lubinski A, Kornacewicz-Jach Z, Wnuk-Wojnar AM, Adamus J, M. K, 
Królak T, Lewicka-Nowak E, Radomski M, Swiatecka G: The Terminal Portion of the T Wave: A New Electrocardiographic Marker of Risk of Ventricular Arrhythmias. Pacing Clin Electrophysiol 2000; 23:19571959.

11. Weiss JN, Karma A, Shiferaw Y, Chen PS, Garfinkel A, Qu Z: From pulsus to pulseless: The saga of cardiac alternans. Circ Res 2006; 98:1244-1253.

12. Verrier RL, Klingenheben $T$, Malik M, El-Sherif $N$, Exner D V., Hohnloser SH, Ikeda T, Martínez JP, Narayan SM, Nieminen T, Rosenbaum DS: Microvolt T-wave alternans: Physiological basis, methods of measurement, and clinical utilityconsensus guideline by international society for Holter and noninvasive Electrocardiology. J Am Coll Cardiol 2011; 58:1309-1324.

13. Narayan SM, Franz MR, Lalani G, Kim J, Sastry A: T-Wave Alternans, Restitution of Human Action Potential Duration, and Outcome. J Am Coll Cardiol 2007; 50:2385-2392.

14. Cluitmans MJM, Bonizzi P, Karel JMH, Das M, Kietselaer BLJH, de Jong MMJ, Prinzen FW, Peeters RLM, Westra RL, Volders PGA: In Vivo Validation of Electrocardiographic Imaging. JACC Clin Electrophysiol 2017; 3:232-242.

15. Cesari M, Mehlsen J, Mehlsen A-B, Dissing Sorensen HB: Application of a new robust ECG T-wave delineation algorithm for the evaluation of the autonomic innervation of the myocardium. 2016 38th Annu Int Conf IEEE Eng Med Biol Soc IEEE, 2016, pp. 3801-3804.

16. Lepechekin E, Surawicz B: The Measurement of the Q-T Interval of the Electrocardiogram. Circulation 1952; 6:378-388.

17. Erikssen G, Liestøl K, Gullestad L, Haugaa KH, Bendz B, Amlie JP: The terminal part of the QT interval ( $T$ peak to $T$ end): A predictor of mortality after acute myocardial infarction. Ann Noninvasive Electrocardiol 2012; 17:85-94.

18. Cluitmans M, Brooks DH, MacLeod R, Dössel O, Guillem MS, Van Dam PM, Svehlikova J, He B, Sapp J, Wang L, Bear L: Validation and opportunities of electrocardiographic imaging: From technical achievements to clinical applications. Front Physiol 2018; 9:1-19. 


\section{2 | CHAPTER 6}

19. Conrath $C E$, Opthof T: Ventricular repolarization: An overview of (patho)physiology, sympathetic effects and genetic aspects. Prog Biophys Mol Biol 2006; 92:269-307.

20. Opthof $\mathrm{T}$, Coronel $\mathrm{R}$, Janse $\mathrm{MJ}$ : Is there a significant transmural gradient in repolarization time in the intact heart?: Repolarization gradients in the intact heart. Circ Arrhythmia Electrophysiol 2009; 2:89-96.

21. Vijayakumar R, Silva JNA, Desouza KA, Abraham RL, Strom M, Sacher F, Van Hare GF, Haissaguerre M, Roden DM, Rudy Y: Electrophysiologic Substrate in Congenital Long QT Syndrome: Noninvasive Mapping With Electrocardiographic Imaging (ECGI). Circulation 2014; 130:19361943.

22. Zhang J, Sacher F, Hoffmayer K, et al.: Cardiac Electrophysiological Substrate Underlying the ECG Phenotype and Electrogram Abnormalities in Brugada Syndrome Patients. Circulation 2015; 131:1950-1959.

23. Cuculich PS, Zhang J, Wang Y, Desouza KA, Vijayakumar R, Woodard PK, Rudy Y: The electrophysiological cardiac ventricular substrate in patients after myocardial infarction: Noninvasive characterization with electrocardiographic imaging. J Am Coll Cardiol 2011; 58:1893-1902.

24. Zhang J, Hocini M, Strom M, Cuculich PS, Cooper DH, Sacher F, Haïssaguerre M, Rudy Y: The Electrophysiological Substrate of Early Repolarization Syndrome: Noninvasive Mapping in Patients. JACC Clin Electrophysiol 2017; 3:894-904.

25. Benito B, Guasch E, Rivard L, Nattel S: Clinical and mechanistic issues in early repolarization: Of normal variants and lethal arrhythmia syndromes. J Am Coll Cardiol 2010; 56:1177-1186. 




\section{Chapter}

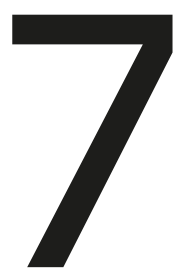

\section{Automatic triage of 12-lead electrocardiograms using deep convolutional neural networks}

R.R. van de Leur ${ }^{1}$, L.J. Blom ${ }^{1}$, E. Gavves ${ }^{2}$, I.E. Hof ${ }^{1}$, J.F. van der Heijden ${ }^{1}$, N.C. Clappers ${ }^{1}$, P.A. Doevendans ${ }^{1,3}$, R.J. Hassink ${ }^{1}$, R. van $E s^{1}$

1 Department of Cardiology, University Medical Center Utrecht, Utrecht, The Netherlands 2 QUVA Deep Vision Lab, University of Amsterdam, Amsterdam, The Netherlands 3 Netherlands Heart Institute, Utrecht, The Netherlands

Journal of the American Heart Association - submitted 


\section{6 | CHAPTER 7}

\section{ABSTRACT}

\section{Background}

The correct interpretation of the electrocardiogram (ECG) is pivotal for accurate diagnosis of many cardiac abnormalities and conventional computerized interpretation has not been able to reach physician level accuracy in detecting (acute) cardiac abnormalities yet. This study aims to develop and validate a deep neural network for comprehensive automated ECG triage in daily practice.

\section{Methods}

The 12-lead ECGs were acquired from January 2000 until August 2019 on all non-cardiology wards and outpatient clinics, the Intensive Care Unit and Emergency Department of the University Medical Center Utrecht. We developed a 37-layer convolutional residual deep neural network on a dataset of free text physician-annotated 12-lead electrocardiograms. The algorithm learned to classify these ECGs into four triage categories: normal, abnormal not acute, subacute and acute. The deep neural network was trained on a dataset with 336.835 recordings from 142.040 patients and validated on a separate consensus validation subset $(\mathrm{n}=$ 984), annotated by a panel of five cardiologists-electrophysiologists. Results were compared to the annotation of an individual physician in daily practice and the conventional computer algorithm, Marquette 12SL. Discriminative performance is presented with overall and class-specific c-statistics (equivalent to an area under the receiver operating curve) and sensitivities, specificities, positive and negative predictive values.

\section{Results}

The deep neural network demonstrated in this paper showed excellent overall discrimination with a c-statistic of 0.95 (95\% CI $0.94-0.96$ ), compared to $0.82(95 \%$ CI $0.80-0.84)$ for the individual physician in daily practice and $0.80(95 \% \mathrm{CI} 0.78-0.82)$ for the conventional Marquette $12 \mathrm{SL}$ computer algorithm. 


\section{Conclusions}

This study demonstrates that an end-to-end deep neural network can accurately be trained on unstructured free text physician annotations and used to consistently triage 12-lead electrocardiograms. When further finetuned with other clinical outcomes and externally validated in clinical practice, the demonstrated deep learning-based ECG interpretation can potentially improve time-to-treatment and decrease healthcare burden. 


\section{INTRODUCTION}

With more than 300 million ECGs obtained annually worldwide, the electrocardiogram (ECG) is a fundamental tool in the everyday practice of clinical medicine. ${ }^{1}$ The correct interpretation of the ECG is pivotal for accurate diagnosis of a wide spectrum of cardiac abnormalities and requires the expertise of an experienced cardiologist. The life-threatening nature of a suspected acute coronary syndrome and ventricular arrhythmias requires not only accurate, but also timely ECG interpretation and places a heavy logistic burden on clinical practice. Automated triage of ECGs in categories that need acute, non-acute or no attention may therefore be of great support in daily practice. Accurately prioritizing different ECGs could lead to improvements in time-to-treatment and possibly decrease healthcare costs. ${ }^{2}$ Especially in pre-hospital care and non-cardiology departments, an expert knowledge to interpret ECGs might not always be readily available..$^{3-5}$ However, a consistent and fast automated algorithm that supports the physician in comprehensive triage of the ECG remains lacking.

Computerized interpretation of the ECG (CIE) was introduced over 50 years ago and became increasingly important in aiding the physician interpretation in many clinical settings. However, current CIE algorithms have not been able to reach physician level accuracy in diagnosing cardiac abnormalities. ${ }^{5}$ Accurate interpretation of arrythmias and ST-segment abnormalities remains the most problematic and many algorithms suffer from high amounts of false positives for these disorders. ${ }^{4-9}$ Overdiagnosis and failure to correct the erroneous interpretation by overreading physician has shown to lead to unnecessary interventions and medication use. ${ }^{10,11}$

With the development of algorithms that can benefit from large-scale processing of raw data without the need for hand-crafted feature extraction, a substantial improvement of CIE is forthcoming. Several of these techniques, deep neural networks (DNN) in particular, have shown to be highly effective in similar fields as speech recognition and image classification. ${ }^{12-14}$ DNNs are computer algorithms based on the structure 
and function of the human brain. Their hidden layers of neurons can be trained to discover complex patterns in signals such as the ECG. ${ }^{15}$ In comparison to conventional CIE algorithms, DNNs have the advantage that they jointly optimize both pattern discovery and classification in an end-to-end approach that only needs the raw waveforms as input. In medicine, deep learning showed promising results when applied to arrhythmia detection in single lead ECG recordings and to early detection of atrial fibrillation in normal sinus rhythm ECGs. ${ }^{16,17}$ When combined with ultrasound or laboratory findings, deep learning algorithms were able to detect reduced ejection fraction and hyperkalemia in 12-lead ECGs. ${ }^{18,19}$ This study aims to develop and validate a DNN for comprehensive automated ECG triage that could support daily clinical practice.

\section{METHODS}

\section{Study participants}

The dataset contained 12-lead ECGs from patients between 18 and 85 years old recorded in the University Medical Center Utrecht from January 2000 to August 2019, obtained at non-cardiology departments. All extracted data were de-identified in accordance with the EU General Data Protection Regulation and written informed consent was therefore not required by the UMC Utrecht ethical committee.

\section{Training and development data acquisition and annotation}

All ECGs were recorded on a General Electric MAC 5500 (GE Healthcare, Chicago, IL, United States). We extracted raw 10 second 12-lead ECG data waveforms from the MUSE ECG system (MUSE version 8, GE Healthcare, Chicago, IL, United States). All recordings were systematically annotated by a physician as part of the regular clinical workflow. Using a text miningbased approach on the cardiologists free-text annotation, the ECGs were divided into 4 triage categories: [1] normal, [2] not acute abnormal (consultation within 24 hours), [3] subacute abnormal (consultation within 3 hours) and [4] acute abnormal (consult immediately). Text mining was performed with an in-house created dictionary, which mapped the 
free text annotation to the structured 'American Heart Association's Electrocardiography Diagnostic Statement List" using the quanteda package for R (version 3.5, R Foundation for Statistical Computing, Vienna, Austria). ${ }^{20,21} \mathrm{~A}$ panel of three electrophysiologists defined the triage category for every diagnostic statement, based on how quickly a cardiologist has to be consulted (figure 1). When multiple statements were applicable to a recording, the overall triage category was the maximum category.

\begin{tabular}{|c|c|c|c|}
\hline Normal & Abnormal, subacute & Abnormal, subacute & Abnormal, acute \\
\hline $\begin{array}{l}\text { Sinus rhythm } \\
\text { Sinus arrhythmia } \\
\text { Atrial ectopic beat } \\
\text { Ventricular ectopic } \\
\text { beat }\end{array}$ & $\begin{array}{l}\text { Sinus tachycardia } \\
-(220 \text { - age }) / \text { min } \\
\text { Sinus bradycardia } \\
<50 / \mathrm{min} \\
\text { Ectopic atrial rhythm } \\
\text { Atrial fibrillation } \\
<100 / \mathrm{min} \\
\text { Paced rhythm }\end{array}$ & $\begin{array}{l}\text { Atrial fibrillation } \\
=100 / \mathrm{min} \\
\text { Atrial flutter }\end{array}$ & $\begin{array}{l}\text { AVNRT } \\
\text { AVRT } \\
\text { Ventricular tachycardia } \\
\text { Junctional escape } \\
\text { Ventricular escape } \\
\text { Undefined rhythm }\end{array}$ \\
\hline First degree AV block & $\begin{array}{l}\text { Intraventricular con- } \\
\text { duction delay } \\
\text { Right bundle branch } \\
\text { block } \\
\text { Left bundle branch } \\
\text { block } \\
\text { Other blocks }\end{array}$ & $\begin{array}{l}\text { Second degree AV } \\
\text { block } \\
\text { QT interval } \\
>500 \mathrm{~ms}\end{array}$ & Third degree AV block \\
\hline & Previous & & $\begin{array}{l}\text { Subacute } \\
T \text { wave inversion } \\
\text { Acute } \\
\text { ST segment elevation and/ } \\
\text { or depression }\end{array}$ \\
\hline & $\begin{array}{l}\text { Nonspecific ST/T } \\
\text { abnormalities } \\
\text { Left ventricular hyper- } \\
\text { trophy }\end{array}$ & Pericarditis & \\
\hline
\end{tabular}

Figure 1. ECG diagnoses with their corresponding triage category. Triage categories as defined by the panel of electrophysiologists, with [1] normal, [2] not acute abnormal (consultation within 24 hours), [3] subacute abnormal (consultation within 3 hours) and [4] acute abnormal (consult immediately). AV atrioventricular. AVNRT atrioventricular nodal reentrant tachycardia, AVRT atrioventricular reentrant tachycardia. 


\section{Validation data acquisition}

For the validation of the DNN, a subset was annotated and triaged by the reference standard, a panel of five practicing senior electrophysiologists or cardiologists. All records were annotated by two independent annotators, who were blinded to the other annotation. In case of disagreement in the triage category, a third annotator was consulted, and the majority vote was used as the final label. The recordings with three discordant votes were discussed in a joint panel meeting and recordings of insufficient quality were excluded. To validate the performance of the algorithm with adequate precision, a minimum of 50 cases per category was needed..$^{22}$ As the smallest triage category in the training dataset has a prevalence of approximately $5 \%$, the validation dataset was a randomly sampled subset of 1000 recordings from unique patients. All recordings of these patients were excluded from the training and development datasets. The annotations that were given to the recording by a single physician in daily practice and by the conventional computer algorithm, Marquette 12SL (GE Healthcare, Chicago, IL, United States), were used for comparison. These comparison annotations were obtained by manually categorizing the free text annotation into one of the four triage categories based on figure 1 .

\section{Algorithm development}

Considering that lead III, aVR, aVL and aVF are derived from lead I and II, we used the raw 10 second 8-channel waveforms (I, II and V1-V6), sampled at $500 \mathrm{~Hz}$, as the input for our DNN. We applied an architecture similar to the Inception ResNet network, by combining blocks of convolutional layers in parallel with residual connections..$^{23,24}$ This network is built with layers of identical blocks with a pre-activation design, consisting of two 1-dimensional convolutional layers, preceded by batch normalization, rectified linear unit (ReLU) activation and dropout. ${ }^{25-27}$ Every residual block consists of three parallel branches; one with a normal convolutional layer, one with a dilated convolutional layer and a shortcut connection, where the input to the block is added to the output unadjusted. ${ }^{28}$ This enabled the network to determine features in two different time-dimensions, where the dilated convolution covered a 


\section{2 | CHAPTER 7}

complete heartbeat. The flatten layer was used as the input to a singlelayer fully connected network with a softmax layer over the 4 triage categories. An auxiliary softmax output layer was added in the middle of the network during training. Weighted focal loss was used to counteract the category imbalance in the dataset. ${ }^{29}$ Hyperparameters, such as network depth, filter sizes and downsampling, were optimized on a $5 \%$ randomly sampled development subset using a combination of manual tuning and random grid search.

The final network consisted of 16 residual blocks with two 1D convolutional layers with filter size 5 and dilation of 100 (Figure 2). Every other block downsampled the input using a strided convolution and the number of filters was doubled every fourth block. Dropout was performed with a probability of $30 \%$. This resulted in a final network with 37 layers. This network was optimized using the Adam optimizer. ${ }^{30}$ Training was terminated when the loss stopped decreasing in the development dataset. Network training was performed using the PyTorch package on an Titan Xp GPU (NVIDIA Corporation, Santa Clara, CA, United States). ${ }^{31}$ 


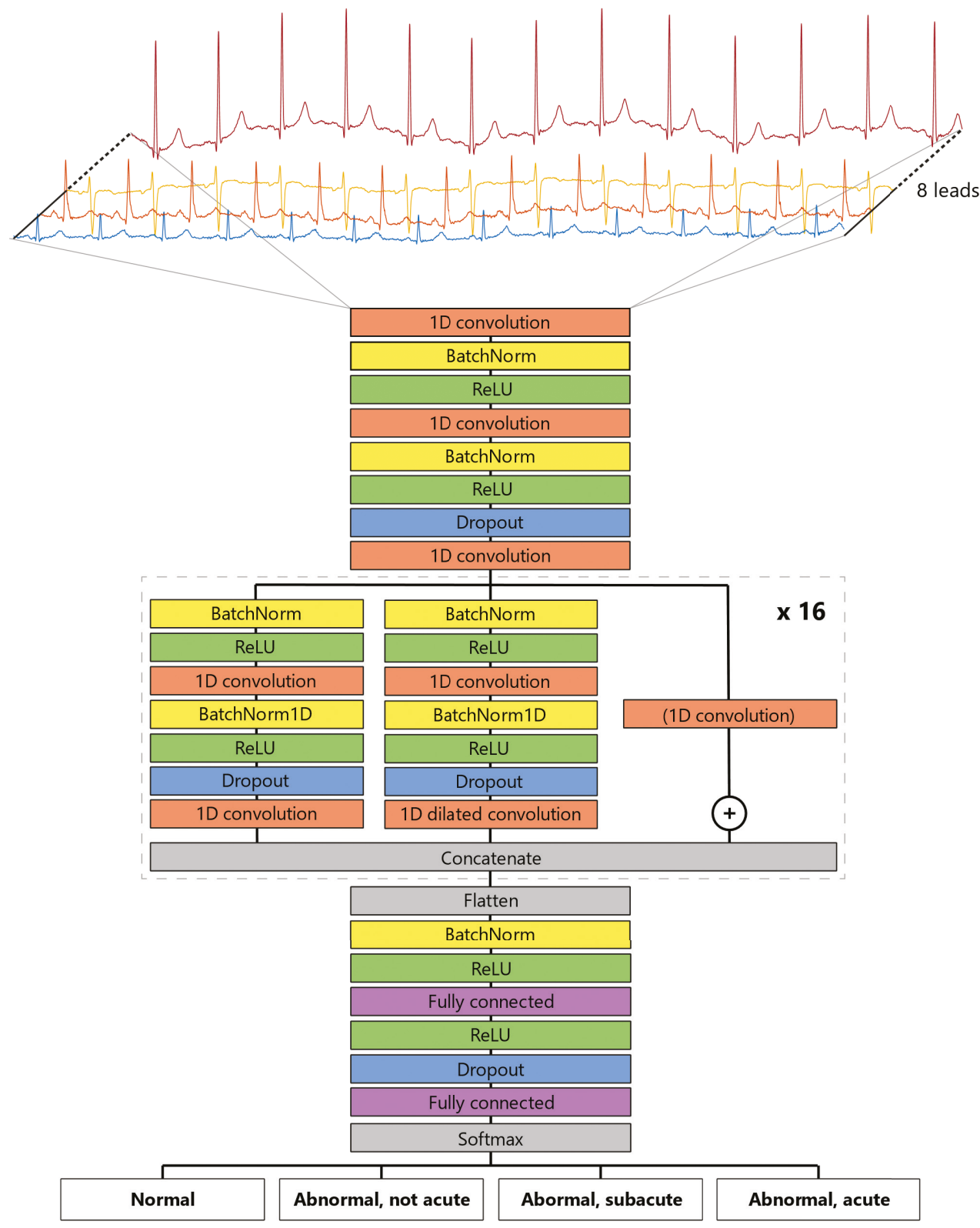

Figure 2. Design of the deep convolutional neural network. Schematic representation of the design of the 37-layer 1D residual convolutional neural network. BatchNorm: batch normalization. ReLU: rectified linear unit. 


\section{4 | CHAPTER 7}

\section{Visualization of the DNN}

To improve understanding of the decisions of the DNN, Guided Grad-CAM, a technique for visual explanations in convolutional neural networks, was adjusted for use in 1-dimensional data. ${ }^{32}$ Guided Grad-CAM is a combination between the fine-grained Guided Backpropagation and GradCAM, which produces a coarse class-discriminative heatmap based on the final convolutional layer. ${ }^{32,33}$ The heatmap is superimposed over the ECG recording and shows the regions in the ECG important to the DNN for predicting a specific triage category.

\section{Statistical analysis}

Inter-observer agreement was quantified using Krippendorff's alpha for ordinal data. ${ }^{34}$ Algorithm performance was assessed with the concordance (or c) statistic (also known as area under the receiving operating curve (AUROC)) for discriminative ability. All measures were applied in a oneversus-other approach for all triage categories. An overall measure of algorithm performance was obtained by taking the micro-averaged c-statistic.

All statistical analyses were performed using $R$ version 3.5 ( $R$ Foundation for Statistical Computing, Vienna, Austria). The TRIPOD Statement for reporting of diagnostic models was followed, where appropriate. ${ }^{35}$ All data are presented as mean \pm standard deviation or median with interquartile range (IQR). The $95 \%$ confidence intervals around the performance measures were obtained using 2000 bootstrap samples.

\section{RESULTS}

The total training dataset consisted of 336.835 recordings of 142.040 patients. The distribution of triage categories was unbalanced with the most recordings in category $2(41.5 \%)$ and the least in category $3(3.1 \%)$. In the validation dataset, there was consensus between the two experts in 736 cases (73.6\%). After consultation of a third tie-breaker expert and exclusion of recordings of insufficient quality, 984 validation cases 
were used for the analysis. There was good inter-observer agreement, with a Krippendorff's alpha of 0.72 . Conflicts in the first expert annotation round occurred the most between category 1 and 2 (162/255, 64\%), between category 2 and $3(30 / 255,12 \%)$ and between category 2 and 4 (24/255, 9.5\%). Disagreement between category 1 and 2 was mostly due to different assessments on the presence of nonspecific ST-segment or T-wave abnormalities. For category 2 and 3 and category 3 and 4, the most common difference was the interpretation of ST-segment elevation or depression. Table 1 summarizes the patient demographics and triage category distributions of the recordings in the training and validation datasets.

\begin{tabular}{|lll|}
\hline Diagnosis & $\begin{array}{l}\text { Training }(\mathbf{n}= \\
\text { 336.835) }\end{array}$ & Validation (n = 984) \\
\hline Male gender (n (\%)) & $188858(56.1)$ & $402(54.3)$ \\
Age (mean (sd)) & $60.77(15.54)$ & $60.38(15.30)$ \\
Location (n (\%)) & & \\
Emergency Department & $92532(27.5)$ & $310(31.5)$ \\
Intensive Care Unit & $20045(6.0)$ & $63(6.4)$ \\
Non-cardiac outpatient clinic & $73170(21.7)$ & $161(16.4)$ \\
Non-cardiac ward & $86630(25.7)$ & $263(26.7)$ \\
Pre-operative screening & $6300(1.9)$ & $8(0.8)$ \\
Recovery ward & $53994(16.0)$ & $163(16.6)$ \\
Other & $4164(1.2)$ & $16(1.6)$ \\
Triage category (n (\%)) & & $418(42.5)^{\mathrm{b}}$ \\
Normal & $142456(42.3)^{\mathrm{a}}$ & $410(41.7)^{\mathrm{b}}$ \\
Abnormal, not acute & $153360(45.5)^{\mathrm{a}}$ & $76(7.7)^{\mathrm{b}}$ \\
Abnormal, subacute & $24731(7.3)^{\mathrm{a}}$ & $80.1 \mathrm{~b} \mathrm{~b}$ \\
Abnormal, acute & $16288(4.8) \mathrm{a}$ & \\
\hline
\end{tabular}

Table 1. Patient demographics and distribution of triage categories in the training and validation datasets. a Distribution 


\section{6 | CHAPTER 7}

based on text mining categorization of annotations by physician in daily practice. ${ }^{b}$ Distribution based on the expert consensus panel annotation.

The discrimination, as measured by the c-statistic, of the DNN demonstrated in this paper was 0.95 (95\% CI $0.94-0.96)$, compared to $0.82(95 \%$ CI $0.80-0.84)$ for the individual physician in daily practice and 0.80 (95\% CI 0.78 - 0.82) for the Marquette 12 SL computer algorithm. C-statistics, sensitivities (Se), specificities (Sp), positive predictive values (PPV) and negative predictive values (NPV) per triage category in a onevs-all approach are shown in table 2, while the confusion matrices are appreciated in figure 3. A visualization of the regions in the ECG important for the DNN to predict a specific category is shown in figure 4.

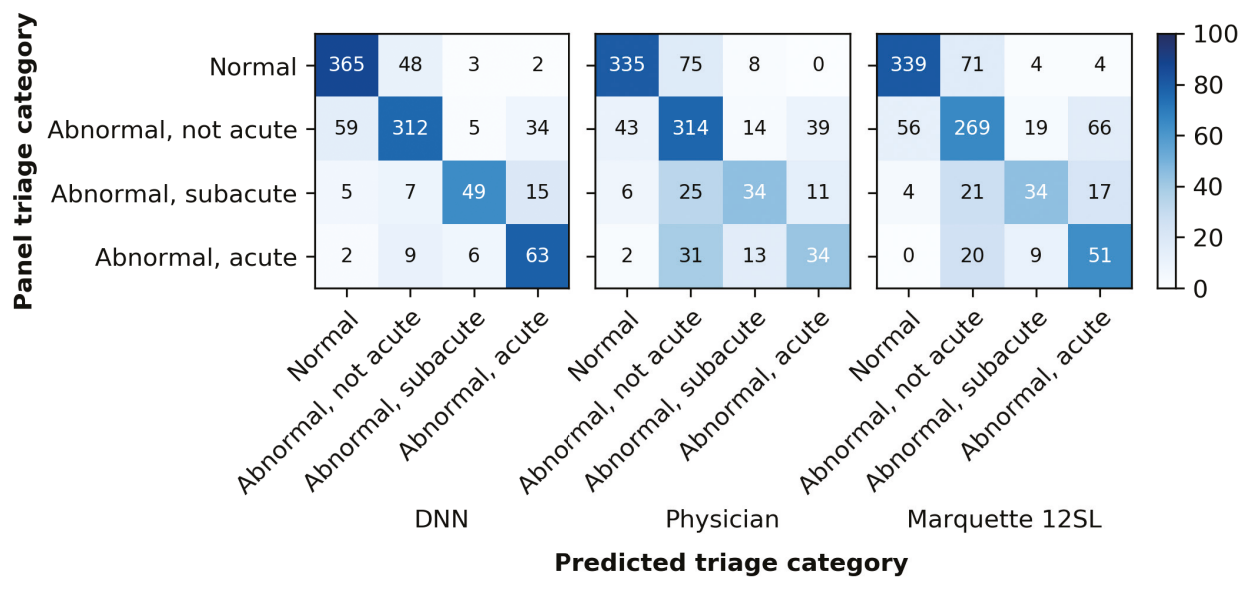

Figure 3. Confusion matrices for the deep neural network, the individual physician in daily practice and the conventional Marquette 12SL algorithm. Rows represent the categories given by the reference standard (panel) and columns the categories predicted by the deep neural network (DNN) or derived from the free text annotation by the physician in daily practice or the conventional Marquette 12SL algorithm. The colormap is normalized per row and represents the percentage in the true triage category. DNN deep neural network. 

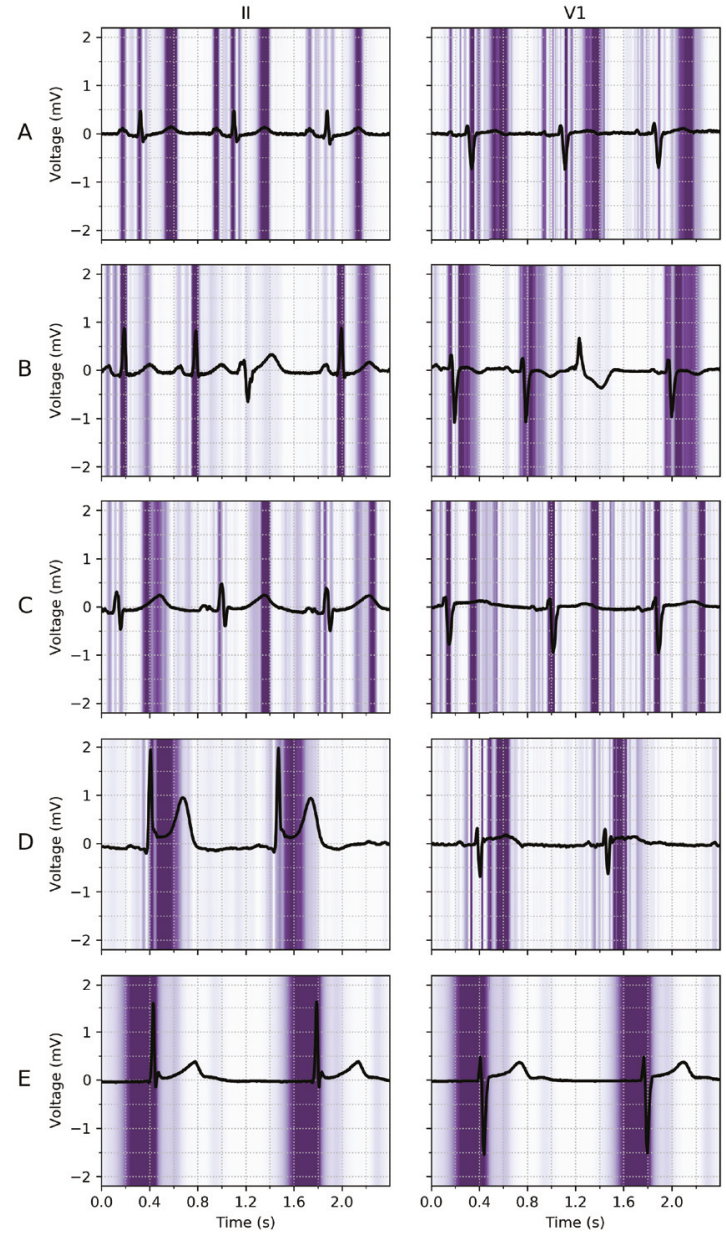

Figure 4. Examples of electrocardiogram (ECG) leads II and V1 with superimposed a Guided Grad-CAM visualization showing regions important for the deep neural network (DNN) to predict a certain triage category in purple. A Normal ECG with focus on the P-wave, QRS-complex and T-wave. B Normal ECG with a single ignored premature ventricular complex (PVC). C Subacute ECG with a long QT interval and a focus on the beginning and end of the QT-segment. D Acute ECG with an inferior ST-elevation myocardial infarction and a focus on the ST-segment and J-point. E Acute ECG with a complete atrioventricular block and a focus on the pre-QRS-segment, where the P-wave is missing. 


\begin{tabular}{|c|c|c|c|}
\hline & DNN & Physician & $\begin{array}{l}\text { Marquette } \\
\text { 12SL }\end{array}$ \\
\hline \multicolumn{4}{|l|}{ Normal } \\
\hline C-statistic (95\% CI) & $\begin{array}{l}0.95(0.94- \\
0.96)\end{array}$ & $\begin{array}{l}0.86(0.83- \\
0.88)\end{array}$ & $\begin{array}{l}0.85(0.83- \\
0.87)\end{array}$ \\
\hline Sensitivity & 0.87 & 0.80 & 0.81 \\
\hline Specificity & 0.88 & 0.91 & 0.89 \\
\hline Positive predictive value & 0.85 & 0.87 & 0.85 \\
\hline Negative predictive value & 0.90 & 0.86 & 0.87 \\
\hline \multicolumn{4}{|l|}{ Abnormal, not acute } \\
\hline C-statistic (95\% CI) & $\begin{array}{l}0.91(0.89- \\
0.93)\end{array}$ & $\begin{array}{l}0.77(0.74- \\
0.80)\end{array}$ & $\begin{array}{l}0.73(0.70- \\
0.76)\end{array}$ \\
\hline Sensitivity & 0.76 & 0.77 & 0.66 \\
\hline Specificity & 0.89 & 0.77 & 0.80 \\
\hline Positive predictive value & 0.83 & 0.71 & 0.71 \\
\hline Negative predictive value & 0.84 & 0.82 & 0.77 \\
\hline \multicolumn{4}{|l|}{ Abnormal, subacute } \\
\hline C-statistic (95\% CI) & $\begin{array}{l}0.94(0.90- \\
0.97)\end{array}$ & $\begin{array}{l}0.70(0.65- \\
0.76)\end{array}$ & $\begin{array}{l}0.71(0.65- \\
0.76)\end{array}$ \\
\hline Sensitivity & 0.64 & 0.45 & 0.45 \\
\hline Specificity & 0.98 & 0.96 & 0.96 \\
\hline Positive predictive value & 0.78 & 0.49 & 0.51 \\
\hline Negative predictive value & 0.97 & 0.95 & 0.95 \\
\hline \multicolumn{4}{|l|}{ Abnormal, acute } \\
\hline C-statistic (95\% CI) & $\begin{array}{l}0.94(0.90- \\
0.96)\end{array}$ & $\begin{array}{l}0.68(0.63- \\
0.74)\end{array}$ & $\begin{array}{l}0.77(0.72- \\
0.82)\end{array}$ \\
\hline Sensitivity & 0.79 & 0.43 & 0.64 \\
\hline Specificity & 0.94 & 0.94 & 0.90 \\
\hline Positive predictive value & 0.55 & 0.40 & 0.37 \\
\hline Negative predictive value & 0.98 & 0.95 & 0.97 \\
\hline
\end{tabular}


Table 2. Diagnostic performance measures per triage category for the deep neural network, the individual physician in daily practice and the Marquette 12SL conventional algorithm in the panel annotated validation dataset. CI: confidence interval, C-statistic: concordance statistic, equivalent to area under the receiver operating characteristic curve.

The DNN predicted a lower triage category than the true category (undertriage) in 88 (8.9\%) and a higher category (overtriage) in 107 $(11 \%)$ of the recordings in the validation dataset. Most undertriage (59/88, 67\%) occurred between categories 1 and 2 and these undertriaged recordings were categorized as 2 by the panel based on nonspecific ST-segment abnormalities (26/59, 44\%), old ischemia (12/59, $20 \%)$, left ventricular hypertrophy $(7 / 59,12 \%)$ or other reasons $(14 / 59$, $24 \%$ ). All 9 acute category 4 recordings triaged as category 2 contained ST-depression or T-wave inversion and no ST-elevation. In the category 2 recordings overtriaged as 4 , the panel did mention nonspecific ST-segment abnormalities in 20/34 (59\%) recordings and old ischemia in $8 / 34$ (24\%).

\section{DISCUSSION}

This study is among the first to apply DNNs to a large dataset of 12-lead ECGs for automatic interpretation. We demonstrated that a deep learning approach outperforms the individual physician in daily practice in detecting abnormalities for triage of 12-lead ECGs. Our DNN has an excellent c-statistic of 0.95 , with high positive and negative predictive values across all triage categories. These findings indicate that a deep learning approach may be used to support the physician in ECG triage and reduce clinical workload with an improved prioritization of ECGs for interpretation by the cardiologist.

Interpretation of the ECG requires extensive knowledge of the wide variety of electrical manifestations of heart disease and a good understanding of normal variety. This has been a challenge for both manual and computerized interpretation, and has led to a collection of definitions, 


\section{0 | CHAPTER 7}

measurements and criteria to aid clinical decision making. ${ }^{5,21}$ This challenge is also seen in this study, where the physician in daily practice has high false negative rates and the conventional computerized algorithm high false positive rates. This is in line with earlier studies, which found similar patterns. ${ }^{4-9}$ The DNN improves both the high false negative rate of physicians and the high false positive rate of conventional algorithms. Conventional CIE uses manually derived features, which only capture a fraction of the available information for any manifestation of heart disease in the obtained raw signal. This is one of the reasons that could explain the excellent performance of our algorithm and DNN in general, as their integrated feature discovery and classification incorporates the whole raw input signal. In addition, conventional CIE algorithms are tuned to produce complete interpretations of the ECG and are less focused on one of their most important uses, quick triage. By training on a large physician-annotated 12-lead ECG dataset, where the labels are mapped to predefined triage categories, we focus on a single task and are able to improve accuracy. The large size of the dataset makes that the network has seen a wide variety of ECGs and should therefore be well generalizable.

Although the DNN does not use any manually selected features of the signal, visualizations show that the network bases its decisions on same regions in the ECG as experts would. As shown in figure 4 the network correctly identifies a normal ECG, a long QT-segment, ST-elevation myocardial infarction and a complete atrioventricular block in sensible regions and correctly ignores a premature ventricular complex in a normal ECG. Furthermore, inspection of the misclassifications of the DNN shows a similar pattern to the disagreement between the experts in the panel. The correct interpretation of ST-segment and T-wave abnormalities is apparently challenging for both the cardiologist and the DNN.

Other research demonstrated the value of DNN for ECG interpretation for similar problems, where a single-lead ECG was used for arrhythmia classification and a 12-lead ECG for early detection of atrial fibrillation, contractile dysfunction and hyperkalemia. ${ }^{16-19}$ Our study shows that, in comparison to combining ECG recordings with other imaging modalities or 
laboratory findings, it is also feasible to use the less structured physician labels to successfully train a network.

Triage is the process of classifying according to the severity of the case to determine how quickly action is needed. Careful triage is needed to prioritize those cases where timely action reduces morbidity and mortality among patients. For a triage algorithm to be effective, it is important that undertriage, e.g. failure to detect patients with acute disease, and overtriage, e.g. false alarms, are minimized. Our DNN shows very high negative predictive values for the highest categories, subacute and acute (table 2). This can potentially reduce time-to-treatment for patients with acute cardiac disorders, as the algorithm is able to provide triage advice immediately after the ECG is acquired and before the ECG is assessed by a physician with sufficient expertise. In addition, the algorithm shows higher positive predictive values than the individual physician and the conventional computerized methods, which will decrease the amount of false alarms in otherwise normal ECGs. Since most hospital-acquired ECGs fall into this category, a modest improvement can already significantly decrease the workload for physicians.

This study has several limitations to address. Though a reasonable large training dataset was used, the acute categories remained relatively small. This is customary to an unselected real-world dataset but entails a chance of underprediction. We made use of the focal loss method, used in computer-vision DNN algorithms, to counteract this problem. ${ }^{26}$ In the validation dataset the triage category distribution was similar, but confidence intervals showed adequate precision in the small categories too. The representative sampling of the validation is also a strength, making it possible to derive positive and negative predictive values, which are most important to the patient. Furthermore, this network is trained on ECGs all made with the same device and filtering strategy. It remains unclear if the network will perform well when different recording devices are used, and external validation is needed to investigate this. Finally, we also note that external validation in other clinical settings is needed to show its generalizability for use outside of the hospital, such as the general practitioner, where it can be of particular use. 


\section{2 | CHAPTER 7}

Both manual and computerized ECG interpretation are hard to standardize, as can be seen by high disagreement rates between the experts (Krippendorff's alpha 0.72). This number is comparable to earlier studies on the inter-observer agreement between experts on ECG interpretation. 4,6,36 The panel-annotated validation dataset used in this study is the current best reference standard available, but in clinical practice, many other diagnostic tests are used to interpret the ECG findings. Therefore, we suspect the diagnostic accuracy of our algorithm could be further optimized with hard clinical outcome data, such as a diagnosis and localization of myocardial infarction with coronary artery angiography, cardiac enzymes and electrolyte disorders from laboratory data and even mortality. Both optimization with clinical outcome data and external validation is necessary before clinical implementation is possible. Another future perspective of the DNN is their capability to continuously improve and learn by adding new cases. Traditionally, neural networks did not provide uncertainly around their predictions, but new insights with several different Bayesian methods changed this. ${ }^{37}$ When combining uncertainty around the predictions with active learning, it becomes possible to let uncertain cases be annotated by a cardiologist and improve the algorithm, while easier cases can be classified automatically. ${ }^{38}$ In conclusion, our end-to-end DNN can triage 12-lead electrocardiograms into normal, abnormal and acute with high discrimination, outperforming the conventional computerized algorithm and the physician in daily practice. In clinical practice, this could lead to faster detection of acute disorders and decreased and better-balanced workload for clinicians. If further validated in clinical practice and other populations, the algorithm could improve time-to-treatment for acute cardiac disorders and decrease burden on daily clinical practice. 


\section{REFERENCES}

1. Holst $\mathrm{H}$, Ohlsson $M$, Peterson $C$, Edenbrandt L. A confident decision support system for interpreting electrocardiograms. Clin Physiol. 1999;19(5):410-418. doi:10.1046/j.1365-2281.1999.00195.x

2. Diercks DB, Kontos MC, Chen AY, et al. Utilization and Impact of PreHospital Electrocardiograms for Patients With Acute ST-Segment Elevation Myocardial Infarction. J Am Coll Cardiol. 2009;53(2):161-166. doi:10.1016/j.jacc.2008.09.030

3. Eslava D, Dhillon S, Berger J, Homel P, Bergmann S. Interpretation of electrocardiograms by first-year residents: the need for change. J Electrocardiol. 2009;42(6):693-697. doi:10.1016/j. jelectrocard.2009.07.020

4. Salerno SM, Alguire PC, Waxman HS. Competency in Interpretation of 12-Lead Electrocardiograms: A Summary and Appraisal of Published Evidence. Ann Intern Med. 2003;138(9):751. doi:10.7326/0003-4819138-9-200305060-00013

5. Schläpfer J, Wellens HJ. Computer-Interpreted Electrocardiograms. J Am Coll Cardiol. 2017;70(9):1183-1192. doi:10.1016/j. jacc.2017.07.723

6. Holmvang L, Hasbak P, Clemmensen P, Wagner G, Grande P. Differences between local investigator and core laboratory interpretation of the admission electrocardiogram in patients with unstable angina pectoris or non-Q-wave myocardial infarction (a thrombin inhibition in myocardial ischemia [TRIM] substudy). Am J Cardiol. 1998;82(1):54-60. doi:10.1016/S0002-9149(98)00226-4

7. Berge HM, Steine K, Andersen TE, Solberg EE, Gjesdal K. Visual or computer-based measurements: Important for interpretation of athletes' ECG. Br J Sports Med. 2014;48(9):761-767. doi:10.1136/ bjsports-2014-093412

8. Garvey JL, Zegre-Hemsey J, Gregg R, Studnek JR. Electrocardiographic diagnosis of ST segment elevation myocardial infarction: An evaluation of three automated interpretation algorithms. J Electrocardiol. 2016;49(5):728-732. doi:10.1016/j.jelectrocard.2016.04.010 
9. Shah AP, Rubin SA. Errors in the computerized electrocardiogram interpretation of cardiac rhythm. J Electrocardiol. 2007;40(5):385-390. doi:10.1016/j.jelectrocard.2007.03.008

10. Bogun F, Anh D, Kalahasty G, et al. Misdiagnosis of atrial fibrillation and its clinical consequences. Am J Med. 2004;117(9):636-642. doi:10.1016/j.amjmed.2004.06.024

11. Southern WN, Arnsten JH. The effect of erroneous computer interpretation of ECGs on resident decision making. Med Decis Mak. 2009;29(3):372-376. doi:10.1177/0272989X09333125

12. Graves A, Mohamed A, Hinton G. Speech recognition with deep recurrent neural networks. In: 2013 IEEE International Conference on Acoustics, Speech and Signal Processing. ; 2013:6645-6649. doi:10.1109/ICASSP.2013.6638947

13. Gulshan V, Peng L, Coram M, et al. Development and validation of a deep learning algorithm for detection of diabetic retinopathy in retinal fundus photographs. JAMA - J Am Med Assoc. 2016;316(22):24022410. doi:10.1001/jama.2016.17216

14. Tschandl P, Rosendahl C, Akay BN, et al. Expert-Level Diagnosis of Nonpigmented Skin Cancer by Combined Convolutional Neural Networks. JAMA Dermatology. 2018;155(1):58-65. doi:10.1001/ jamadermatol.2018.4378

15. Goodfellow I, Bengio Y, Courville A. Deep Learning. The MIT Press; 2016.

16. Hannun AY, Rajpurkar P, Haghpanahi M, et al. Cardiologistlevel arrhythmia detection and classification in ambulatory electrocardiograms using a deep neural network. Nat Med. 2019;25(January). doi:10.1038/s41591-018-0268-3

17. Attia ZI, Noseworthy PA, Lopez-Jimenez F, et al. An artificial intelligence-enabled ECG algorithm for the identification of patients with atrial fibrillation during sinus rhythm: a retrospective analysis of outcome prediction. Lancet. 2019;6736(19):1-7. doi:10.1016/S01406736(19)31721-0

18. Galloway CD, Valys A V., Shreibati JB, et al. Development and Validation of a Deep-Learning Model to Screen for Hyperkalemia From 
the Electrocardiogram. JAMA Cardiol. 2019;55905:1-9. doi:10.1001/ jamacardio.2019.0640

19. Attia ZI, Kapa S, Lopez-Jimenez F, et al. Screening for cardiac contractile dysfunction using an artificial intelligence-enabled electrocardiogram. Nat Med. 2019;25(1):70-74. doi:10.1038/s41591018-0240-2

20. Benoit $\mathrm{K}$, Watanabe $\mathrm{K}$, Wang $\mathrm{H}$, et al. quanteda: An $\mathrm{R}$ package for the quantitative analysis of textual data. J Open Source Softw. 2018;3(30):774. doi:10.21105/joss.00774

21. Mason JW, Hancock EW, Gettes LS. Recommendations for the standardization and interpretation of the electrocardiogram: Part II: Electrocardiography diagnostic statement list: A scientific statement from the American Heart Association Electrocardiography and Arrhythmias Committee, Council . Circulation. 2007;115(10):13251332. doi:10.1161/CIRCULATIONAHA.106.180201

22. Collins GS, Ogundimu EO, Altman DG. Sample size considerations for the external validation of a multivariable prognostic model: A resampling study. Stat Med. 2016;35(2):214-226. doi:10.1002/ sim.6787

23. He K, Zhang X, Ren S, Sun J. Deep Residual Learning for Image Recognition. CoRR. 2015;abs/1512.0. http://arxiv.org/abs/1512.03385.

24. Szegedy C, Ioffe $S$, Vanhoucke V, Alemi A. Inception-v4, InceptionResNet and the Impact of Residual Connections on Learning. 2016. http://arxiv.org/abs/1602.07261.

25. Ioffe S, Szegedy C. Batch Normalization: Accelerating Deep Network Training by Reducing Internal Covariate Shift. In: Bach F, Blei D, eds. Proceedings of the 32nd International Conference on Machine Learning. Vol 37. Proceedings of Machine Learning Research. Lille, France: PMLR; 2015:448-456. http://proceedings.mlr.press/v37/ ioffe15.html.

26. Srivastava N, Hinton G, Krizhevsky A, Sutskever I, Salakhutdinov R. Dropout: A Simple Way to Prevent Neural Networks from Overfitting. J Mach Learn Res. 2014;15:1929-1958. http://jmlr.org/papers/v15/ srivastava14a.html. 
27. Glorot X, Bordes A, Bengio Y. Deep Sparse Rectifier Neural Networks Xavier. Proc 14th Int Con- ference Artif Intell Stat. 2011;15.

28. Yu F, Koltun V. Multi-Scale Context Aggregation by Dilated Convolutions. 2015. http://arxiv.org/abs/1511.07122.

29. Lin TY, Goyal P, Girshick R, He K, Dollar P. Focal Loss for Dense Object Detection. Proc IEEE Int Conf Comput Vis. 2017;2017-Octob:2999-3007. doi:10.1109/ICCV.2017.324

30. Kingma DP, Ba J. Adam: A Method for Stochastic Optimization. AIP Conf Proc. 2014;1631:58-62. doi:10.1063/1.4902458

31. Paszke A, Gross S, Chintala S, et al. Automatic differentiation in PyTorch. 2017.

32. Selvaraju RR, Cogswell M, Das A, Vedantam R, Parikh D, Batra D. Grad-CAM: Visual Explanations from Deep Networks via Gradient-Based Localization. Proc IEEE Int Conf Comput Vis. 2017;2017-Octob:618-626. doi:10.1109/ICCV.2017.74

33. Springenberg JT, Dosovitskiy A, Brox T, Riedmiller M. Striving for Simplicity: The All Convolutional Net. December 2014:1-14. http:// arxiv.org/abs/1412.6806.

34. Krippendorff $\mathrm{K}$, Hayes AF. Answering the call for a standard reliability measure for coding data. Commun Methods Meas. 2007;1(1):77-89.

35. Collins GS, Reitsma JB, Altman DG, Moons KGM. Transparent Reporting of a multivariable prediction model for Individual Prognosis Or Diagnosis (TRIPOD): The TRIPOD Statement. Ann Intern Med. 2015;162(1):55. doi:10.7326/M14-0697

36. Brosnan M, La Gerche A, Kumar S, Lo W, Kalman J, Prior D. Modest agreement in ECG interpretation limits the application of ECG

screening in young athletes. Hear Rhythm. 2015;12(1):130-136. doi:10.1016/j.hrthm.2014.09.060

37. Blundell C, Cornebise J, Kavukcuoglu K, Wierstra D. Weight Uncertainty in Neural Networks. 2015;37. http://arxiv.org/ abs/1505.05424.

38. Gal Y, Islam R, Ghahramani Z. Deep Bayesian Active Learning with Image Data. 2017. http://arxiv.org/abs/1703.02910. 




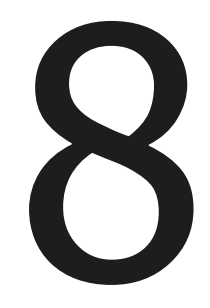

\title{
Late evolution of arrhythmogenic cardiomyopathy in patients with initial presentation as idiopathic ventricular fibrillation
}

\author{
L.J. Blom ${ }^{1}$, A.S.J.M. Te Riele ${ }^{1}$, A. Vink², R.N.W. Hauer ${ }^{1}$, R.J. Hassink ${ }^{1}$
}

1 Department of Cardiology, University Medical Center Utrecht, Utrecht, The Netherlands

2 Department of Pathology, University Medical Center Utrecht, Utrecht, The Netherlands

Published in Heart Rhythm Case Reports 2018 


\section{INTRODUCTION}

Arrhythmogenic cardiomyopathy (ACM), also known as arrhythmogenic right ventricular dysplasia/cardiomyopathy, is an inheritable heart muscle disorder in which sudden cardiac death due to ventricular fibrillation (VF) may occur unexpectedly as the first manifestation of the disease. ${ }^{1}$ This event is usually preceded by a long preclinical phase. ${ }^{2}$

Thus, prior to this event, the disease may frequently go unnoticed owing to the absence of relevant symptoms. This early presymptomatic stage has been defined as "concealed stage," which does not necessarily mean absence of disease; in the absence of symptoms, criteria for ACM diagnosis may even be already present. ${ }^{2}$ Since 2010 , ACM diagnosis is made according to revised Task Force Criteria (2010 TFC) obtained by international consensus. ${ }^{3}$ Fulfillment of these 2010 TFC (ie, presence of 2 major, 1 major plus 2 minor, or 4 minor criteria) is required for "definite" ACM diagnosis. Although fulfillment of TFC is required for definite ACM diagnosis, in the early disease stage it is conceivable that VF may occur in the presence of most but not all criteria. These cases are often regarded as idiopathic VF (IVF).

In contrast to VF due to ACM, in IVF all known cardiac, respiratory, metabolic, and toxicologic etiologies should have been excluded by the clinical evaluation available at the time of the arrhythmic event. ${ }^{4}$ However, in our experience, some survivors of initially unexplained cardiac arrest may develop "definite" ACM years after the index event, ${ }^{5}$ either by progression of the disease or by improvement of diagnostic tools. We present 2 cases of ACM patients with no or minimal disease at the time of their initial cardiac arrest episode. These episodes occurred in 1991 and 1995, thus before availability of the 2010 TFC. This means that ACM diagnosis was initially based on the less sensitive TFC published in $1994^{6}$ in 1 case, and on clinical and electrocardiogram (ECG) characteristics in the other.

\section{CASE REPORTS}




\section{Case 1}

A 24-year-old man collapsed twice in 1 year while playing in a soccer match. The first time, in 1991, he regained consciousness shortly after the start of resuscitation. Clinical evaluation was inconclusive. The second time, in 1992, he received 2 external defibrillator shocks because of VF. Resuscitation was successful and he was admitted for diagnostic evaluation. ECG showed sinus rhythm with intraventricular conduction delay, including prolonged terminal activation duration (70 $\mathrm{ms}$ ), and J-point elevation in the inferior leads (Figure 1A). History, Holter monitoring, and exercise test were unremarkable, and no ventricular extrasystoles were reported. Family history was negative for sudden cardiac death. Echocardiogram showed normal cardiac function and biventricular dimensions; however, a local abnormality under the tricuspid valve was noted. Transesophageal echocardiogram was performed, in which this abnormality was characterized as prolapse of a tricuspid valve leaflet. During electrophysiologic study, a prolonged HV interval of 60-70 ms was measured. Right ventricular (RV) stimulation induced polymorphic ventricular tachycardia (VT) starting as monomorphic VT with left bundle branch block morphology and superior axis. Other diagnostic tests, including coronary angiogram, myocardial biopsy, and ergonovine provocation test, were normal. An implantable cardioverter-defibrillator (ICD) was implanted and he was discharged. In the absence of an obvious etiology, IVF was the initial diagnosis. Cardiac magnetic resonance imaging (CMR) and signal-averaged ECG were not performed. 


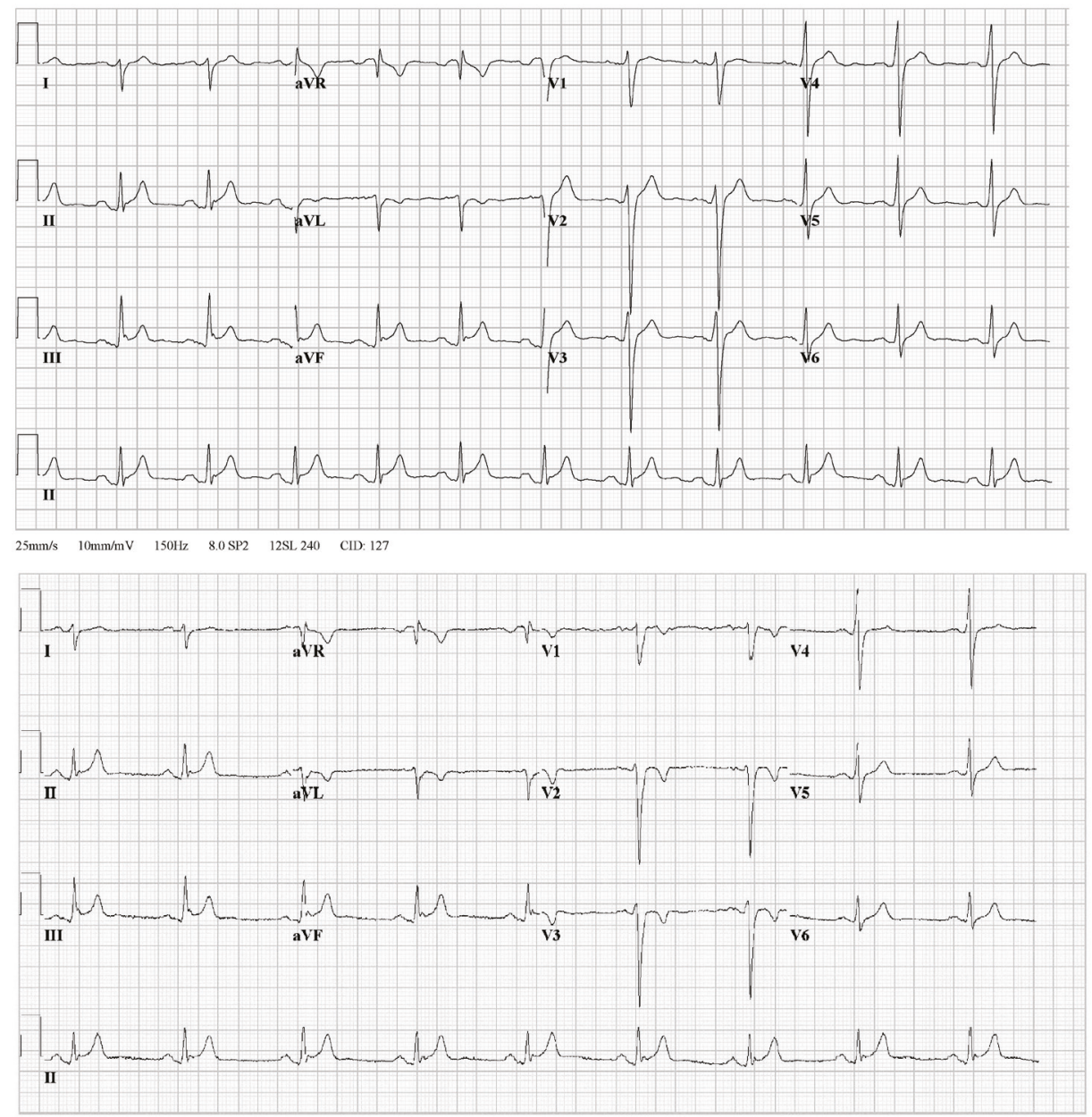

Figure 1. A A 12-lead electrocardiogram (ECG) while off antiarrhythmic drugs, showing sinus rhythm, QRS right axis deviation, QRS width 140 ms, and prolonged terminal activation duration in V2 (70 ms). Clear J-point and ST elevation in II, III, and aVF. B ECG after 10 years of follow-up, showing negative T-waves in $\mathrm{V} 1-\mathrm{V} 3$.

Ten years after the initial presentation, negative $T$ waves were recorded in V1-V3 (Figure 1B). Thus, he went on to fulfill definite ACM diagnosis criteria. On follow-up echocardiogram, there were no signs of tricuspid 
valve prolapse anymore, but careful reevaluation of imaging showed subtricuspid dyskinesia. After 16 years, RV dilatation was noted (echocardiogram 2008: dilated, hypokinetic RV; parasternal long-axis RV outflow tract [RVOT]: $32 \mathrm{~mm}$, parasternal short-axis RVOT: $35 \mathrm{~mm}$ ). Consecutive molecular genetic testing revealed an unclassified variant in the DSG2 gene and a p.Leu729del mutation in the sodium channel gene SCN5A. Familial cosegregation supported pathogenicity of this SCN5A mutation (Figure 2). During 25 years of follow-up, he remained free of ventricular tachyarrhythmias.

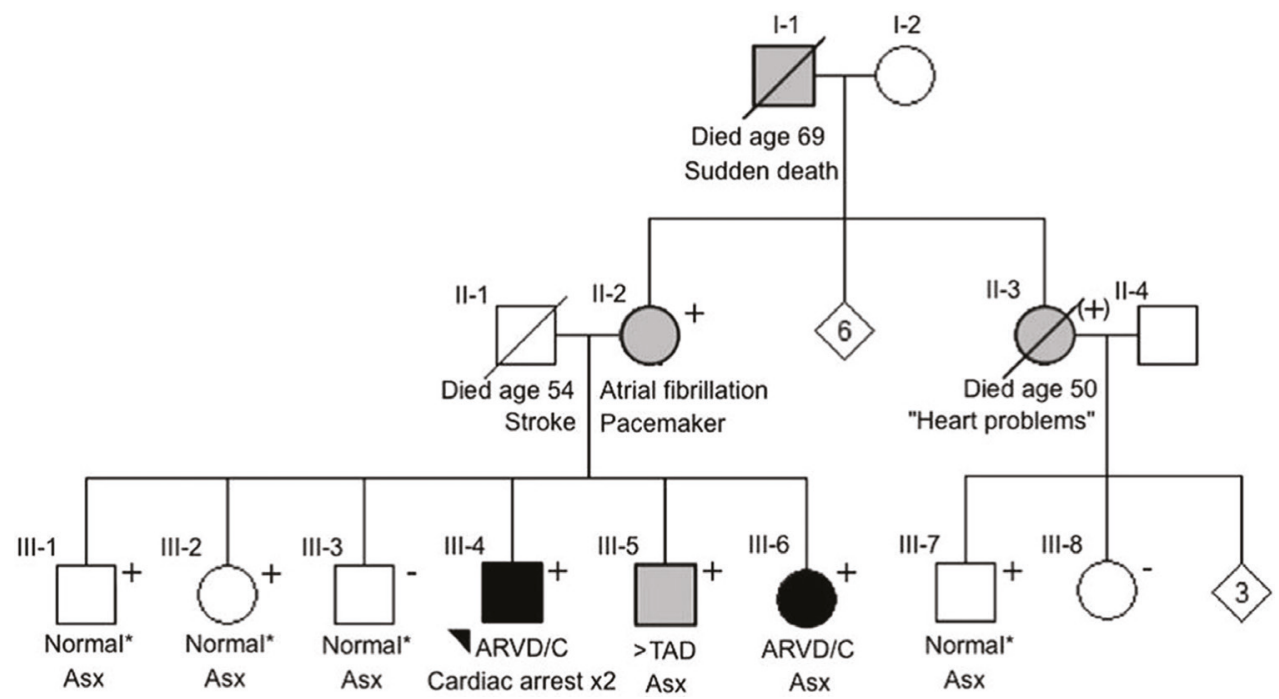

Figure 2 Pedigree of the p.Leu729del mutation carrier (case 1) reveals co-segregation of the variant with the arrhythmogenic cardiomyopathy phenotype with reduced penetrance. $A R V D / C$ arrhythmogenic right ventricular dysplasia/cardiomyopathy; $A s x$ asymptomatic; .TAD prolonged terminal activation duration. Symbols: square $=$ male; circle = female; $+=$ SCN5A mutation carrier; $(+)=$ obligate SCN5A mutation carrier; solid = clinical ARVD/C diagnosis; gray = clinical symptoms and/ or borderline ARVD/C diagnosis; empty = negative phenotype for ARVD/C. *No abnormalities on comprehensive cardiac evaluation including 12-lead electrocardiogram, Holter monitoring, and cardiac imaging. (From Te Riele and colleagues. ${ }^{14}$ ) 


\section{4 | Chapter 8}

In this case, fulfillment of 2010 TFC in follow-up was owing to disease progression (eg, progression of precordial T-wave inversions), misinterpretation of clinical evaluation (eg, wrong interpretation of subtricuspid dyskinesia as tricuspid valve prolapse), and incomplete clinical evaluation (no CMR was performed).

\section{Case 2}

In 1995, a 25-year-old man collapsed during light chores while at work in a café. He was resuscitated and admitted to the intensive care unit. His history was unremarkable and there was no sudden death in his family. Electrocardiography showed sinus rhythm with negative $T$ waves in leads V4-V6 and low voltage in extremity leads (Figure 3A). Echocardiogram, coronary angiogram, and myocardial biopsy were normal. The left ventricular ejection fraction was $62 \%$ by echocardiography. During electrophysiologic study, RVOT stimulation showed inducibility of a monomorphic VT with left bundle branch block morphology and vertical axis. An ICD was implanted and he was discharged. Thus, at initial evaluation, he fulfilled 2 minor TFC for ACM diagnosis, indicating IVF as initial diagnosis.

During follow-up, he experienced appropriate ICD shocks because of VT episodes. Subsequent Holter monitoring showed 870 premature ventricular complexes in 24 hours. Fourteen years after the event, he showed signs of heart failure with dilated ventricles (Figure 3B, echocardiogram 2009: RVOT/RV lateral wall akinesia, inferior RV dyskinesia, parasternal long-axis RVOT: $56 \mathrm{~mm}$, parasternal short-axis RVOT: $59 \mathrm{~mm}$, and left ventricular ejection fraction 25\%). Genetic testing revealed an unclassified variant in the plakophilin- 2 gene and a pathogenic c.40-42delAGA mutation in the phospholamban (PLN) gene. CMR and signal-averaged ECG were not performed. 
Late evolution of ACM in patients with initial presentation as IVF | 145
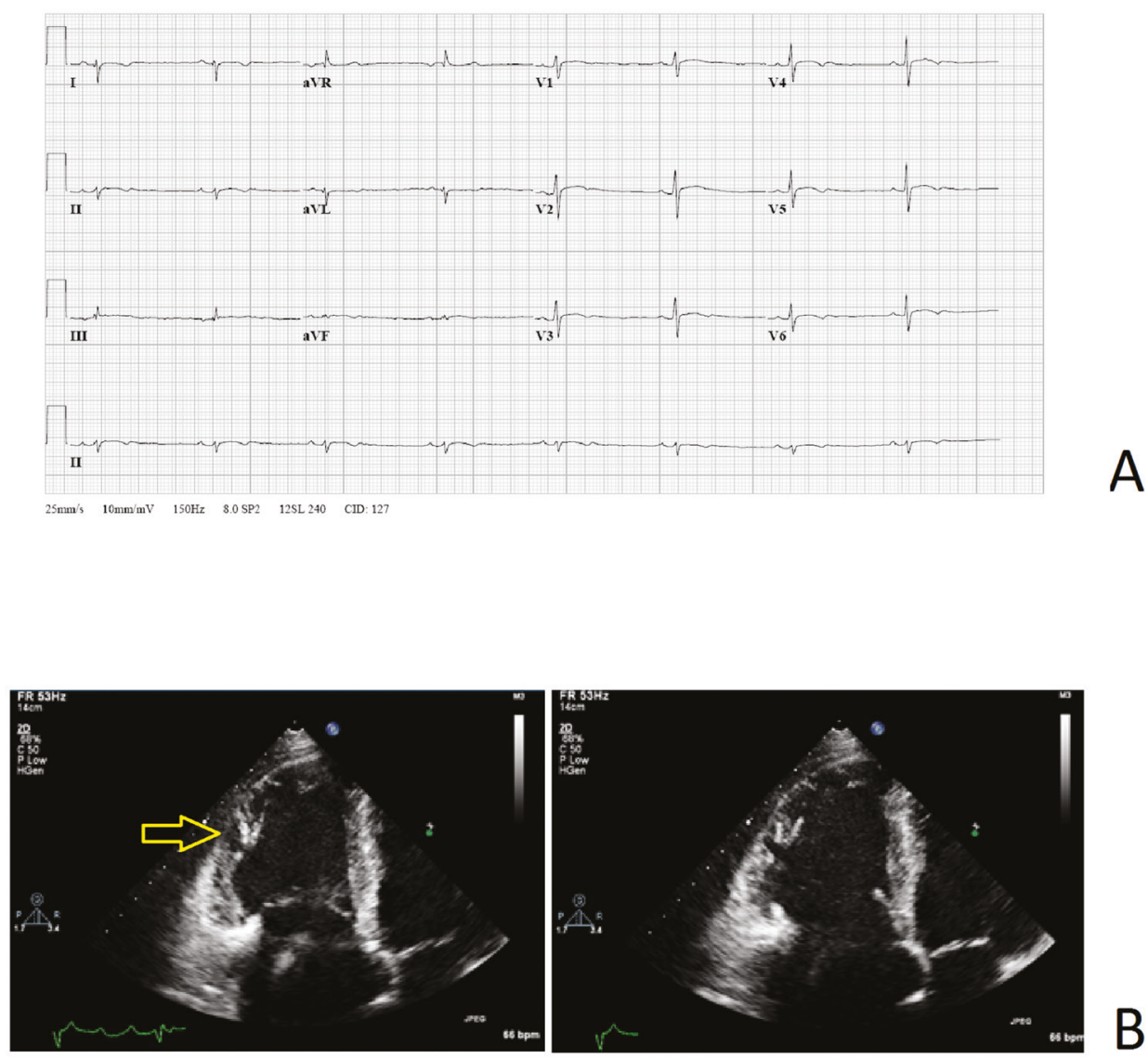

Figure 3 A A 12-lead low-voltage electrocardiogram while off antiarrhythmic drugs showing normal sinus rhythm and QRS right axis deviation. Negative T waves in left precordial recordings and I, II, and aVF. B Transthoracic echocardiogram during follow-up showing a dilated right ventricle with subtricuspid aneurysm (arrow).

Fulfillment of ACM diagnosis criteria during follow-up was owing to disease progression (eg, clinical signs of heart failure and increase in echocardiographic measurements during follow-up) and misinterpretation of clinical evaluation (eg, under-recognition of biventricular/left-dominant disease in ACM at time of first symptoms). 


\section{DISCUSSION}

\section{Absence of diagnosis does not mean absence of disease}

We present 2 cases with an initial presentation of nonfatal cardiac arrest due to underlying VF. Since only nonspecific minor abnormalities were identified at the time of presentation, these cases were diagnosed as IVF. Many years after the initial presentation, TFC fulfillment led to a definite diagnosis of ACM (Table 1).

The presented cases suggest that IVF or "concealed" ACM does not necessarily mean absence of disease, and periodic reevaluation of suspected ACM cases may lead to a definite diagnosis. Diagnosing ACM in former IVF patients is important, as it provides additional management options such as pharmacologic therapy and lifestyle interventions. ${ }^{2}$ More importantly, in family members of patients with a genetic disorder, a correct diagnosis may pave the way for cascade screening as a first step toward arrhythmic risk stratification.

\section{Reevaluation of diagnosis}

IVF is a diagnosis by exclusion in which initial evaluation does not identify an underlying cause. Diagnosing a specific disease, such as ACM, during follow-up may be a consequence of (1) improvement in diagnostic tools, (2) disease progression, or (3) misinterpretation of initially available data. These are common challenges in clinical practice, and a combination of these is illustrated in this report; our cases may be part of a larger patient population that is not diagnosed at the time of clinical presentation. 


\begin{tabular}{|c|c|c|c|c|}
\hline & \multicolumn{2}{|l|}{ Case 1} & \multicolumn{2}{|l|}{ Case 2} \\
\hline & $\begin{array}{l}\text { Initial evalu- } \\
\text { ation }\end{array}$ & $\begin{array}{l}\text { Follow-up } \\
\text { period }\end{array}$ & $\begin{array}{l}\text { Initial evalua- } \\
\text { tion }\end{array}$ & $\begin{array}{l}\text { Follow-up } \\
\text { period }\end{array}$ \\
\hline \multicolumn{5}{|l|}{1994 TFC } \\
\hline Major criteria & $\begin{array}{l}1 \\
\text { QRS dura- } \\
\text { tion > } 110 \\
\text { ms }\end{array}$ & 1 & - & $\begin{array}{l}1 \\
\text { Severe dilata- } \\
\text { tion of RV }\end{array}$ \\
\hline Minor criteria & $\begin{array}{l}1 \\
\text { VT with } \\
\text { LBBB mor- } \\
\text { phology }\end{array}$ & $\begin{array}{l}2 \\
\text { Negative } \\
T \text { waves in } \\
V_{1}-V_{3}\end{array}$ & $\begin{array}{l}1 \\
\text { VT with LBBB } \\
\text { morphology }\end{array}$ & 1 \\
\hline Diagnosis & No diagnosis & ACM & No diagnosis & $\mathrm{ACM}$ \\
\hline \multicolumn{5}{|l|}{2010 TFC } \\
\hline Major criteria & $\begin{array}{l}1 \\
\text { VT with } \\
\text { LBBB mor- } \\
\text { phology and } \\
\text { superior axis }\end{array}$ & $\begin{array}{l}2 \\
\text { Negative } \\
T \text { waves in } \\
V_{1}-V_{3}\end{array}$ & - & $\begin{array}{l}1 \\
\text { Regional } \\
\text { akinesia and } \\
\text { PLAX RVOT > } \\
32 \mathrm{~mm}\end{array}$ \\
\hline Minor criteria & $\begin{array}{l}1 \\
\text { Prolonged } \\
\text { TAD }\end{array}$ & 1 & $\begin{array}{l}2 \\
\text { Negative } \mathrm{T} \\
\text { waves in } \mathrm{V}_{4}-\mathrm{V}_{6 ;} \text {; } \\
\text { VT with LBBB } \\
\text { morphology }\end{array}$ & 2 \\
\hline ACM diagnosis & Borderline & Definite & Possible & Definite \\
\hline
\end{tabular}

Table 1 Overview of Task Force Criteria fulfilled per case.

ACM arrhythmogenic cardiomyopathy; $\angle B B B$ left bundle branch block; $P L A X$ parasternal long axis; $R V$ right ventricle; $R V O T$ right ventricular outflow tract; TAD terminal activation duration; TFC Task Force Criteria; $V T$ ventricular tachycardia.

\section{Improvement in diagnostic tools}

The ability of diagnostic tests to detect electrical and structural 
abnormalities in the initial evaluation of patients suspected of ACM varies considerably. Qualitative assessment of RV angiogram relies on clinical experience, diagnostic sensitivity of myocardial biopsy is limited by the segmental nature of disease, ${ }^{3}$ and echocardiography's performance is significantly less compared to CMR in detecting abnormalities fulfilling the 2010 TFC. ${ }^{7}$

In both these presented cases, CMR was not performed, which may have impacted our ability to fully appreciate the structural phenotype in these individuals. The improved availability in recent years of CMR has made the detection of ACM with a subtler phenotype possible. Based on this information and our clinical experience, we strongly suggest a comprehensive CMR be performed in the work-up of a patient with presumed IVF and used for evaluation of ACM diagnosis in suspected cases.

\section{Progressive disease}

ACM is a progressive disease, ${ }^{8}$ and its disease course in desmosomal mutation carriers is well studied. ${ }^{9} \mathrm{~A}$ long-lasting asymptomatic phase precedes the clinical phase in most ACM patients, in which electrical abnormalities tend to precede detectable structural changes. Moreover, presence of both an electrical and structural substrate identifies patients at high risk for arrhythmic events. ${ }^{10}$

Accordingly, diagnosis of ACM according to the 2010 TFC is based on electrical, structural, genetic, and histologic abnormalities ${ }^{3}$ and requires extensive diagnostic testing and periodic reevaluation in suspected cases. In case 1 , right precordial T-wave inversion and echocardiographic RV dilatation after 10 years of follow-up are indicators of disease progression, facilitating ACM diagnosis.

\section{Misinterpretation of initially available data}

Our knowledge of the underlying disease process and associated pathogenic mutations has facilitated diagnosis of more subtle and heterogeneous clinical presentations of ACM. Retrospectively, subtle abnormalities could be misinterpreted during initial evaluation, as is 
illustrated by both our cases.

In both patients, pathogenic mutations were found in nondesmosomal genes. The involvement of nondesmosomal genes in ACM has become clearer in recent years. For example, overlap of desmosomal and sodium channel disease (eg, ACM and Brugada syndrome) is an emerging area of interest (as described in case 1$)^{11}$; the phospholamban gene mutation found in case 2 is associated with both an arrhythmogenic and dilated cardiomyopathy phenotype. ${ }^{12}$

In case 2, increased awareness for biventricular/left-dominant forms in the new 2010 TFC and the availability of molecular genetic testing facilitated the diagnosis during follow-up. In case 1 , already at baseline, abnormal wall motion in the subtricuspid region was noted, which was falsely interpreted as tricuspid valve prolapse. Since the subtricuspid region is a hotspot region for early structural changes in $\mathrm{ACM},{ }^{13}$ overt ACM was likely already present, albeit not recognized.

\section{CONCLUSION}

VF may be the first clinical manifestation of ACM, as shown in this report. Appropriate diagnosis at later disease stages may be due to (1) improvement in diagnostic tools, (2) disease progression, or (3) misinterpretation of initially available data. In a VF survivor, a specific ACM diagnosis may be clinically less relevant for the index patient. However, in family members, a correct diagnosis may pave the way for cascade screening as a first step toward arrhythmic risk stratification. To detect affected family members before occurrence of ventricular arrhythmias and sudden death, periodic reevaluation of suspected ACM cases during follow-up is important. 


\section{REFERENCES}

1. Bhonsale A, Groeneweg JA, James CA, et al. Impact of genotype on clinical course in arrhythmogenic right ventricular dysplasia/ cardiomyopathy associated mutation carriers. Eur Heart ] 2015;36:847855.

2. Corrado D, Basso C, Thiene G. Arrhythmogenic right ventricular cardiomyopathy. Heart 2000;83:588-595.

3. Marcus FI, McKenna WJ, Sherrill D, et al. Diagnosis of arrhythmogenic right ventricular cardiomyopathy/dysplasia. Circulation 2010;121:15331541.

4. Visser M, van der Heijden JF, Doevendans PA, Loh P, Wilde AA, Hassink RJ. Idiopathic ventricular fibrillation. Circ Arrhythm Electrophysiol 2016; 9:e003817.

5. Visser M, van der Heijden JF, van der Smagt JJ, Doevendans PA, Wilde AA, Loh P, Hassink RJ. Long-term outcome of patients initially diagnosed with idiopathic ventricular fibrillation. Circ Arrhythm Electrophysiol 2016;9:e004258.

6. McKenna WJ, Thiene G, Nava A, Fontaliran F, Blomstrom-Lundqvist C, Fontaine G, Camerini F. Diagnosis of arrhythmogenic right ventricular dysplasia/cardiomyopathy. Heart 1994;71:215-218.

7. Borgquist $R$, Haugaa $K H$, Gilljam T, Bundgaard $H$, Hansen J, Eschen O, Jensen HK, Holst AG, Edvardsen T, Svendsen JH, Platonov PG. The diagnostic performance of imaging methods in ARVC using the 2010 task force criteria. Eur Heart J Cardiovasc Imaging 2014;15:1219-1225.

8. Mast TP, James CA, Calkins $\mathrm{H}$, et al. Evaluation of structural progression in arrhythmogenic right ventricular dysplasia/cardiomyopathy. JAMA Cardiol 2017;2:293.

9. Basso C, Corrado D, Marcus FI, Nava A, Thiene G. Arrhythmogenic right ventricular cardiomyopathy. Lancet 2009;373:1289-1300.

10. Te Riele ASJM, Bhonsale A, James CA, et al. Incremental value of cardiac magnetic resonance imaging in arrhythmic risk stratification of arrhythmogenic right ventricular dysplasia/cardiomyopathy-associated desmosomal mutation carriers. J Am Coll Cardiol 2013;62:1761-1769. 
11. Corrado D, Zorzi A, Cerrone M, Rigato I, Mongillo M, Bauce B, Delmar M. Relationship between arrhythmogenic right ventricular cardiomyopathy and brugada syndrome. Circ Arrhythm Electrophysiol 2016;9:1-10.

12. Van Der Zwaag PA, Van Rijsingen IAW, Asimaki A, et al. Phospholamban R14del mutation in patients diagnosed with dilated cardiomyopathy or arrhythmogenic right ventricular cardiomyopathy. Eur J Heart Fail 2012; 14:1199-1207.

13. te Riele ASJM, James CA, Philips B, et al. Mutation-positive arrhythmogenic right ventricular dysplasia/cardiomyopathy. J Cardiovasc Electrophysiol 2013; 24:1311-1320.

14. Te Riele ASJM, Agullo-Pascual E, James CA, et al. Multilevel analyses of SCN5A mutations in arrhythmogenic right ventricular dysplasia/ cardiomyopathy suggest non-canonical mechanisms for disease pathogenesis. Cardiovasc Res 2017;113:102-111. 

Chapter

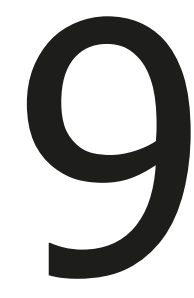

Discussion 


\section{PROGRESS IN DIAGNOSIS AND MANAGEMENT OF IDIOPATHIC VENTRICULAR FIBRILLATION}

In the preceding decade, the number of idiopathic ventricular fibrillation (IVF) patients who received a diagnosis during follow-up has increased and this number is expected to increase further in the future. ${ }^{1}$ Progress was made in diagnostic evaluation after sudden cardiac arrest, imaging techniques and understanding the underlying pathology. 2,3 Despite these improvements, a group of patients remains without diagnosis and management of this group proved to be difficult due to an insufficient understanding of the underlying mechanism of ventricular arrhythmias.

This thesis has two principal aims: 1) to expand and improve current diagnostics for idiopathic ventricular fibrillation and 2) to evaluate current management of idiopathic ventricular fibrillation patients and family members. To achieve these aims, we reviewed the current diagnostics and management of IVF patients and SCD victims (Chapter $\mathbf{2}$ and $\mathbf{3}$ ). We studied the incidence of ICD therapy and complications (Chapter 4) and the yield of family cascade screening (Chapter 5) in IVF patients and their family members. We explored the value of novel repolarization parameters in ECG imaging (Chapter 6) and deep learning algorithms in ECG triage (Chapter 7). Finally, we demonstrated the value of uncovering a diagnosis and its impact on management in IVF (Chapter 8). The findings of this thesis are put into perspective in the following sections.

\section{DIAGNOSTIC TESTING AFTER SUDDEN CARDIAC ARREST}

Sudden cardiac arrest (SCA) is a devastating event and despite great efforts survival rates remain low. The majority of events happen in the older population ${ }^{4}$ and its major cause is coronary artery disease, accounting for $>80 \%$ of SCA. ${ }^{5}$ In the remaining population a variety of extracardial causes, structural and electrical heart disease has to be excluded 6 and this requires extensive diagnostic testing. ${ }^{2}$ When no apparent cause is found, this is referred to as idiopathic ventricular 
fibrillation in survivors or sudden arrhythmic death syndrome in the deceased. ${ }^{6,7}$

In Chapter 2, $\mathbf{3}$ and $\mathbf{8}$ we discuss the need for standardized and extensive diagnostic testing in both groups of sudden cardiac arrest victims. Lack of a comprehensive work-up is a common issue in realworld IVF cohorts ${ }^{1}$ and while there have been efforts to standardize the required diagnostic tests 2,8 this has proven to be difficult, resulting in a heterogeneous study population. ${ }^{1,9-15}$ This is even more prominent in sudden cardiac death, were post-mortem diagnostic possibilities are limited ${ }^{16}$ and several definitions are used on the basis of the diagnostics performed (e.g. sudden cardiac death (SCD), sudden unexplained death (SUD) and sudden arrhythmic death syndrome (SADS)). ${ }^{7,17,18}$

In Chapter $\mathbf{2}$ we discuss the challenges in diagnosis and management of idiopathic ventricular fibrillation in practice. We advocate the need for extensive diagnostic testing since the lack of an underlying diagnosis offers few curative options due to unknown underlying pathology. We support the progress towards arrhythmic risk stratification using newly developed diagnostic tests. Extensive diagnostic testing is required for the diagnosis of IVF, $^{6}$ though in real-world settings these requirements are not always met. Waldmann et $\mathrm{al}^{1}$ describe the investigations performed in a large cohort of SCA survivors and show that many required diagnostics have not been performed in IVF patients. In retrospective cohort studies of IVF patients ${ }^{8,9,19}$ this is an important limitation. Absence of an underlying diagnosis means patients are denied therapeutic treatment options (for example betablocker therapy in long QT syndrome/ catecholamiergic polymorphic ventricular tachycardia ${ }^{6,20,21}$, avoiding certain drugs and lifestyle advice in Brugada syndrome and arrhythmogenic cardiomyopathy ${ }^{22,23}$ ) for prevention of arrhythmia recurrence or heart failure and their family members at risk do not receive recommended genetic/phenotypic screening for inherited disease. ${ }^{6,24}$ The lack of a known underlying substrate for arrhythmias also prevents comprehensive risk stratification in these patients.

Diagnostic evaluation should not stop after the initial evaluation. Followup studies of IVF cohorts show a high percentage of diagnoses found 
during follow-up..$^{9,10}$ These include many cardiomyopathies and primary arrhythmia syndromes where family members may be at risk. VF may be the first clinical manifestation of inherited disease, as shown in Chapter 8. Appropriate diagnosis at later disease stages may be due to improvement in diagnostic tools, disease progression, or misinterpretation of initially available data. It may not always benefit the VF survivor, as not all underlying diagnosis may require different management of the index patient. However, in family members, a correct diagnosis may pave the way for cascade screening as a first step toward arrhythmic risk stratification. To detect affected family members before occurrence of ventricular arrhythmias and sudden death, periodic reevaluation of idiopathic ventricular fibrillation patients during follow-up is important. Chapter $\mathbf{3}$ shows a similar relevance of diagnostic tests in young sudden cardiac death victims. A dedicated and focused post-mortem investigation is essential for detecting potential inherited cardiac diseases in young sudden death victims, which facilitates appropriate management of the victim's family members. Uncovering the cause of death in young sudden unexplained death victims requires a thorough autopsy and targeted genetic testing, which together have a high yield of uncovering inherited disease in these families..$^{25,26}$ This facilitates cascade screening and phenotypic evaluation of family members, thereby facilitating a timely diagnosis of inherited disease and preventing SCD and disease progression in those cases.

\section{MANAGEMENT OF IDIOPATHIC VENTRICULAR FIBRILLATION PATIENTS AND THEIR FAMILY MEMBERS}

Management options for idiopathic ventricular fibrillation patients are sparse and research on this topic is limited. ${ }^{12,19,27,28}$ Implantable cardioverter-defibrillator (ICD) implantation is currently the treatment of choice. ${ }^{6,29}$ Drug and ablation therapy have shown benefit in subgroups of IVF patients. ${ }^{12,30}$ Even though ICD implantation is effective in treating ventricular arrhythmias in general, it is also associated with risk of inappropriate therapy and complications. In Chapter $\mathbf{4}$ we found that 
approximately one-third of patients received appropriate implantable cardioverter-defibrillator therapy during long-term follow-up, confirming the role of the ICD as an effective treatment in IVF by treating recurrences of potentially lethal ventricular arrhythmias. We also found $17.5 \%$ of patients received inappropriate ICD therapy and over 14\% experienced device-related complications. As these numbers show, no treatment comes without unwanted side-effects and improved patient selection for ICD implantation could reduce the number of patients exposed to these risks. We found three independent predictors of appropriate shock occurrence: Symptoms before cardiac arrest [hazard ratio (HR): 2.51, 95\% confidence interval (CI): 1.48-4.24], signs of conduction disease [HR: $2.27,95 \% \mathrm{CI}: 1.15-4.47$, and carrier of the DPP6 risk haplotype [HR: 3.24, 95\% CI: 1.70-6.17]. This may imply that these patients require additional management to prevent recurrent events and, though we can only speculate on the underlying mechanisms of increased risk in these groups, they provide an important starting point for arrhythmic risk stratification and personalized therapy in IVF.

Management of family members is another important aspect in IVF patient management due to the overlap with heritable cardiac disease and primary arrhythmia syndromes that have a clear genetic base. ${ }^{31,32}$ Chapter $\mathbf{5}$ describes the role of familial cascade screening in idiopathic ventricular fibrillation. Familial cascade screening is well established in patients with heritable cardiac disease and in cases of Sudden Arrhythmic Death Syndrome (SADS). ${ }^{6,25}$ The assessment of family members of survivors of idiopathic ventricular fibrillation is not well established. Our study shows that the yield of familial cascade screening in idiopathic VF is low: an inherited arrhythmia syndrome was diagnosed in 5 (3\%) individuals from 4 (4\%) families. Two relatives were found to carry the DPP6 risk haplotype identified in the familial proband. In three separate families the parent of the proband was diagnosed with Brugada syndrome following a positive ajmaline provocation test. These findings are in contrast to the sparse evidence currently available on family screening in IVF where consistently higher yields are reported. ${ }^{33-35}$ Most of these differences can be attributed to lenient inclusion criteria for probands, who 
did not all undergo extensive diagnostic evaluation. The higher yield in family members can be explained by the high prevalence of undiagnosed disease in the proband. Study outcomes were also defined differently: Early repolarization syndrome was either excluded or defined as a heritable cardiac disease in different studies. ${ }^{33}$ The low yield of inherited disease we found in well-characterized IVF families has important consequences for management, as the current recommendation to perform phenotypic screening with ECG, echo and exercise test in all first degree relatives of IVF patients might not be appropriate.

\section{NOVEL METHODS FOR UNCOVERING ARRHYTHMOGENIC SUBSTRATE}

Conventional diagnostic tests have not been able to uncover arrhythmogenic substrate in idiopathic ventricular fibrillation. This provides an opportunity for new techniques to identify the underlying pathology. A promising non-invasive technique to map arrhythmic substrate is electrocardiographic imaging, ${ }^{36}$ which has been studied in several other arrhythmogenic diseases. ${ }^{37-40}$ In Chapter 6 we studied the novel use of repolarization parameters in electrocardiographic imaging and we hypothesized that repolarization characteristics, such as local electrogram morphology and variation in repolarization timing and duration could be of significance. These characteristics have already been proven useful in arrhythmic risk stratification using the 12-lead ECG in other arrhythmogenic diseases. ${ }^{41-43}$ We found that measuring these novel repolarization parameters in reconstructed electrograms acquired with ECGI is feasible, can be done in a fully automated manner and may provide additional information on underlying arrhythmogenic substrate for risk stratification. Most prominent findings were steep repolarization time gradients in regions with flat T-waves on the reconstructed electrograms. These regions were also characterized by low T-wave area and large differences in Tpeak-Tend interval. The significance of these findings should be further explored in clinical studies.

Detecting (undiscovered) features in data to predict outcome is a task 
that deep convolutional neural networks (DNNs) perform very well. ${ }^{44}$ In Chapter 7 we have studied the performance of DNNs in classifying on 12-lead ECG data in four triage categories. The deep neural network demonstrated in this paper showed excellent overall discrimination with a c-statistic of 0.95 (95\% CI $0.94-0.96)$, outperforming the individual physician in daily practice and the conventional computer algorithm. We demonstrated that an end-to-end deep neural network can accurately be trained on unstructured free text physician annotations and used to consistently triage 12-lead electrocardiograms. In the context of idiopathic ventricular fibrillation, this is an important finding, as the neural network is capable of extracting both known and unknown features from the electrocardiogram to improve its predictions. ${ }^{45}$ The hypothesis that there are unknown features present in the electrical heart activity data recorded by the electrocardiogram is plausible. The small cohort size and heterogeneous nature of IVF may limit the application of machine learning algorithms in this group. Though there are developments in the deep learning field to adapt networks to overcome these limitations using pre-trained models ${ }^{46,47}$ and they may help our diagnostic accuracy and understanding (through visualization techniques such as gradCAM ${ }^{48}$ ) of the underlying arrhythmogenic substrate in IVF.

\section{FUTURE DIRECTIONS}

Diagnostic testing is the crux of management and diagnosis of idiopathic ventricular fibrillation. The progress in both imaging modalities and genetic testing we have seen in the past decades has not come to a halt yet, but rather continues to improve. The rise of data-driven healthcare strategies has far from reached its full potential and could personalize risk stratification and treatment using all available data sources.

Currently, an arrhythmogenic substrate for IVF is lacking. Until now, imaging and mapping techniques have not been able to identify a substrate in this group. However, the advancements in resolution and accuracy of invasive and non-invasive imaging techniques facilitates more detailed characterization of the electrical properties of the 
myocardium. ${ }^{38,49-52}$ Future clinical studies are needed to prove the existence of arrhythmogenic substrate in these patients.

In IVF patients who have a family history of SCA / premature death there is likely an inheritable component that attributed to the arrhythmic risk. Though some monogenetic causes have been found in IVF, 53,54 polygenetic causes are likely present in the affected families where no single mutation has been found. ${ }^{55}$ Innovations in the field of genomics, genome-wide association studies and development of polygenetic risk scores could improve the management of these families by screening for affected family members and arrhythmic risk stratification. Genome-wide association studies could facilitate the development of polygenetic risk scores, ${ }^{56}$ though considering the rarity and heterogeneity of IVF cohorts this may prove to be a challenging task.

Accurately predicting the risk of sudden cardiac arrest in the healthy population is currently not possible. Due to the complex and multifactorial aetiology of this condition, there are simply too many underlying variables to consider. This is an area where the recent advancements in the fields of big data analytics can help to overcome this clinical problem. ${ }^{57}$ The vast increase in available data from all sources, including healthy individuals, can be used as input for prediction algorithms that are able to handle numerous variables and interactions. They might be able to provide insight in complex cases and suggest causes that initially wouldn't have been considered. Thereby shaping the future direction of research in this field and hopefully contribute to discovery of underlying substrate in thus far idiopathic ventricular fibrillation. 


\section{REFERENCES}

1. Waldmann V, Bougouin W, Karam N, et al.: Characteristics and clinical assessment of unexplained sudden cardiac arrest in the realworld setting: Focus on idiopathic ventricular fibrillation. Eur Heart ] 2018; 39:1981-1987.

2. Visser M, van der Heijden JF, Doevendans PA, Loh P, Wilde AA, Hassink RJ: Idiopathic Ventricular Fibrillation. Circ Arrhythmia Electrophysiol 2016; 9:e003817.

3. Haïssaguerre $M$, Shah $D C$, Jaïs $P$, et al.: Role of Purkinje conducting system in triggering of idiopathic ventricular fibrillation. Lancet 2002; 359:677-678.

4. Straus SMJM, Bleumink GS, Dieleman JP, Lei Van Der J, Stricker BHC, Sturkenboom MCJM: The incidence of sudden cardiac death in the general population. J Clin Epidemiol 2004; 57:98-102.

5. Chugh SS, Jui J, Gunson K, et al.: Current burden of sudden cardiac death: Multiple source surveillance versus retrospective death certificate-based review in a large U.S. community. J Am Coll Cardiol 2004; 44:1268-1275.

6. Priori SG, Wilde AA, Horie M, et al.: HRS/EHRA/APHRS Expert Consensus Statement on the Diagnosis and Management of Patients with Inherited Primary Arrhythmia Syndromes. Heart Rhythm 2013; 10:1932-1963.

7. Behr ER, Casey A, Sheppard M, Wright M, Bowker TJ, Davies MJ, McKenna WJ, Wood DA: Sudden arrhythmic death syndrome: a national survey of sudden unexplained cardiac death. Heart 2007; 93:601-605.

8. Krahn AD, Healey JS, Chauhan V, et al.: Systematic assessment of patients with unexplained cardiac arrest: Cardiac Arrest Survivors With Preserved Ejection Fraction Registry (CASPER). Circulation 2009; 120:278-285.

9. Visser M, van der Heijden JF, van der Smagt JJ, Doevendans PA, Wilde AA, Loh P, Hassink RJ: Long-Term Outcome of Patients Initially Diagnosed With Idiopathic Ventricular Fibrillation. Circ 
Arrhythmia Electrophysiol 2016; 9:e004258.

10. Ozaydin M, Moazzami K, Kalantarian S, Lee H, Mansour M, Ruskin JN: Long-Term Outcome of Patients With Idiopathic Ventricular Fibrillation: A Meta-Analysis. J Cardiovasc Electrophysiol 2015; 26:1095-1104.

11. Herman ARM, Cheung C, Gerull B, et al.: Outcome of Apparently Unexplained Cardiac Arrest: Results From Investigation and FollowUp of the Prospective Cardiac Arrest Survivors With Preserved Ejection Fraction Registry. Circ Arrhythm Electrophysiol 2016; 9:e003619.

12. Knecht S, Sacher F, Wright M, et al.: Long-Term Follow-Up of Idiopathic Ventricular Fibrillation Ablation. A Multicenter Study. J Am Coll Cardiol 2009; 54:522-528.

13. Champagne J, Geelen P, Philippon F, Brugada P: Recurrent cardiac events in patients with idiopathic ventricular fibrillation, excluding patients with the Brugada syndrome. BMC Med 2005; 3:1.

14. Crijns HJ, Wiesfeld AC, Posma JL, Lie KI: Favourable outcome in idiopathic ventricular fibrillation with treatment aimed at prevention of high sympathetic tone and suppression of inducible arrhythmias. Br Heart J 1995; 74:408-412.

15. Conte G, Caputo ML, Regoli F, Marcon S, Klersy C, Adjibodou B, Del Bufalo A, Moccetti T, Auricchio A: True idiopathic ventricular fibrillation in out-of-hospital cardiac arrest survivors in the Swiss Canton Ticino: prevalence, clinical features, and long-term followup. Europace 2017; 19:259-266.

16. Basso C, Aguilera B, Banner J, et al.: Guidelines for autopsy investigation of sudden cardiac death: 2017 update from the Association for European Cardiovascular Pathology. Virchows Arch 2017; 471:691-705.

17. Zipes DP, Wellens HJJ: Sudden Cardiac Death. Circulation 1998; 98:2334-2351.

18. Tan HL, Hofman N, Van Langen IM, Van Der Wal AC, Wilde AAM: Sudden unexplained death: Heritability and diagnostic yield of cardiological and genetic examination in surviving relatives. 
Circulation 2005; 112:207-213.

19. Siebermair J, Sinner M, Beckmann B-M, Laubender R, Martens E, Sattler S, Fichtner S, Estner H, Kaab S, Wakili R: Early repolarization pattern is the strongest predictor of arrhythmia recurrence in patients with idiopathic ventricular fibrillation: results from a single centre long-term follow-up over 20 years+. Europace 2016; 18:718-725.

20. Schwartz PJ, Ackerman MJ: The long QT syndrome: A transatlantic clinical approach to diagnosis and therapy. Eur Heart J 2013; 34:3109-3116.

21. Hayashi M, Denjoy I, Extramiana F, et al.: Incidence and risk factors of arrhythmic events in catecholaminergic polymorphic ventricular tachycardia. Circulation 2009; 119:2426-2434.

22. Antzelevitch C, Yan G-X: J-wave syndromes: Brugada and early repolarization syndromes. Heart Rhythm 2015; 12:1852-1866.

23. Corrado D, Wichter T, Link MS, et al.: Treatment of Arrhythmogenic Right Ventricular Cardiomyopathy/Dysplasia. Circulation 2015; 132:441-453.

24. O'Hanlon R, Mohiaddin RH: Inherited cardiomyopathies. Magn Reson Imaging Congenit Hear Dis 2012; 6966:211-229.

25. Behr ER, Dalageorgou C, Christiansen M, Syrris P, Hughes S, Tome Esteban MT, Rowland E, Jeffery S, McKenna WJ: Sudden arrhythmic death syndrome: Familial evaluation identifies inheritable heart disease in the majority of families. Eur Heart J 2008; 29:16701680.

26. Lahrouchi N, Raju H, Lodder EM, et al.: Utility of Post-Mortem Genetic Testing in Cases of Sudden Arrhythmic Death Syndrome. J Am Coll Cardiol 2017; 69:2134-2145.

27. Belhassen B, Viskin S, Fish R, Glick A, Setbon I, Eldar M: Effects of electrophysiologic-guided therapy with Class IA antiarrhythmic drugs on the long-term outcome of patients with idiopathic ventricular fibrillation with or without the Brugada syndrome. J Cardiovasc Electrophysiol 1999; 10:1301-1312.

28. Meissner MD, Lehmann MH, Steinman RT, Mosteller RD, Akhtar 
M, Calkins H, Cannom DS, Epstein AE, Fogoros RN, Liem LB:

Ventricular fibrillation in patients without significant structural heart disease: a multicenter experience with implantable cardioverterdefibrillator therapy. J Am Coll Cardiol 1993; 21:1406-1412.

29. Priori SG, Blomström-Lundqvist C, Mazzanti A, et al.: 2015 ESC Guidelines for the management of patients with ventricular arrhythmias and the prevention of sudden cardiac death. Eur Heart J 2015; 36:2793-2867.

30. Belhassen B, Viskin S, Fish R, Glick A, Setbon I, Eldar M: Effects of electrophysiologic-guided therapy with Class IA antiarrhythmic drugs on the long-term outcome of patients with idiopathic ventricular fibrillation with or without the Brugada syndrome. J Cardiovasc Electrophysiol 1999; 10:1301-1312.

31. Wilde AAM, Behr ER: Genetic testing for inherited cardiac disease. Nat Rev Cardiol 2013; 10:571-583.

32. Cirino AL, Harris S, Lakdawala NK, et al.: Role of Genetic Testing in Inherited Cardiovascular Disease. JAMA Cardiol 2017; 2:1153.

33. Kumar S, Peters S, Thompson T, Morgan N, Maccicoca I, Trainer A, Zentner D, Kalman JM, Winship I, Vohra JK: Familial cardiological and targeted genetic evaluation: low yield in sudden unexplained death and high yield in unexplained cardiac arrest syndromes. Heart Rhythm 2013; 10:1653-1660.

34. Steinberg C, Padfield GJ, Champagne J, et al.: Cardiac Abnormalities in First-Degree Relatives of Unexplained Cardiac Arrest Victims. Circ Arrhythmia Electrophysiol 2016; 9.

35. Honarbakhsh S, Srinivasan N, Kirkby C, Firman E, Tobin L, Finlay M, Hunter RJ, Murphy C, Lowe MD, Schilling RJ, Lambiase PD: Medium-term outcomes of idiopathic ventricular fibrillation survivors and family screening: a multicentre experience. Europace 2016;

36. Ramanathan C, Ghanem RN, Jia P, Ryu K, Rudy Y: Noninvasive electrocardiographic imaging for cardiac electrophysiology and arrhythmia. Nat Med 2004; 10:422-428.

37. Vijayakumar R, Silva JNA, Desouza KA, Abraham RL, Strom M, Sacher F, Van Hare GF, Haissaguerre M, Roden DM, Rudy Y: 
Electrophysiologic Substrate in Congenital Long QT Syndrome: Noninvasive Mapping With Electrocardiographic Imaging (ECGI). Circulation 2014; 130:1936-1943.

38. Nademanee $K$, Haissaguerre M, Hocini M, et al.: Mapping and Ablation of Ventricular Fibrillation Associated with Early Repolarization Syndrome. Circulation 2019; 1477-1490.

39. Postema PG, van Dessel PFHM, Kors JA, Linnenbank AC, van Herpen G, Ritsema van Eck HJ, van Geloven N, de Bakker JMT, Wilde AAM, Tan HL: Local Depolarization Abnormalities Are the Dominant Pathophysiologic Mechanism for Type 1 Electrocardiogram in Brugada Syndrome. A Study of Electrocardiograms, Vectorcardiograms, and Body Surface Potential Maps During Ajmaline Provocation. J Am Coll Cardiol 2010; 55:789-797.

40. Zhang J, Sacher F, Hoffmayer K, et al.: Cardiac Electrophysiological Substrate Underlying the ECG Phenotype and Electrogram Abnormalities in Brugada Syndrome Patients. Circulation 2015; 131:1950-1959.

41. Castro Hevia J, Antzelevitch C, Tornés Bárzaga F, Dorantes Sánchez M, Dorticós Balea F, Zayas Molina R, Quiñones Pérez MA, Fayad Rodríguez Y: Tpeak-Tend and Tpeak-Tend Dispersion as Risk Factors for Ventricular Tachycardia/Ventricular Fibrillation in Patients With the Brugada Syndrome. J Am Coll Cardiol 2006; 47:1828-1834.

42. Lubinski A, Kornacewicz-Jach Z, Wnuk-Wojnar AM, Adamus J, M. K, Królak T, Lewicka-Nowak E, Radomski M, Swiatecka G: The Terminal Portion of the T Wave: A New Electrocardiographic Marker of Risk of Ventricular Arrhythmias. Pacing Clin Electrophysiol 2000; 23:1957-1959.

43. Verrier RL, Klingenheben T, Malik M, El-Sherif N, Exner D V., Hohnloser SH, Ikeda T, Martínez JP, Narayan SM, Nieminen T, Rosenbaum DS: Microvolt T-wave alternans: Physiological basis, methods of measurement, and clinical utilityconsensus guideline by international society for Holter and noninvasive Electrocardiology. J Am Coll Cardiol 2011; 58:1309-1324.

44. Gulshan V, Peng L, Coram M, et al.: Development and validation of 
a deep learning algorithm for detection of diabetic retinopathy in retinal fundus photographs. JAMA 2016; 316:2402-2410.

45. Attia ZI, Noseworthy PA, Lopez-Jimenez F, Asirvatham SJ, Deshmukh AJ, Gersh BJ, Carter RE, Yao X, Rabinstein AA, Erickson BJ, Kapa S, Friedman PA: An artificial intelligence-enabled ECG algorithm for the identification of patients with atrial fibrillation during sinus rhythm: a retrospective analysis of outcome prediction. Lancet 2019; 394:861-867.

46. Li Fei-Fei, Fergus R, Perona P: One-shot learning of object categories. IEEE Trans Pattern Anal Mach Intell 2006; 28:594-611.

47. Pan SJ, Yang Q: A Survey on Transfer Learning. IEEE Trans Knowl Data Eng 2010; 22:1345-1359.

48. Selvaraju RR, Cogswell M, Das A, Vedantam R, Parikh D, Batra D: Grad-CAM: Visual Explanations from Deep Networks via GradientBased Localization. 2017 IEEE Int Conf Comput Vis 2017, pp. 618-626.

49. Haissaguerre $M$, Shoda $M$, Jais $P$, et al.: Mapping and ablation of idiopathic ventricular fibrillation. Circulation 2002; 106:962-967.

50. Oostendorp TF, van Dessel PFHM, Coronel R, Belterman C, Linnenban AC, van Schie IH, van Oosterom A, Oosterhoff $P$, van Dam PM, de Bakker JMT: Noninvasive detection of epicardial and endocardial activity of the heart. Netherlands Heart J 2011; 19:488-491.

51. Duchateau J, Sacher F, Pambrun T, Derval N, Chamorro-Servent J, Denis A, Ploux S, Hocini M, Jaïs P, Bernus O, Haïssaguerre M, Dubois R: Performance and limitations of noninvasive cardiac activation mapping. Heart Rhythm 2019; 16:435-442.

52. Orini $M$, Taggart $P$, Lambiase PD: In vivo human sock-mapping validation of a simple model that explains unipolar electrogram morphology in relation to conduction-repolarization dynamics. J Cardiovasc Electrophysiol 2018; 29:990-997.

53. Alders M, Koopmann TT, Christiaans I, et al.: Haplotype-Sharing Analysis Implicates Chromosome 7q36 Harboring DPP6 in Familial Idiopathic Ventricular Fibrillation. Am J Hum Genet 2009; 84:468- 
476.

54. Marsman RF, Barc J, Beekman L, Alders M, Dooijes D, Van Den Wijngaard A, Ratbi I, Sefiani A, Bhuiyan ZA, Wilde AAM, Bezzina CR: A mutation in CALM1 encoding calmodulin in familial idiopathic ventricular fibrillation in childhood and adolescence. J Am Coll Cardiol 2014; 63:259-266.

55. Boyle EA, Li YI, Pritchard JK: An Expanded View of Complex Traits: From Polygenic to Omnigenic. Cell 2017; 169:1177-1186.

56. Wray NR, Goddard ME, Visscher PM: Prediction of individual genetic risk of complex disease. Curr Opin Genet Dev 2008; 18:257-263.

57. Rumsfeld JS, Joynt KE, Maddox TM: Big data analytics to improve cardiovascular care: Promise and challenges. Nat Rev Cardiol 2016; 13:350-359. 


\section{Nederlandse Samenvatting}

Na plotselinge hartstilstand is het vinden van een onderliggende oorzaak van belang. Het uitsluiten van alle gangbare oorzaken, zoals onderliggende cardiomyopathie of electrische hartziekten, vereist uitgebreide diagnostiek en is noodzakelijk om de diagnose idiopathisch ventrikelfibrilleren (IVF) te kunnen stellen. Deze zeldzame patientengroep met een ogenschijnlijk structureel en electrisch normaal hart heeft baat bij de ontwikkeling van nieuwe diagnostische mogelijkheden en uitbreiding van kennis over het onderliggend substraat en mechanisme van hun hartritmestoornissen om de prognose en behandeling van het ziektebeeld te verbeteren.

In hoofdstuk 2 wordt een overzicht gegeven van idiopathisch ventrikelfibrilleren. We bespreken het belang van verschillende onderzoeken die moeten worden verricht om andere oorzaken uit te sluiten en geven een overzicht van de prognose en behandeling. Daarnaast blikken we vooruit op de ontwikkelingen en bespreken de lopende studies naar idiopathisch ventrikelfibrilleren in Nederland.

In hoofdstuk 3 wordt de relevantie van diagnostische tests bij jonge slachtoffers van plotselinge hartdood en het cardiogenetisch onderzoek bij familieleden besproken. Het opsporen van een erfelijke aandoening en het verrichten van familieonderzoek vereist nauwkeurig en uitgebreid onderzoek bij de overledene. Bij dit proces zijn meerdere disciplines betrokken en samenwerking tussen de verschillende specialismen vergroot de kans op het opsporen van een erfelijke hartaandoening. We bespreken de mogelijkheden die er zijn voor onderzoek bij de overledene en familieleden in verschillende scenarios en hun bijdrage aan het vinden van een erfelijke hartaandoening in de familie.

In hoofdstuk 4 worden de uitkomsten van ICD therapie bij 217 patienten met idiopathisch ventrikelfibrilleren beschreven. In deze groep kwam bij 66 (30\%) patienten een recidief ventriculaire ritmestoornis 
voor waarvoor ICD-therapie nodig was tijdens een mediane follow-up periode van 6.1 jaar. Daarnaast kregen 38 (17.5\%) patienten onterechte ICD therapie en kwamen bij 32 (14.7\%) patienten ICD-gerelateerde complicaties voor. Symptomen voorafgaand aan de hartstilstand, tekenen van geleidingsziekte en dragerschap van het DPP6 risico haplotype waren onafhankelijke voorspellers voor terechte ICD shocks.

In hoofstuk 5 beschrijven we de uitkomsten van cardiogenetisch onderzoek bij familieleden van patienten met idiopathisch ventrikelfibrilleren. In totaal zijn 189 familieleden uit 89 families onderzocht die minstens een 12-kanaals rust-ECG hadden gehad. Bij $114(79 \%)$ famlieleden was ook een echocardiogram verricht en 170 (90\%) familieleden hadden meerdere onderzoeken ondergaan. Bij 5 (3\%) familieleden uit 4 (4\%) families werd een erfelijke hartziekte gevonden. Twee familieleden bleken het DPP6-risico haplotype te dragen dat ook bij de index patient was geïdentificeerd. In drie afzonderlijke families werd bij een van de ouders van de index patient het Brugadasyndroom vastgesteld na een positieve provocatietest met ajmaline. Deze bevindingen geven aan dat de opbrengst van familieonderzoek bij idiopathisch ventrikelfibrilleren lager ligt dan verwacht.

In hoofdstuk $\mathbf{6}$ bestuderen we het gebruik van nieuwe repolarisatieparameters bij 'electrocardiografic imaging' (ECGI). Om de repolarisatie van het hart te beschrijven wordt op dit moment voornamelijk gebruik gemaakt van repolarisatie tijd, maar niet van andere parameters die afhankelijk zijn van morphologie en hartslagvariatie en gebruikt worden voor risicostratificatie voor hartritmestoornissen op het 12-kanaals ECG. We vonden dat het meten van deze nieuwe repolarisatieparameters in de gereconstrueerde elektrogrammen van ECGI mogelijk is, op een volledig geautomatiseerde manier kan worden gedaan en kan helpen bij het beschrijven van het onderliggende aritmogene substraat. Ook vonden wij dat steile repolarisatietijdgradiënten konden worden gevonden in regio's op het hart met vlakke repolarisatie op het lokale elektrogram. 
Hoofdstuk 7 geeft een beschrijving van een diep neuraal netwerk (DNN) voor de classificatie van 12-afleidingen ECGs in vier triage-categorieën: normaal, abnormaal-niet acuut, subacuut en acuut. We ontwikkelden een 37-laags convolutioneel residueel diep neuraal netwerk wat getrained werd op een dataset van 336.835 ECG opnames van 142.040 patienten geannoteerd door een individuele arts en wat gevalideerd werd op een consensus validatieset ( $n=984$ ), die door een panel van vijf cardioloogelectrophysiologen beoordeeld was. Het neurale netwerk kon uitstekend discrimineren tussen de vier categorieen (C statistiek van 0,95 (95\% BI 0,94 - 0,96)) en presteerde daarmee beter dan de individuele artsassistent en het conventionele computeralgoritme. Hiermee hebben we aangetoond dat een diep neuraal netwerk nauwkeurig kan worden gebruikt voor 12-kanaals ECG interpretatie.

In hoofdstuk 8 beschrijven we het belang van diagnostiek bij idiopathisch ventrikelfibrilleren aan de hand van enkele casus van patienten met initieel onverklaard ventrikelfibrilleren (VF) die later tijdens de follow-up periode gediagnosticeerd worden met arrhythmogene cardiomyopathie. VF kan de eerste klinische manifestatie van erfelijke ziekte zijn en wij zagen dat het vinden van een diagnose in een later stadium verklaard kan worden door verbetering van diagnostische hulpmiddelen, ziekteprogressie of verkeerde interpretatie van de aanvankelijk beschikbare gegevens. Ondanks dat het vinden van een diagnose bij de index patient niet altijd leidt tot een verandering van beleid, kan bij familieleden een juiste diagnose leiden tot cascadescreening als een eerste stap naar risicostratificatie. Om aangedane familieleden op te sporen vóór het optreden van ventriculaire aritmieën en plotse dood, is het belangrijk om patienten met idiopathisch ventrikelfibrilleren periodiek te herevalueren.

\section{Conclusies}

In dit proefschrift zijn de ontwikkelingen op het gebied van diagnostiek en behandeling van idiopathisch ventrikelfibrilleren beschreven. Het belang 
van uitgebreide diagnostiek om een onderliggende oorzaak aan te tonen dan wel uit te sluiten heeft belangrijke consequenties voor het beleid van zowel de index patient als van de familieleden. Het inschatten van het risico op levensbedreigende hartritmestoornissen en het karakteriseren van onderliggend substraat in deze patientengroep behoeft meer onderzoek naar diagnostische en therapeutische mogelijkheden. 
Dankwoord

Prof. dr. Doevendans, beste Pieter, bedankt voor uw steun en het vertrouwen tijdens mijn promotietraject. Bij mijn sollicitatie kreeg ik de vraag, wat vind je interessant binnen de cardiologie? "Ritmestoornissen" antwoordde ik. "Dan moet je met Rutger Hassink gaan praten." En zo geschiedde. Een keuze waar ik tot de dag van vandaag heel blij mee ben. Dank voor de gesprekken die we afgelopen jaren hebben gehad, die hielpen om met een frisse blik te blijven kijken en nieuwe ideeën op te doen.

Dr. Hassink, beste Rutger, een begeleider als jij kan ik iedereen toewensen. Altijd to-the-point, maar ook met humor en betrokken wanneer ik dat nodig had. We hebben in de afgelopen jaren veel studies en samenwerkingen opgezet en daarbij was jouw praktische inslag onontbeerlijk. Al beweer je zelf af en toe dat je niet heel efficiënt bent, je hebt ondanks alle drukte altijd tijd gehad voor overleg. Bedankt voor alle hulp de afgelopen jaren.

Alle coauteurs dank voor jullie bijdragen en commentaren die hebben geholpen bij het tot stand komen van dit proefschrift.

Dank aan alle collega's van de elektrofysiologie: Jeroen van der Heijden, Peter Loh, Matthias Meine, Nick Clappers, Anton Tuinenburg, Moniek Cox, Irene Hof, Samir Brka en Niels Jongejan. Bedankt voor alle hulp en meedenken bij het opzetten van de onderzoeken met de idiopathisch VF patiënten. Ik heb erg veel gehad aan de onderwijsmomenten en genoten van de humor op de vroege vrijdagochtend!

Ik wil de leden van de beoordelingscommissie, prof. dr. Volders, prof. dr. Wilde, prof. dr. Schalij, prof. dr. van den Berg en prof. dr. van Tintelen bedanken voor het kritisch lezen en beoordelen van mijn proefschrift.

Dr. Baas, beste Annette, bedankt voor je hulp bij al het genetica en fam- 
ilie onderzoek wat bij idiopathisch VF en plotse dood komt kijken. Jouw inbreng heeft erg geholpen om ook de interessante kanten van de genetica te zien.

Prof. dr. Folkert Asselbergs, Joyce en Katrien. Met jullie samen heb ik ook de stap van cardiologie naar global health kunnen nemen, wat zeker van pas gaat komen straks in Malawi. Jullie enthousiasme en doorzettingsvermogen bij ons Learning Healthcare project heeft ervoor gezorgd dat dit ambitieuze idee een goed uitgewerkt projectvoorstel is geworden en ik weet zeker dat dit nog een mooi vervolg gaat krijgen!

Dames van de R\&D, bedankt voor de gezelligheid op de gang, de Q5bakt/borrels en dat ik altijd bij jullie terecht kon voor praktische vragen of gewoon een praatje.

Alle leden van de staf Cardiologie, hartelijk dank voor de samenwerking! Ook wil ik graag alle laboranten van de hartfunctie en Ronald Groenemeijer bedanken voor de ondersteuning.

Mijn kamergenootjes wil ik bedanken: Evangeline, ik ken niemand die zo veel tegelijk doet en ook nog eens goed borrels en weekendjes kan organiseren. Ik ga je verhalen over wat je nu weer dit weekend hebt meegemaakt (hersenschudding, huis verbouwd, duim afgesneden) missen! Timion, als hardwerkende onderzoeker en top tennisser weet je gelukkig goed waar je talenten liggen, want lasergamen en tafelvoetballen gaat je toch een stuk minder goed af! Astrid, het was altijd leuk met jou op de kamer en ik ben blij dat je weer terug bent. Rosanne, succes met de weg vinden in het UMC. Bart, Sophie, Loek, bedankt voor de gezelligheid!

Dan mijn mede-onderzoekers bij de cardiologie: Rik, toch wel een beetje de verbindende factor in $\mathrm{Q}$, veel succes bij de radiologie! Nynke, wij waren ongeveer gelijk begonnen dus we konden gelukkig veel tips uitwisselen, en nu (bijna) gelijk promoveren, veel succes! Aernoud en Max, professoren-in-wording? Oreren kunnen jullie gelukkig al, bedankt voor 
de mooie discussies. Odette en Steven, allemaal begonnen in het Gelre en hier bij de EFO terecht gekomen, ik kon altijd lachen om jullie droge humor. Succes met de opleiding! René, we zijn samen toch een heel eind gekomen met het automatiseren van een ECG. Ik hoop dat het je met Rutger en 'the Beast', tussen het darten door, gaat lukken! Arjan, Deep Learning Bingo blijft toch wel een van de beste AI uitvindingen van de afgelopen jaren. Marijn, Phillipe, Thijs, Machteld, Mimount, Mark, Marijke, Janine, Laurens, Karim, David, Agnieska, Mirthe, Wouter, Nicole, bedankt voor alle gezellige momenten, borrels en weekendjes!

Alle (TG-)studenten die ik heb mogen begeleiden: Babette, Huub, Laurien, Esmée, Bernice en Diantha, bedankt voor jullie inzet en de mooie projecten die jullie hebben afgeleverd.

Mijn vrienden uit Amsterdam, Ilse, Kristianne, Ruth, Tessa, Evelyn, Alisa, Jordi, Daniël, Niels heel erg bedankt voor alle steun en interesse de afgelopen jaren. Ondanks dat ik steeds verder weg ging wonen en we elkaar wat minder gezien hebben zijn de etentjes en feestjes altijd een mooie gelegenheid geweest om er weer ouderwets een mooie avond van te maken!

Ook Frank, Jeff, Nina, Marije, Chase, bedankt voor jullie gezelligheid en gastvrijheid de afgelopen jaren.

Mijn paranimfen: Rob, blij dat je ook net als ik vanuit het verre oosten de stap naar Utrecht hebt gemaakt. We konden het altijd heel goed samen vinden, weten alle ins en outs van een ajmalinetest en hebben veel gelachen om de eigenaardigheden van de EFO. Bedankt voor alle mooie momenten van de afgelopen jaren en succes met de laatste loodjes! Sanne, mijn opvolgster. Ik ben heel blij dat ik afgelopen jaar een sparringspartner had om alles mee te kunnen delen. Je bent top in je werk, maar ik ga ook vooral onze koffie- en op het laatste moment langs de Mac gaan omdat we veel te laat bij de refereeravond aankwamen momentjes missen. 
Lieve Familie, pap, mam, Laura, Sander, Jan, Jet, Feijo, we hebben veel meegemaakt met elkaar de afgelopen jaren waardoor we ook veel dichter bij elkaar zijn gekomen en ik jullie gelukkig allemaal familie mag noemen. Ik heb veel gehad aan de steun en vertrouwen die ik gekregen heb van jullie tijdens mijn hele promotietijd, bedankt daarvoor. Jet, je hebt altijd een speciaal plekje in mijn hart.

Lieve Bente, met jou kan ik de hele wereld aan. We hebben elkaar de afgelopen jaren overal in gesteund en een hoop mooie stappen gemaakt. Ons volgende avontuur in Malawi is iets waar ik heel trots op ben dat het ons gelukt is en heel blij ben dat ik het met jou aan kan gaan. 


\section{Curriculum Vitae}

Lennart Johannes Blom was born on the 27th of November, 1990 in Bergen op Zoom, The Netherlands. He attended secondary education at the Stedelijk Gymnasium Leiden, from which he graduated in 2008. The same year he started his medical training at the VU Amsterdam. He received his Bachelor degree in Medicine in 2011. After his Bachelor he proceeded to study a Minor Development Studies and went to Shanghai University, China for an International Business Course. In 2012 he started the Master program of Medicine, during which he obtained an interest in Cardiology which resulted in a research internship at the Cardiology department of the MUMC+ in Maastricht.

After obtaining his Medical degree in 2015, he worked as a resident at the Cardiology department of the Gelre Ziekenhuis in Apeldoorn. In Februari 2017 he started as a PhD candidate at the department of Cardiology of the University Medical Center Utrecht under supervision of Prof.dr. Doevendans and dr. Hassink. The results of his PhD research are presented in this thesis. He will defend his thesis entitled "Progress in diagnosis and management of idiopathic ventricular fibrillation" on the 14th of April 2020. In 2020 he will start working at the Mangochi District Hospital, Mangochi, Malawi as a medical doctor/researcher with a focus on non-communicable diseases. 
